\title{
Development and validation of a novel counter-immunoelectrophoresis assay for the detection of antibodies against extractable nuclear antigens
}

By

Reuben Wallis

A thesis submitted to the Victoria University of Wellington in fulfillment of the requirements for the degree of Master of Molecular Bioscience

Victoria University of Wellington 2012 



\section{Acknowledgements}

First and foremost I would like to acknowledge and thank my supervisors, Dr. Neil Cook, and Dr. Bill Jordan. Their help and guidance have been indispensable over the course of this project.

I would also like to thank Dr. Kylie Hood (Arotec Diagnostics, Wellington) for all her advice, and help editing my thesis. I am grateful for the financial assistance provided by Arotec Diagnostics, without it I would never have been able to complete my thesis. To all the staff at Arotec Diagnostics you are great to work with and I appreciate the support you have given me.

I would like to thank the Wellington Hospital Immunology Department and Dr. Richard Steele for providing the patient serum samples, and in particular Glennis White who accommodated my intrusions into her laboratory. 


\section{Abstract}

The identification of autoantibodies is a primary diagnostic marker for the diagnosis of some autoimmune diseases. The sera of patients with connective tissue diseases commonly contain autoantibodies that target nuclear antigens. As such these antibodies are called antinuclear antibodies (ANAs). Furthermore, the clinical identification of ANAs in patient sera to specific nuclear antigens is a primary tool for the diagnosis of connective tissue diseases. For this purpose specific extractable nuclear antigens (ENAs) are used. The most commonly employed laboratory technique for the detection of ENA antibodies is the enzyme linked immunoabsorbant assay (ELISA). ELISA is a simple technique that can be automated and provides high diagnostic sensitivity for the detection of ENA antibodies comparatively to a counter immunoelectrophoresis (CIE) assay. However, compared to CIE ELISA lacks diagnostic specificity. Therefore there is a demand for an assay with high diagnostic specificity as a secondary diagnostic test for the detection of ENA antibodies. The central aim of this project was to develop and validate a novel CIE assay that used fluorescently labelled ENAs to detect ENA antibodies in patient serum.

Development of the assay began with comparative testing of fluorescently labelled and unlabelled antigens to confirm that the immunochemical properties of the antigen remained intact. The CIE assay conditions were optimised for the detection of four ANAs SSA, SSB, RNP/Sm, and Sm using their corresponding ENAs. Assay conditions optimised were flurophore type, gel composition, buffer composition, antigen concentration, the running time, and analytical specificity.

Evaluation of 281 clinical sera samples known to have previously tested positive test by ANA indirect immunofluorescence were performed using the optimised CIE assay and ELISA. The inter-rater agreement between the CIE assay and ELISA results was determined. Clinical details were used to retrospectively classify the clinical sera samples into clinical categories and case groups. This data was used for the diagnostic comparison of the CIE assay and ELISA results. 
There was strong inter-rater agreement between the SSA CIE assay and ELISA results. The diagnostic specificity and positive likelihood ratio values of the SSA CIE assay were superior to those of the ELISA, while diagnostic sensitivity and the negative likelihood ratio values were approximately the same. There was strong interrater agreement between the RNP/Sm CIE assay and ELISA. However, it was observed that the statistical measures of assay accuracy (diagnostic specificity and sensitivity, positive likelihood ratio, and negative likelihood ratio) for the RNP/Sm ELISA were superior to those of CIE assay. The diagnostic specificity of the SSB and $\mathrm{Sm}$ CIE assays were higher than that of their respective ELISAs. This was also true for the positive likelihood ratio values of the SSB and Sm CIE assays when compared to their respect ELISAs.

The SSA CIE assay results suggest that this assay maybe a suitable replacement for ELISA as a diagnostic tool for the identification of anti-SSA antibodies and Sjogren's syndrome. The SSB and Sm CIE assay results suggest that these assays would be suitable as secondary confirmatory diagnostic assays for the identification of their respective ENA antibodies. However, further performance evaluation of the assays within a routine diagnostic laboratory is required. 


\section{Abbreviations}

$A b$

AF488

$\mathrm{Ag}$

ANA

APC

BCR

BSA

CIE

CDR

CTD

DC

D488

DID

ENA

ELISA

$\mathrm{Fab}$

Fc

IB

$\lg$

IIF

IL

L.E. cell

LIA

LR

$\mathrm{MHC}$

$\mathrm{NHS}$

NLR

NPV

PBST

PM/DM

PLR antibody

Alexafluor 488

antigen

antinuclear antibody

antigen presenting cell

B-cell receptor

bovine serum albumin

counter immunoelectrophoresis

complement determining region

connective tissue disease

dendritic cells

Dylight 488

double immunodiffusion

extractable nuclear antigen

enzyme linked immunoabsorbant assay

fragments of antigen binding

fragment crystalisable

immunoblotting

immunoglobulin

indirect immunofluorescence

interleukin

lupus erythematous cell

line immunoassay

likelihood ratio

major histocompatibility complex

$\mathrm{N}$-hydroxysuccinimide

negative likelihood ratio

negative predictive value

phosphate buffered saline solution with tween 20

polymyositis/dermatomyositis

positive likelihood ratio 
PPV

PRR

RNA pol III

SDS-PAGE

snRNP

SLE

SS

SSc

TCR

$\mathrm{T}_{\mathrm{H}}$

$\mathrm{T}_{\text {reg }}$

TSH-R positive predictive value

pattern recognition receptors

RNA polymerase III

sodium dodecyl sulphate polyacrylamide gel electrophoresis

small nuclear ribonucleoprotein

systemic lupus erythematosus

Sjogrens syndrome

systemic sclerosis

T-cell receptor

T-helper cell

T-regulatory cell

thyroid-stimulating hormone receptor 


\section{Table of Contents}

Development and validation of a novel counter-immunoelectrophoresis assay for the detection of ENA antibodies

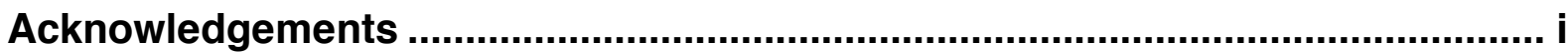

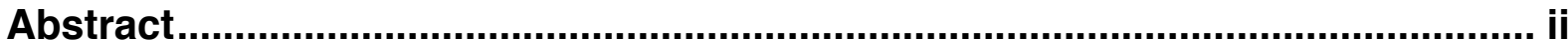

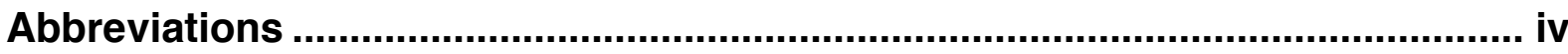

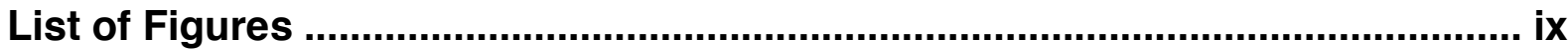

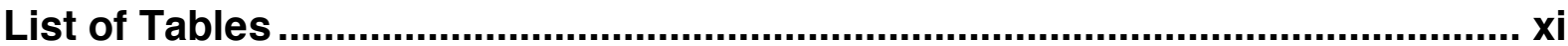

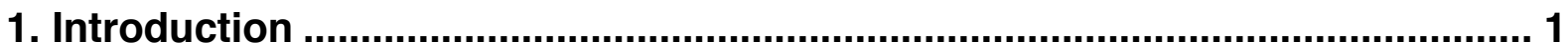

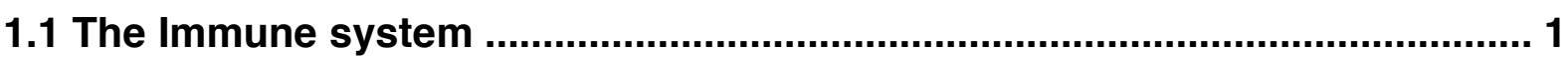

1.1.1 Antigens ........................................................................................................

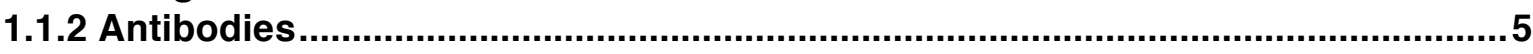

1.1.3 Antigen-Antibody Interactions.......................................................................

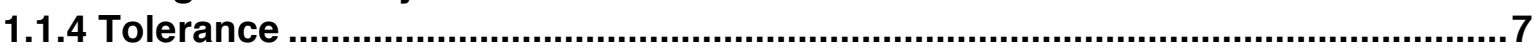

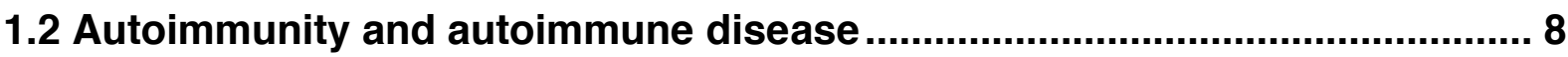

1.2.1 Loss of tolerance and autoimmunity .................................................................

1.2.2 Autoimmune diseases.........................................................................................10

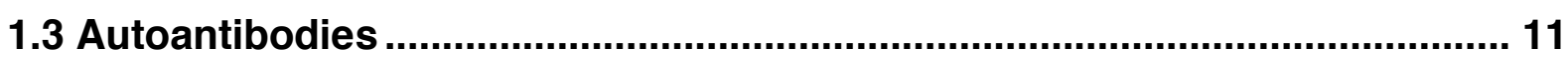

1.4 Antinuclear antigen antibody testing......................................................... 13

1.4.1 Whole cell screening of ANAs .....................................................................13

1.4.2 Detection of autoantibodies to extractable nuclear antigens ............................14

1.5 Comparison of diagnostic screening methods........................................... 15

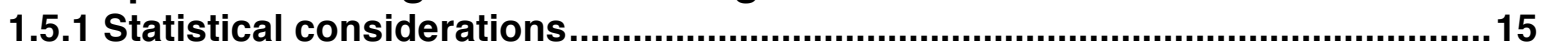

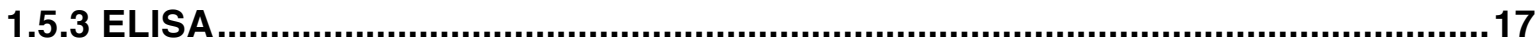

1.5.4 Immunoblotting ……........................................................................................17

1.5.5 RNA precipitation .......................................................................................18

1.5.6 Immunoprecipitation; DID and CIE ............................................................18

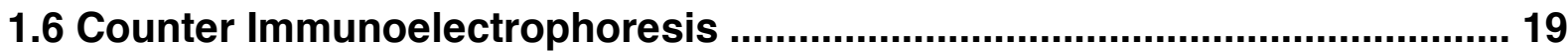

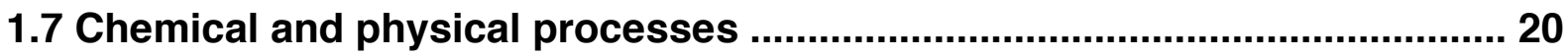

1.7.1 Precipitation reactions ……............................................................................20

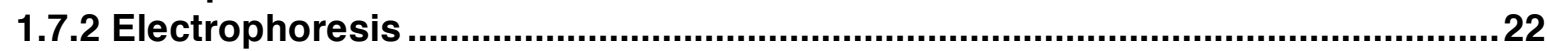

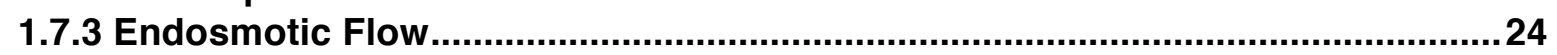

1.7.4 Immunoelectrophoresis .................................................................................25

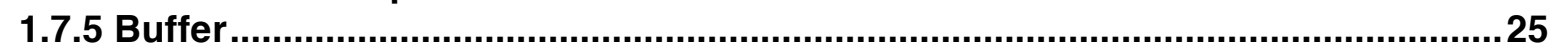

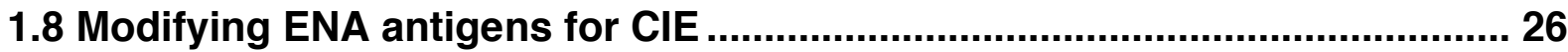

1.8.1 Modification of ENA antigens with Alexa Fluor ${ }^{\circledR} 488$ amine reactive dye ........27

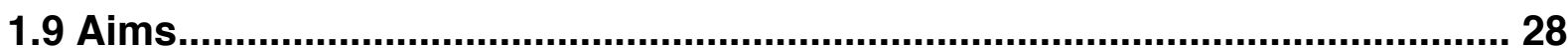

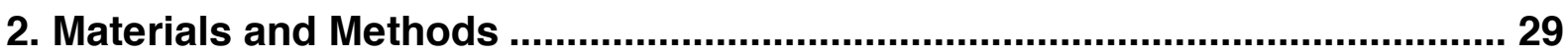

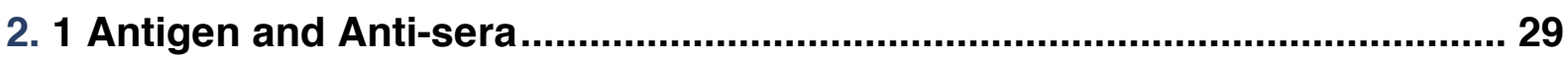

2.2 Preparation and Labelling of Protein with Dylight-488 Fluor....................... 29 
2.3 Antigenicity of Labelled vs Unlabelled Proteins........................................ 29

2.4 Fluorecent Intensity of Labelled Protein ........................................................... 31

2.5 CIE Apparatus modifications and configurations....................................... 32

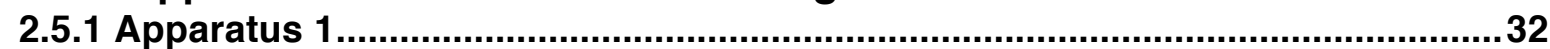

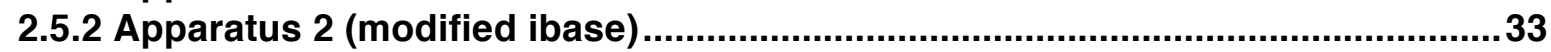

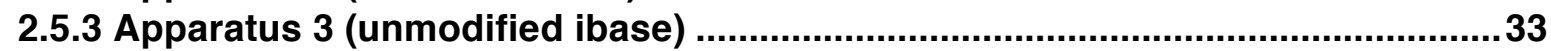

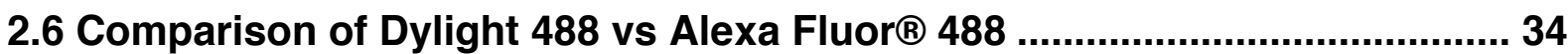

2.6.1 Preparation and labelling of protein with Alexa Fluor ${ }^{\circledR} 488 \ldots \ldots \ldots \ldots \ldots \ldots . . . . . . . . . . . .34$

2.6.2 Comparison of fluorescent intensity D488 vs AF488 .......................................34

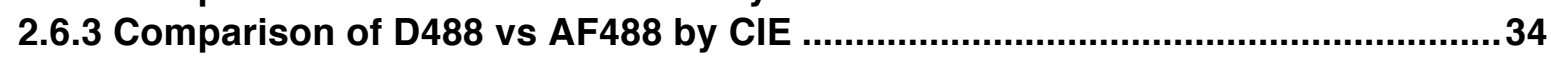

2.7 Optimisation of Counter Immunoelectrophoresis .................................... 35

2.7.1 Standard Counter Immunoelectrophoresis Method ........................................35

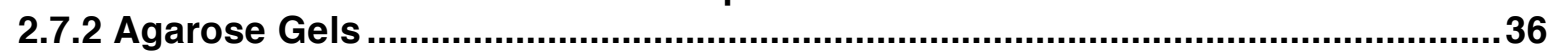

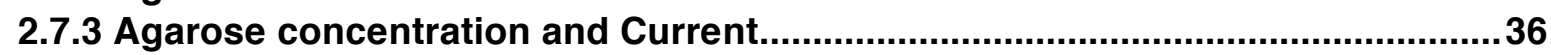

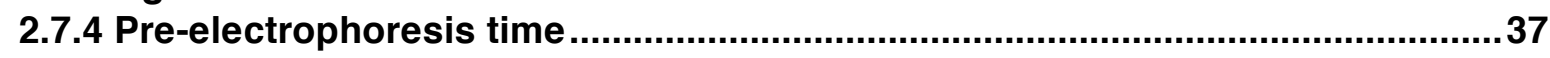

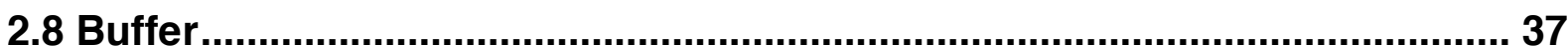

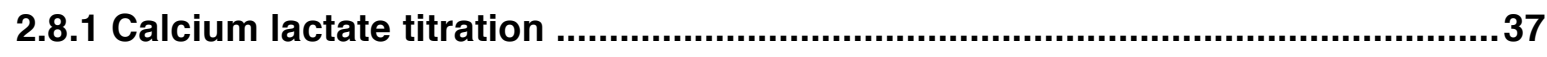

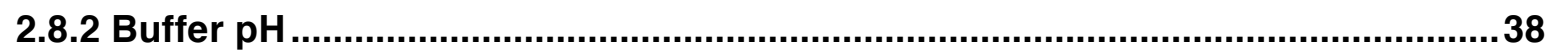

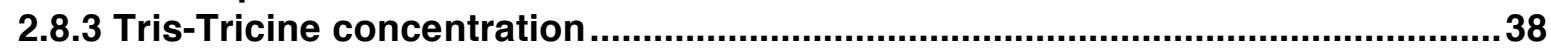

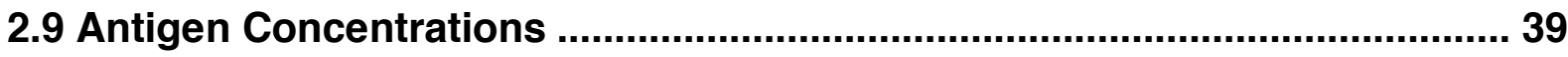

2.10 Antigen Concentration and Analytical Sensitivity ......................................... 39

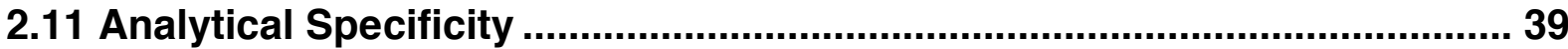

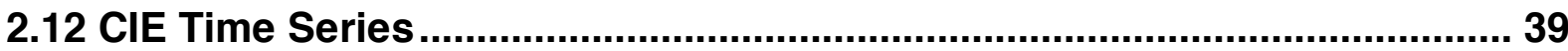

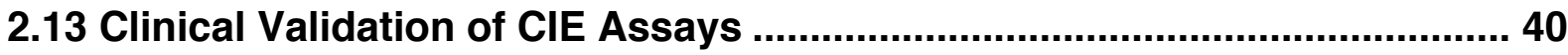

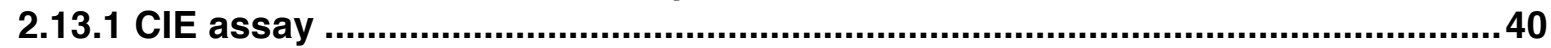

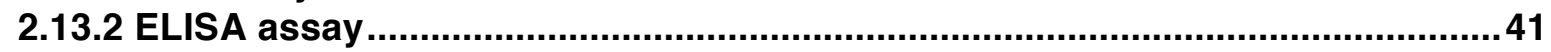

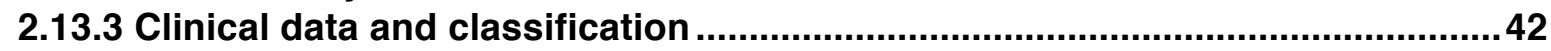

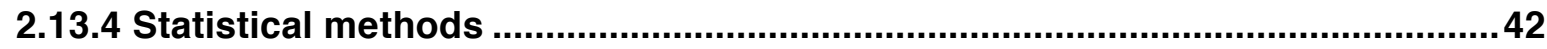

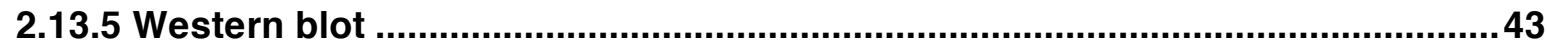

2.14 Double Immunodiffusion .......................................................................... 43

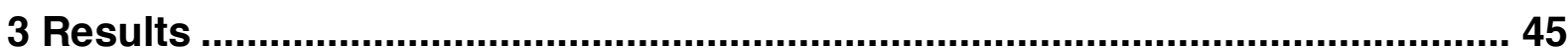

3.1 Preparation and Labelling of Protein with Dylight 488 Fluor....................... 45

3.2 Antigenicity of Labelled vs Unlabelled Protein..................................................46

3.3 Fluorescent Intensity of D488-Labelled Proteins ....................................... 50

3.4 Comparison of D488 and AF488 .............................................................. 52

3.4.1 Comparison of the relative fluorescent intensity of D488 and AF488 labelled

ENAs.

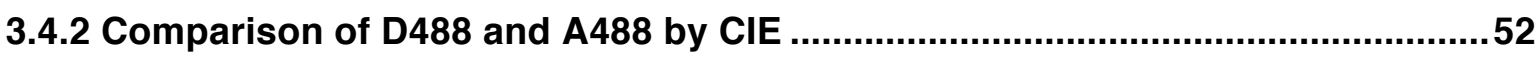

3.5 Optimisation of Counter Immunoelectrophoresis ...................................... 56

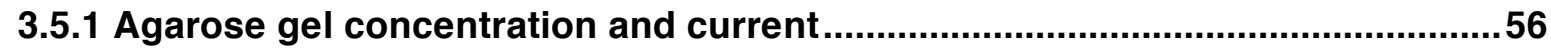

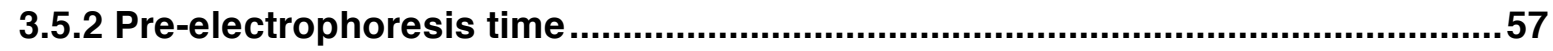

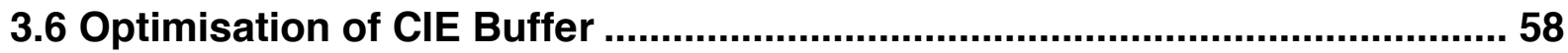

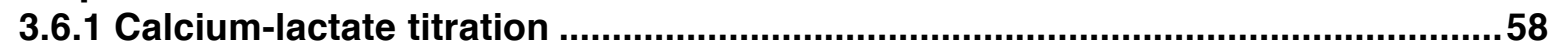

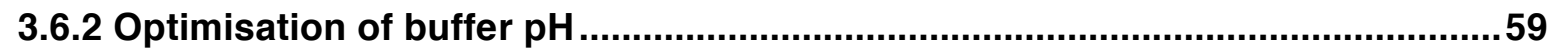

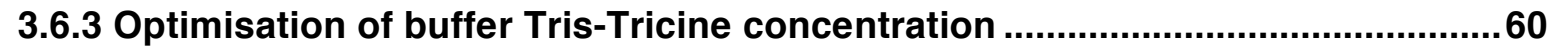




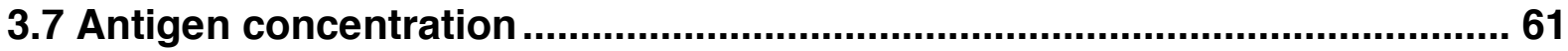

3.7.1 Antigen titration...................................................................................61

3.7.2 Antigen concentration vs sera titration series................................................62

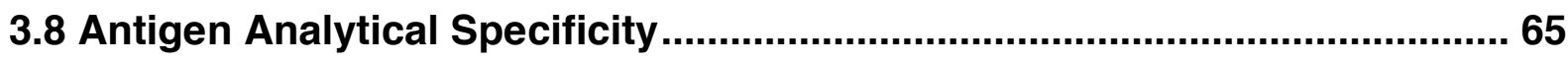

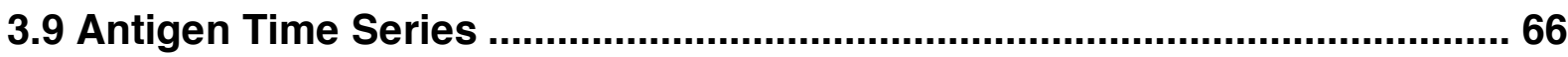

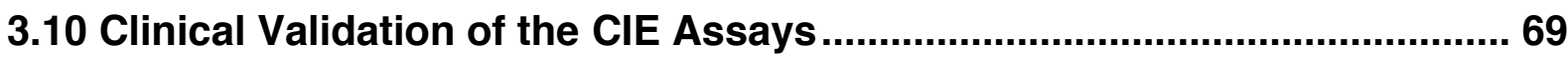

3.10.1 Correlation between CIE and ELISA clinical samples scores .......................69

3.10.2 Correlation between clinical data and ELISA and CIE diagnostic assays ......71

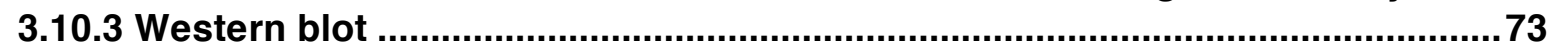

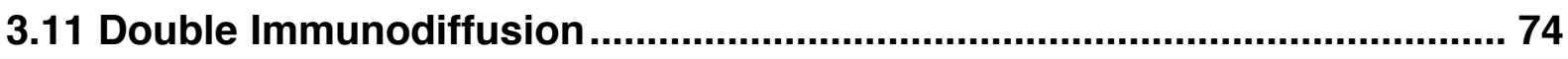

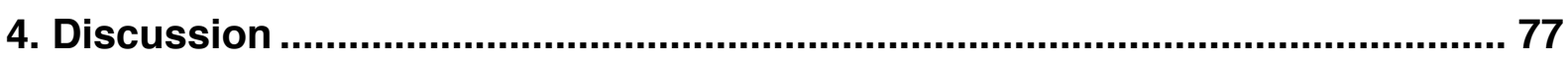

4.1 Labelling and comparison of D488 vs AF488 ...............................................77

4.1 Optimisation of the ENA CIE assays .................................................................

4.2 Clinical Evaluation of the ENA CIE assays ..........................................................8

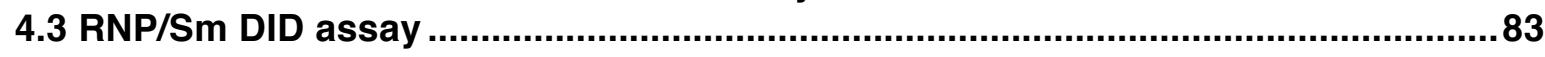

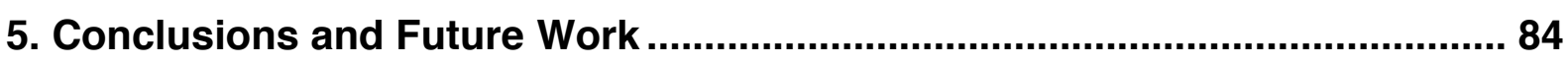

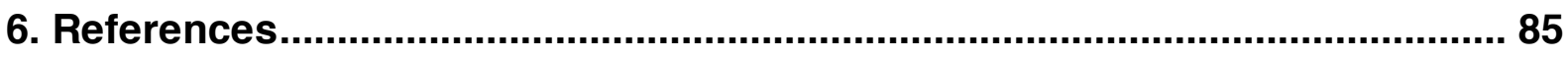

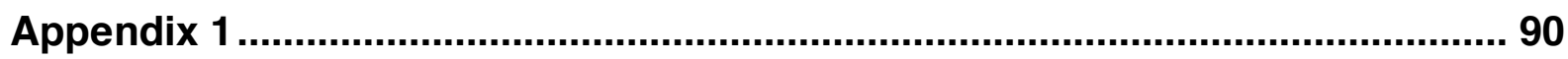




\section{List of Figures}

Figure 1 Diagrammatic representation of antigen and antibody migration

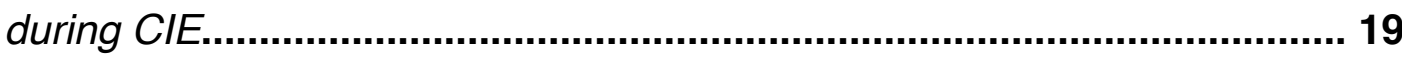

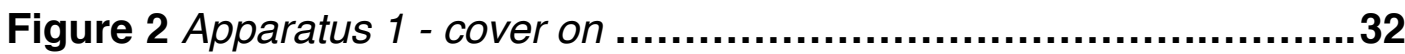

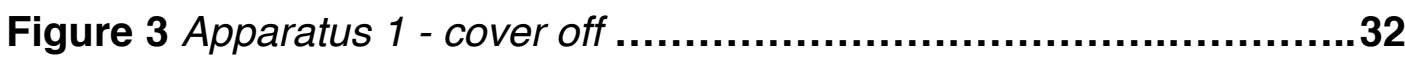

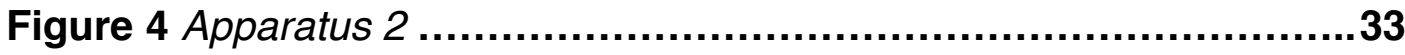

Figure 5 Apparatus 3 inside Gel Doc XR ......................................... 33

Figure 6 CIE Apparatus 2 cover off - Wick and gel placement ................ 35

Figure 7 CIE Apparatus 2 cover on - assay running .............................36

Figure 8 Dylight 488 labelling of ENA proteins reaction time .................... 45

Figure 9 Comparison of antigen labelling ...............................................47

Figure 10 Comparison of labelled \& unlabelled SSA antigen by ELISA ... 48

Figure 11 Comparison of labelled \& unlabelled SSB antigen by ELISA ... 48

Figure 12 Comparison of labelled \& unlabelled RNP/Sm antigen by ELISA 49

Figure 13 Comparison of labelled \& unlabelled Sm antigen by ELISA ..... 49

Figure 14 Fluorescence vs Coomassie for D488-labelled antigens .........51

Figure 15 Comparison of D488 and AF488 labelled antigens ............... 54

Figure 16 Comparison of D488 and AF488 by CIE ......................... 55

Figure 17 Optimisation of electrical current and agarose concentration ...56

Figure 18 Pre-electrophoresis .............................................................57

Figure 19 CIE buffer calcium lactate titration ........................................ 58

Figure 20 Buffer $\mathrm{pH}$ vs Temperature ............................................59

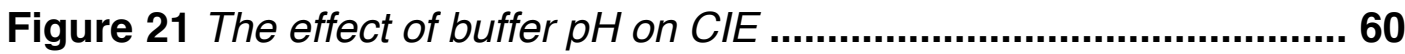

Figure 22 Optimisation of Tris-Tricine concentration ................................61

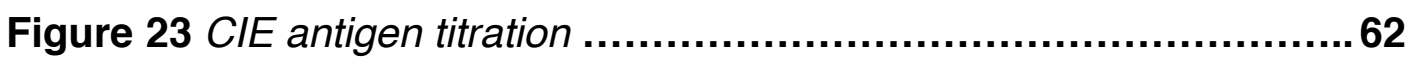

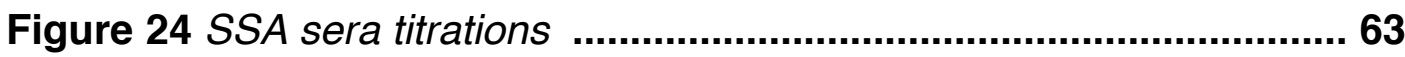

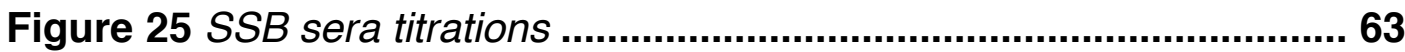

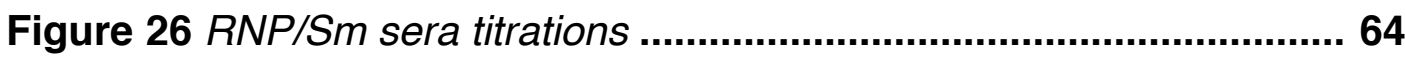

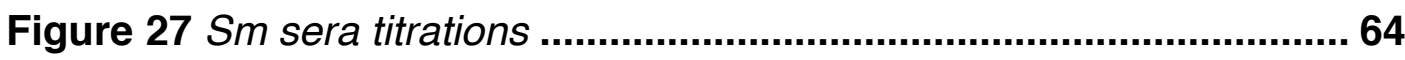

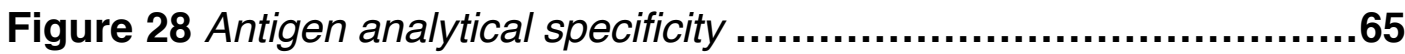

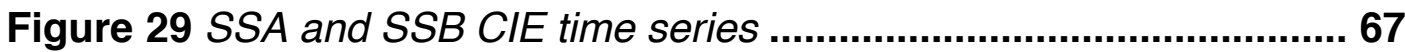




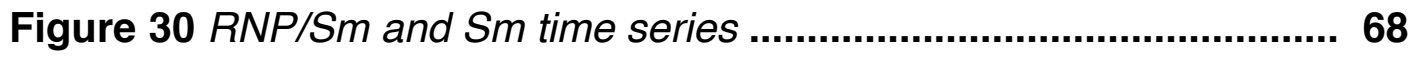

Figure 31 Overlap between CIE and ELISA positive results ................ 70

Figure 32 RNP/Sm DID assays with patient serum samples ................ 74

Figure 33 RNP/Sm DID assays with Arotec internal standards ............. 75

Figure 34 RNP/Sm DID assays with a serum titration series ..................76 


\section{List of Tables}

Table 1. Relationship among statistical terms ........................................................ 16

Table 2. Likelihood Ratio formula .......................................................................... 16

Table 3. Statistical analysis of the diagnostic performance of the ANA IIF assay ..17

Table 4. Relationships between electrical formula............................................ 23

Table 5. Endpoint cutoffs for anti-ENA ELISAs ...............................................69

Table 6. Correlation between SSA CIE and ELISA clinical samples scores ............ 71

Table 7. Correlation between SSB CIE and ELISA clinical samples scores ............... 71

Table 8. Correlation between RNP/Sm CIE and ELISA clinical samples scores ....... 71

Table 9. Correlation between Sm CIE and ELISA clinical samples scores ............. 71

Table 10. Performance characteristics of CIE and ELISA against Case group 1

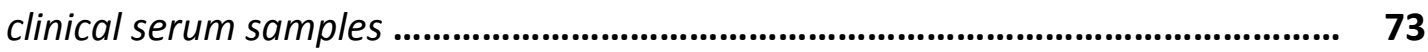

Table 11. Performance characteristics of CIE and ELISA against Case group 2

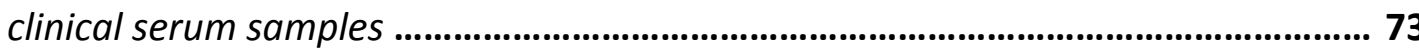





\section{Introduction}

Autoimmunity is when the body's immune system targets the body's own molecular components which then results in an autoimmune disease. There are more than 70 different autoimmune disease disorders that affect approximately $5 \%$ of the population of western countries (Lleo et al., 2010). There can be no discussion of autoimmunity without a grounded understanding of the immune system.

\subsection{The Immune system}

The immune system has evolved a highly complex series of innate and adaptive mechanisms to ward off pathogens and disease, and involves numerous interactions across a wide range of cells, soluble components, and tissues. The innate immune system reacts to possible pathogens in a non-specific manner. The first components of the innate immune system are the physical barriers such as connective tissue and epithelial cells that prevent entry of pathogens into the host body. The innate cellular response is initiated by the recognition of conserved pathogen molecular motifs by pattern recognition receptors (PRRs). The cellular response includes phagocytosis of pathogens and foreign molecules, the release of toxic chemicals (hydrogen peroxide and hypochlorite) to kill pathogens, and the release of inflammatory mediates such as the cytokines tumor necrosis factor and interleukin-1 (IL-1) at the site of infection or trauma (Paul, 2013). The other major component of the innate immune response is the complement system and is a series of circulating proteins that bind to the surface molecules of pathogens. Complement binding in the innate immune response acts either via the alternative pathway, beginning when the C3b complement protein binds to the surface molecule of a pathogen (Paul, 2013) or via the Mannin-binding lectin pathway. This binding attracts the other complement proteins that form a complex initiating one of several immune responses (complement also complexes with surface bound antibodies by the classical pathway).

The adaptive immune system is a highly complex and ordered response to the presence of specific molecule (antigens) within the host. The adaptive immune response begins with the uptake of antigen and presentation of antigenic peptides. Antigens in the body are picked up by the Antigen Presenting Cells (APCs) and 
presented via the major histocompatibility complex (MHC) molecule to the T-cells, activating them and initiating the adaptive immune response. There are two classes of $\mathrm{MHC}$ molecule and the molecule that is used to present antigen depends on the source of the antigen (Paul, 2013). Extracellular antigens such from bacteria, parasites, and toxins that are phagocytosed, degraded, and then presented by $\mathrm{MHC}$ class II: intracellular antigens such as viral proteins, intracellular bacteria, and tumor antigens are digested internally and presented via MHC class I. Antigen presentation via $\mathrm{MHC}$ class II is carried out by dendritic cells (DCs), macrophages, and B-cells; while most cells in the body can present antigen via MHC class I. T-cells interact with the antigen-MHC via specific T-cell receptors (TCRs) on the surface of T-cells (Hsieh et al, 2013). This interaction along with interactions between co-stimulatory molecules/receptors present on APCs and T-cells, as well as soluble factors such as cytokines mediate the activation of T-cells (Paul, 2013). Activated T-cells proliferate and up-regulate the immune response to remove the source of the antigen from the host.

T-cells can be divided into subpopulations based on their role and identifying surface molecules. One of the primary markers of T-cell population subdivision is between the CD8+ and CD4+ surface molecules (Paul, 2013). Naive populations of these cells can be found within the lymph nodes. These cells are activated upon presentation with antigen by APCs. Naive CD4+ T-cells upon presentation with antigen can differentiate into T-helper $\left(\mathrm{T}_{\mathrm{H}}\right)$ cells and depending on the co-stimulatory signal provided by the APC differentiate into $\mathrm{T}_{\mathrm{H}} 1$ or $\mathrm{T}_{\mathrm{H}} 2$ cells (Mills, 2004). $\mathrm{T}_{\mathrm{H}}$ cells primary role in the adaptive immune response is to release cytokines to modify and direct the activity of the other immune cells (Paul, 2013). $T_{H} 2$ cells release the cytokines IL-4, IL-5, and IL-6 which induces B-cell proliferation, antibody (Ab) class switching, and Ab production; $T_{H} 1$ cells produce the cytokine interferon- $Y$ that actives the antimicrobial killing activity of macrophages and induce CD8+ T-cells to proliferate (Mills, 2004). In order for the TCR to interact with MHC class I the T-cell must be CD8+ (Paul, 2013). These CD8+ T-cells are also known as cytoxic T-cells as upon TCR-MHC-class I interaction, and a co-stimulatory signal (either from directly from APCs or indirectly from $T_{H} 1$ ) these cells release a number of cytotoxic chemicals that kill infected cells. There is another group of T-cells known T-regulatory cells $\left(\mathrm{T}_{\text {reg }}\right)$ that are identified by the presence of the FoxP3+ and play a major role in the regulation of 
immunity and autoimmunity as they can down-regulate the immune response at many key steps (Mills, 2004; Roncarolo \& Battaglia, 2007).

$\mathrm{B}$-cells are the antibody $(\mathrm{Ab})$ producing cells of the adaptive immune system. Like the T-cell's TCR the B-cell has a B-cell receptor (BCR) that is able to bind and recognize antigens (Paul, 2013). Unlike the TCR the BCR can recognise native antigen (antigen that has not been processed by an APC) (McHeyzer-Williams \& McHeyzer-Williams, 2005). The development of the B-cell begins with immature B-cells being produced in the bone-marrow (Paul, 2013). They then migrate to the secondary lymphoid tissues and blood. These cells are still naive and although the specificity of their BCR is fixed changes in affinity occur through somatic hypermutation (Goodnow et al., 2005). Upon binding of native antigen to the BC, and followed by co-stimulation from a $T_{H}$ cell within lymphoid tissues, the B-cell undergoes rapid clonal expansion and diversification of its BCR (McHeyzer-Williams \& McHeyzer-Williams, 2005). Diversification of the BCR occurs by a somatic hypermutation of the BCR variable region and creates a pool of BCR receptors each with slightly different affinity for the originally recognised antigen (Melamed et al., 1998). These cells then interact with the $T_{H}$ cell and if the interaction is sufficiently strong, co-stimulatory signals initiate $B$ cell differentiation into either a plasma B cell or a memory B-cell (McHeyzer-Williams \& McHeyzer-Williams, 2005). Both of these cell types having a fixed BCR. When antigen binds to the BCR of the plasma cell, the plasma cell secretes large quantities of antibodies. Memory cells are long lived and are primed to quickly proliferate in response to a secondary exposure to antigen.

The specificity of antibodies produced by a B-cell is the same as its BCR (McHeyzerWilliams \& McHeyzer-Williams, 2005). The role of antibodies is to bind antigens, either individual molecules such as toxins, or antigens on the surface of a cell such as a pathogen or viral proteins attached to an infected cell (Paul, 2013). Antibody bound antigen is recognized by effector cells of the immune system to phagocytose the antigen or to release cytotoxic chemicals. Antibody as previously mentioned also acts as scaffold for complement binding. Another activity of antibody is to localise a foreign body by binding multiple antigen molecules simultaneously, forming a complex with limited mobility within the body. 


\subsubsection{Antigens}

An antigen can be any substance simple or complex, organic or inorganic that elicits, enhances or provokes an adaptive immune response. Antigens are classified into two general types exogenous antigens and endogenous antigens. Exogenous antigens enter the individual from the environment i.e. microorganisms, bacterial, pollens, drugs, and pollutants (Vaman Rao, 2006). Endogenous antigens however are found within the individual and can be sub-divided into three categories.

1. Xenogenic Antigens also known as heterogenic antigens (Vaman Rao, 2006) these are antigens that are common to a number of species. Thus sera will cross-react with cells or fluids of many different species of animal and or microorganisms. The most well-known of these is the Forssman antigen, which is can be found on red blood cells of many species, as well as bacteria (Vaman Rao, 2006).

2. Autologous antigens: These are self antigens and are normally not immunogenic. However these can become immunogenic and cause the host to mount an immune response to its own tissues.

3. Allogenic Antigens; These are antigens from the same species yet due to genetic differences allow differentiation of individuals. An example of this are the $A B O$ blood group antigens.

Generalized properties that influence the level of immunogenicity of an antigen are

1. Foreignness: As a correctly functioning host immune system has generally been screened against self antigens the degree to which the substance is genetically foreign to the host will affect the level of immunogenicity the antigen induces.

2. Molecular size is important as effective immunogens have a size greater than $10 \mathrm{kDa}$ with the best immunogens having a mass of approximately $100 \mathrm{kDa}$ (Vaman Rao, 2006). 
3. Heterogeneity and subsequent chemical composition of an immunogenic molecule is important as synthetic homopolymers are not immunogenic while large co-polymers can be immunogenic especially if aromatic amino acids are added (Bhattacharya \& Sinha, 2006). Also for proteins the primary structure is a primary determinant of immunogenicity with the complex secondary, tertiary, and quaternary structures additionally increasing immunogenicity.

4. The physical form of the antigen is important. Denatured proteins, and insoluble macromolecules are strongly immunogenic as they are more likely to be targeted by the antigen presenting cells (Bhattacharya \& Sinha, 2006).

5. Degradability of a molecule is important for determining the immunogenicity of an antigen (Paul, 2013). An increased ability of APC to degrade a molecule so that has been phagocytosed and have its components present to T-helper cells increases the likely hood it will elicit an immunogenic response.

\subsubsection{Antibodies}

Serum proteins are classified electrophoretically into major groups by increasing isoelectric point (decreasing mobility) as pre-albumins, albumin, alpha-globulins, beta-globulins, gamma-globulins, and basic proteins. In turn these are sub classified by function, molecular size, solubility, and antigenic composition. Antibodies are gamma-globulins and can also be called immunoglobulins (Crowle, 1973). Although the definition of immunoglobulin extends to include molecules having patterns of molecular structure in common with antibodies such as major histocompatibility complex (MHC) and T-Cell receptors which also bind antigen (Elgert, 1996). Immunoglobulins (lg) can be classified into 5 classes $\lg G$, $\lg M, \lg D, \lg A$, and $\lg \mathrm{E}$. Despite their diversity, the family of immunoglobulins have common properties. Circulating antibodies produced are of a polyclonal nature (Vaman Rao, 2006).

In the 1950s and 60s experiments by Porter and Edelman revealed the basic structure of the immunoglobulin molecule for which they jointly received the Nobel prize . Edelman treated the most basic immunoglobulin $\lg G$ with dithiothreitol to disrupt the disulphide bonds in the presence of iodoacetamide to prevent reassociation of the disrupted bonds. This revealed two subunits a heavy $51.6 \mathrm{kDa}$ 
subunit and a light $23.5 \mathrm{kDa}$ subunit. These subunits are known today as the heavy and light chains of the IgG molecule. Edelman also concluded that the complete IgG molecule is $154 \mathrm{kDa}$ and is composed of two heavy subunits and two light subunits (Edelman et al., 1968).

Porter's experiments involved hydrolyzing the peptide bonds of rabbit IgG with the proteolytic enzyme papain in the presence of the reducing agent to cysteine (Elgert, 1996; Kerr, 2006; Porter, 1959). This produced three fragments identified by chromatography. Two of the fragments had similar size $\sim 50 \mathrm{kDa}$ and a very similar amino acid content. These two fragments were named the Fab fragments (fragments of antigen binding) as they retained the ability to bind antigen although they lost the ability to precipitate. The third fragment was heavy and could not bind antigen was named the Fc fragment (fragment crystallisable) for it crystallised during cold storage. Thus, Porter determined that the IgG structure consisted of two similar antigenbinding structures linked to a third structure.

Thus all antibodies consist of two light chains and two heavy chain with the type of heavy chain determining the antibody class. All antibodies have a variable region responsible for antigen binding, and a constant region of which the Fc portion is recognized by the effector cells of the immune system i.e. macrophages. The variable domain of the heavy chain consists of 3 segments labelled V, D, and J. To produce antibodies specific to the infinitely diverse set of possible antigens the body may be exposed to, the genes encoding these segments repeatedly undergo recombination events. This is known as VDJ recombination and produces an equally large set of possible antibodies. The light chain also undergoes similar recombination however there are only two segments $\mathrm{V}$ and $\mathrm{J}$.

\subsubsection{Antigen-Antibody Interactions}

The antibody $(\mathrm{Ab})$ and antigen $(\mathrm{Ag})$ interaction is non-equivalent with the antigenic epitopes interacting with the variable domain of the antibody molecule, particularly the hypervariable region or complement determining region (CDR). The antigenantibody interaction is a non-covalent reaction. It is due to individually weak forces that cumulatively form a strong specific interaction sufficient to prevent dissociation 
but weak enough to allow some reversibility. These weak forces are hydrogen bonds, van der Waals interactions, ionic bonds, and hydrophobic interactions.

However, occasionally weak and often nonspecific unstable antigen-antibody complexes are formed (Vaman Rao, 2006). Non-specific cross reactivity with unrelated antibodies is possible only when two different antigens share similar epitopes or antibodies specific for one epitope also bind to an unrelated epitope possessing similar chemical properties (Vaman Rao, 2006).

Affinity at one binding site does not always reflect the true strength of the antibodyantigen interaction. The strength of multiple interactions between a multivalent antibody ( $\operatorname{lgM}$ ) and antigen is called avidity, as opposed to affinity, which describes the strength of single interaction at one binding site. The avidity of an antibody is its capacity to form a stable complex. It is observed that high avidity compensates for low affinity as is the case of IgM has low affinity but has high avidity because of its 10 weak binding sites (Vaman Rao, 2006).

\subsubsection{Tolerance}

The immune system is able to differentiate between self and non-self components. This is known as self tolerance and is mediated by a complex process known as clonal selection that augments $\mathrm{T}$ and $\mathrm{B}$ lymphocytes populations to discriminate between self and non self antigens. Clonal selection of T-lymphocytes occurs in the thymus and has two primary steps (Klein et al., 2009). In the first selection step the lymphocyte TCR is exposed to a antigen-MHC complex expressed on cortical thymic epithelial cells and selection is based on the TCR's affinity for antigen-MHC. TCRs that have a moderate to high affinity for antigen-MHC are positively selected and move on to the second stage of selection: those with no or very low affinity for antigen-MHC are neglected and die (apoptosis). In the second step of selection the TCRs are exposed to MHC bound self antigens that are expressed by medulla cortical thymic epithelial cells and antigens presented by DCs. TCRs that have a moderate to high affinity to the antigen-MHC complex are actively deleted by apoptosis (negative selection). The majority of the remaining T-cell population has a low to moderate affinity for $\mathrm{MHC}$ and no affinity for self antigens. There does remain a small population of T-cells that have a low affinity for self antigen and post- 
thymically they develop into $T_{\text {Reg }}$ cells that play a major role in regulating immunity and suppressing autoimmunity (Mills, 2004).

The B-cell lymphocytes also go through a process of clonal selection throughout their development (Goodnow et al., 2010). The BCR of a naive B-cell in the bone marrow that binds self antigen will halt its maturation, internalise the BCR, and initiate VDJ recombination. Unless the B-cell can produce a new BCR that is not self reactive the cell will die in 1-2 days (Goodnow et al., 2005). After leaving the marrow, B-cells acquire sensitivity to antigen-mediated apoptosis, such that if antigen binds to the $\mathrm{BCR}$ without a co-stimulatory signal the B-cell will possibly undergo paralysis followed by apoptosis (Melamed et al., 1998). However if the B-cell receives a costimulatory signal from a $T_{H}$ cell after binding the antigen the co-stimulatory signal will cause the B-cell to proliferate instead of arrest, since the co-stimulatory signaling relies on the $T_{H}$ cell to have undergone thymic selection and not be self-reactive.

\subsection{Autoimmunity and autoimmune disease}

\subsubsection{Loss of tolerance and autoimmunity}

Some individuals lose tolerance as the fine-tuning of the immune system to self and non-self antigens fails and leads to an autoimmune disease. Although a number of genetic sequences have shown to be associated with autoimmunity they have at this point provided little clinical significance (Selmi, 2011). Rather, the loss of tolerance is attributed to a complex interaction between genetics, and what are mostly unspecified environmental factors such as drug interaction, and infection (GomezPuertaet al, 2008; Lleo et al., 2010). Although there is little evidence showing specific mechanisms that induce a specific autoimmune disease, there are a number of known mechanisms of tolerance failure.

There are several mechanisms of activation of self-specific $B$-cells via $T_{H}$ cells. It has been shown that B-cells bind to membrane bound $\lg G$ and produce $\lg G$ autoantibodies (rheumatoid factors) that can be activated by $T_{H}$ cells after the B-cell has phagocytosed an Ag-Ab complex (Roosnek \& Lanzavecchia, 1991). The key to this is that when the B-cell with a BCR specific for the $\lg G$ antigenic peptide, 
recognises and phagocytoses the associated $\mathrm{Ag}-\mathrm{Ab}$ complex, the peptide that is presented by the $\mathrm{B}-\mathrm{Cell}$ to the $\mathrm{T}_{\mathrm{H}}$ cell can be any peptide present in the $\mathrm{Ag}-\mathrm{Ab}$ complex. Similarly $\mathrm{T}_{H}$ cells that have been activated and are responding to foreign antigen can be misdirected to self reactive BCRs that cross-react with a component of the foreign antigen (Goodnow et al., 2005). This concept that a foreign antigen, sharing some similar epitopes to a self antigen, can induce autoimmunity is known as molecular mimicry. A well known example of autoimmunity induced following bacterial infection and attributed to molecular mimicry is that of rheumatic fever. However, no specific antigen has been identified as the cause.

It is known that bacterial macromolecules e.g. lipopolysaccharides can act as ligands for the co-stimulatory receptor substituting the T-cell signal needed for B-cell proliferation (Goodnow et al., 2005). The substitution of co-stimulatory signals by some bacterial antigens can also cause T-cell activation by activating immature DCs to release T-cell activating signals or by directly by passing the co-stimulatory signals of APCs (Mills, 2011). The activation of immune cells through a co-stimulatory signal is the theoretical mechanism attributed to adjuvant function.

Problems in BCR editing have been associated with autoimmune disease. In the case of the autoimmune disease systemic lupus erythematosus (SLE), most of the anti-double-stranded (anti-DS) DNA antibodies are somatically mutated indicating a problem with regulation of self-reactive receptors (Goodnow et al., 2005). Additionally, anti-nuclear and anti-DS DNA autoantibodies like those found in SLE are more frequently borne by a subset of B-cells with little or no surface BCR in human bone marrow (Goodnow et al., 2005). This indicates that there has been some failure to edit or delete these self-reactive BCR-producing B-cells.

The role of $T_{\text {Reg }}$ cells is to suppress the immune response produced other selfantigen reactive immune cells. As such, defects in $\mathrm{T}_{\text {Reg }}$ cells are associated with autoimmune disease and experimental suppression of $T_{\text {Reg }}$ activity has been shown to exacerbate autoimmune effects within the host (Roncarolo \& Battaglia, 2007). 


\subsubsection{Autoimmune diseases}

Autoimmune diseases can be divided into two broad categories organ specific and systemic autoimmune diseases (Vaman Rao, 2006). In an organ specific autoimmune disease the immune response is directed against a specific antigen corresponding to a single organ. There are several mechanisms of organ specific autoimmune disease such as humoral or cell mediated attack of the organ causing severe cellular damage as is the case in type 1 diabetes where by the immune system targets and destroys the insulin producing beta-cells of the pancreas, thus inducing diabetes (Paul, 2013). Rather than destroying organ function, auto-immunity may induce hyperactivity of an organ. In Graves' disease autoimmunity induces hyperthyroidism as autoantibodies bind to the thyroid-stimulating hormone receptors (TSH-Rs), and induce thyroid hormone production and thyroid growth (Paul, 2013). Autoantibodies may conversely block a function preformed by the cell or organ as in the case of Addison's disease (Vaman Rao, 2006) whereby autoimmune destruction of the adrenal cells results in insufficient hormone production.

For systemic autoimmune diseases, conversely the autoantibodies are directed against broad range of antigen and target organs. This is caused by defective immune regulation of $T$ and $B$-lymphocytes, which leads to widespread tissue damage from cell mediated and humoral antibodies as well immune complex mediated response (Vaman Rao, 2006). Examples of such diseases are connective tissue diseases such as SLE, systemic sclerosis (SSc), Sjogren's syndrome (SS), polymyositis/dermatomyositis (Abbreviated PM/DM and also known as idiopathic inflammatory myopathies). 


\subsection{Autoantibodies}

The detection of specific antibodies in the serum of patients is a pillar of diagnosis and management for some autoimmune diseases. For connective tissue diseases (CTDs) such as SLE the detection of autoantibodies to specific nuclear antigens is used as a primary clinical diagnostic feature. Antibodies that react with nuclear antigens are known as antinuclear antibodies (ANAs). The presence of specific ANAs can be used as a predictive marker for the disease and patient outcome (GomezPuerta et al., 2008; Hernandez-Molina et al., 2011), particularly as patients can present autoantibodies many years before they show clinical symptoms of disease. Anti-RNP antibodies and a lack of kidney involvement in SLE is an indicator of a favorable patient outcome (Shoenfeld \& Tincani, 2005). However, changes in autoantibody titer can often do not correlate with a patient's clinical situation: low ANA titer is associated with SLE, high titers can found in healthy patients (Shmerling, 2005), and autoantibodies can persist when a patient is in remission (Shoenfeld \& Tincani, 2005). This lack of correlation between disease and antibody titer may be because some autoantibodies such as many of 116 associated with SLE are not actually a causative agent of the autoimmune disease but are rather an epiphenomenon (Shoenfeld \& Tincani, 2005). This can make the clinical utility of autoantibody detection questionable. In a comprehensive review by Sheldon (2004) it was recommended that autoantibodies be considered markers of disease and only tool that is part of a larger diagnostic panel.

It is common for patients displaying symptoms of autoimmunity to test positive for more than one autoantibody (Defendenti et al., 2011). Anti-SSA (also known as antiRo) and/or anti-SSB (also known as anti-La) antibodies are the primary serological markers in the diagnosis of SS and SLE with patients presenting precipitating antibodies in $60 \%$ and $40 \%$ respectively of SS patients, and $35 \%$ and $15 \%$ respectively of SLE patients (Manoussakis et al., 1995; Reichlin \& Harley, 1986). Also, in the case of SS, the antibody profile is fixed at an early stage of the disease (Hernandez-Molina et al., 2011). Anti-SSA antibodies are also associated with 3-11\% of patients with SSc and 5-15\% of patients with PM/DM (Franceschini \& Cavazzana, 2005). The SSA complex is a ribonuclear protein complex containing hY-RNAs, and $52 \mathrm{kDA}$ and $60 \mathrm{kDA}$ protein subunits. The SSB molecule is composed of a highly 
conserved $48 \mathrm{kDa}$ nuclear protein that is capable of associating with various small RNAs, including precursor transcripts of RNA pol III (Manoussakis et al., 1995). The Ro/SSA complex has been identified as an E3 ligase that negatively regulates cytokine production induced by the IFN-gamma pathway and the SSB protein works as a transcription termination factor of RNA polymerase III (RNA pol III) (HernandezMolina et al., 2011).

Other ANA include anti-Sm which is seen in $25-30 \%$ of SLE cases (Colglazier \& Sutej, 2005). The anti-Sm antibody binds to a series of 7 small proteins that complex with 4 small RNAs to form a small nuclear ribonucleoprotein (snRNP) complex (Migliorini et al., 2005). The anti-RNP antibody binds the U1-RNP complex is another snRNP composed of 3 proteins and the U1-RNA (Sheldon, 2004). The Sm and RNP snRNP complexes are found in the nuclei of all eukaryote cells and function together as a spliceosome (Migliorini et al., 2005). The anti-Sm antibodies are associated with approximately $30 \%$ of North American and $5 \%$ of European SLE patients and antiRNP antibodies are detectable in 25-47\% of all SLE patients (Migliorini et al., 2005). The anti-RNP antibodies are associated with mixed connective tissue diseases consisting of a combination of SLE, SSc, and polymyositis: the nature of mixed connective tissue disease means the prevalence of anti-RNP antibodies cannot be calculated (Colglazier \& Sutej, 2005). Anti-RNP and anti-Sm antibodies can also be found in $2 \%$ and $1 \%$ respectively of SS patients (Nardi et al., 2006). Like SSA and SSB it is common for patients to be have both anti-RNP and anti-Sm antibodies, with anti-Sm nearly always associated with anti-RNP antibodies (Migliorini et al., 2005). Anti-Scl-70 antibodies target topoisomerase I and occur in $20 \%$ of patients with diffuse SSc (Colglazier \& Sutej, 2005). Anti-Jo-1 antibodies target the histidyl-tRNA synthetase and their presence is found in interstitial lung disease and PM/DM (Bernstein et al., 1984). 


\subsection{Antinuclear antigen antibody testing}

\subsubsection{Whole cell screening of ANAs}

In 1948 the L.E. cell was identified and described in the bone-marrow preparations of patients with SLE (Hargraves et al., 1948). The lupus erythematous cell (LE cell) is a polymorphonuclear leucocyte that contains cell nuclei that have been phagocytosed and digested (Hepburn, 2001). In 1949, experiments by Haserick and Bortz added plasma from patients with SLE to bone marrow preparations from normal subjects and these results were compared to bone-marrow preparations using plasma from the same healthy subjects (Haserick \& Bortz, 1949). The plasma from patients with SLE induced the LE cell phenomenon while plasma in the control group failed to produce the phenomenon. The LE cell phenomenon was used as a primary diagnosis test for SLE (Hepburn, 2001).

The whole cell detection of ANAs is now done by an indirect immunofluorescence (IIF) assay (Hepburn, 2001: Keren, 2002). In this assay Hep-2 cells are fixed on a slide and then incubated with patient serum. After washing a conjugated anti-human antibody labelled with a fluorophore is then added and binds to the patient autoantibodies bound to the cell nucleus. Slides are examined using fluorescent microscopy to examine the nuclear fluorescent staining pattern. The type of staining pattern correlates with patient diagnosis and can be used to diagnose autoimmune disease (Keren, 2002). A homogenous staining pattern indicates anti-DNA antibodies and is associated with SLE A diffuse staining pattern indicates either anti Scl-70 antibodies that are associated with SSc, or anti-histone antibodies that are associated with SLE and drug induce lupus (Sheldon, 2004). A speckled staining pattern indicates the presence of antibodies to extractable nuclear antigens (ENAs) such as RNP, Sm, SSA, and SSB (Sheldon, 2004). A cytoplasmic staining pattern is associated with anti-Jo-1 antibodies (Sheldon, 2004).

The performance of ANA screening by IIF is difficult to assess as the method, substrate, and population subset differs between laboratories (Colglazier \& Sutej, 2005; Solomon et al., 2002). ANA IIF screening also tends to produce a high number of false positives and this limits its utility as a screening test (Colglazier \& Sutej, 
2005; Solomon et al., 2002). ANA IIF screening can also miss some critical antigen specificities notably anti-SSA and anti-Jo-1, as the antigens are poorly expressed or are not conserved in the cell line (Dahle et al., 2004; Sheldon, 2004). Regardless of these flaws, ANA IIF screening is still a widely used technique for the detection of ANAs.

\subsubsection{Detection of autoantibodies to extractable nuclear antigens}

There are several diagnostic techniques available for the detection of autoantibodies using ENAs rather than using whole cells. These include gel immunoprecipitation methods, double immunodiffusion (DID) and counter immunoelectrophoresis (CIE), the enzyme-linked immunosorbent assay (ELISA), the line immunoassay (LIA), immunoblotting (IB), and RNA precipitation. None of the methods can be considered to be a gold standard diagnostic method as each technique has practical and analytical advantages and disadvantages in respect to the other techniques. Also the standardisation of the techniques is difficult due to:variation in the antigen source and preparation; variations in antibody affinity in patient samples and standard samples; differences in methodology between laboratories (Sheldon, 2004).

The prevalence of anti-ENA antibodies is low in the general population (Shmerling, 2005) and current tests have a low positive predictive value and thus it is recommended that for patients suspected of having an autoimmune CTD that there be a two stage testing process (Phan et al., 2002): An initial highly sensitive screening test to identify all possible autoimmune patients with anti-ENA antibodies, followed by a highly specific assay (low false positives) that will confirm the presence of anti-ENA antibodies 


\subsection{Comparison of diagnostic screening methods}

\subsubsection{Statistical considerations}

In order to determine whether an assay is of clinical utility as a diagnostic screening test a number of statistical analyses can be done. The diagnostic sensitivity and specificity of an assay (Table 1) are two crucial statistical parameters used to assess the practical effectiveness/accuracy of an assay, as they are independent of disease prevalence. Diagnostic sensitivity measures the proportion of positives that are actual true positive results. Diagnostic specificity measures the proportion of negative results that are actually true negatives.

Another set of useful statistics for assessing the diagnostic utility of an assay are positive predictive value (PPV) that measures the proportion of samples that are true positives, and the negative predictive value (NPV) that measures the proportion of true negatives. Thus these two statistics are measure of diagnostic perfomance. However PPV and NPV are only applicable to the study population from which they were derived and the PPV and NPV quoted for one study's population sample are consequently not directly transferable to another study's sample population (Phan et al., 2002). PPV and NPV should only be used to assess an assay if the number of the true positives (truly healthy patients) and the number of true negatives (patients that truly have the disease) used during diagnostic testing matches the prevalence of the disease in true population.

The likelihood ratio (LR) should be used instead of than NPV and PPV, as it depends on the prevalence of the disease. There are two LRs that can be calculated (Table 2). The positive LR (PLR) is the probability of a person who has the disease testing positive and the negative LR (NLR) is the probability of a person with the disease testing negative. For example, a PLR of 5 indicates that a person with the disease is 5 times more likely to produce a positive result than a person without the disease. LR values higher than 10 and less than 0.1 indicate a positive or negative confirmatory diagnoses respectively and LR values close to 1 provide no clinical value as the test does not change the odds of identifying the disease (Deeks \& Altman, 2004). 
In reporting the agreement between different methods of anti-ENA detection the kappa-statistic should be used (Phan et al., 2002). The kappa-statistic gives a measure of how strongly the inter-rater agreement is between two observations, such as the agreement of results between two different assays. A strong agreement indicates that any difference between two observations (assay results) is not caused by the observers (assay techniques). A weak agreement indicates that the difference in observations is due to the observers. The Cohens kappa value can be interpreted as follows; 0 as indicating no agreement, $0-0.2$ as slight, $0.21-0.4$ as fair, $0.41-0.6$ as moderate, $0.61-0.8$ as substantial, and $0.81-1$ as strong agreement (Kraemer, 1980; Landis \& Koch, 1977).

\begin{tabular}{|c|c|c|c|c|}
\hline & & \multirow{2}{*}{\multicolumn{2}{|c|}{$\begin{array}{l}\text { Patient condition as determined by } \\
\text { clinical benchmark }\end{array}$}} & \\
\hline & & & & \\
\hline & & Condition Positive & Condition Negative & \\
\hline \multirow{2}{*}{$\begin{array}{l}\text { Patient test } \\
\text { results from the } \\
\text { diagnostic test } \\
\text { being evaluated }\end{array}$} & $\begin{array}{c}\text { Test } \\
\text { Positive }\end{array}$ & True Positive (TP) & False Positive (FP) & $\begin{array}{c}\text { Positive predictive value } \\
\text { = TP } /(\mathrm{TP}+\mathrm{FP})\end{array}$ \\
\hline & $\begin{array}{c}\text { Test } \\
\text { Negative }\end{array}$ & $\begin{array}{l}\text { False Negative } \\
\qquad \text { (FP) }\end{array}$ & True Negative (TN) & $\begin{array}{c}\text { Negative predictive value } \\
=\text { TN } /(\text { TN }+ \text { FN) }\end{array}$ \\
\hline & & $\begin{aligned} & \text { Sensitivity } \\
= & \mathrm{TP} /(\mathrm{TP}+\mathrm{FN})\end{aligned}$ & $\begin{array}{c}\text { Specificity } \\
=\mathrm{TN} /(\mathrm{TN}+\mathrm{FP})\end{array}$ & \\
\hline
\end{tabular}

Table 1. Relationship among statistical terms

Positive Likelihood Ratio (PLR) = Sensitivity / (1 - Specificity)

Negative Likelihood Ratio (NLR) = (1 - Sensitivity) / Specificity

Table 2. Likelihood Ratio formula

\subsubsection{ANA IIF}

Soloman et. al. (2002) collated and evaluated the published data on ANA IIF diagnostic testing. The calculated sensitivity, specificity, PLR, and NLR from this data (Colglazier \& Sutej, 2005) for the diagnosis of select autoimmune diseases by ANA 
IIF assay is shown in Table 3. Based on this data the sensitivity and PLR indicate that for SLE and SSc the ANA IIF assay would be suitable as an initial screening test. However all 4 CTDs have low specificity, a low NLR and thus have a high probability of producing a false positives result. These results confirm the need for a secondary alternative high specificity assay for CTD diagnosis. ANA IIF is also a labor intensive and time consuming assay that produces a subjective result (Keren, 2002).

\begin{tabular}{lcccc}
\hline Disease & Sensitivity & Specificity & $\begin{array}{c}\text { Positive } \\
\text { likelihood ratio }\end{array}$ & $\begin{array}{c}\text { Negative } \\
\text { likelihood ratio }\end{array}$ \\
\hline Systemic lupus erythematosus & $93 \%$ & $57 \%$ & 2.2 & 0.11 \\
Scleroderma & $85 \%$ & $54 \%$ & 1.86 & 0.27 \\
Polymyositis/Dermatomyositis & $61 \%$ & $63 \%$ & 1.67 & 0.61 \\
Sjogren syndrome & $48 \%$ & $52 \%$ & 0.99 & 1.01
\end{tabular}

Table 3. Statistical analysis of the diagnostic performance of the ANA IIF assay

Colglazier \& Sutej, 2005

\subsubsection{ELISA}

ELISA kits have become the most widely used method for anti-ENA antibody testing (Phan et al., 2002). A comparative study of 5 commercially available ELISA kits for anti-ENA testing indicated that ELISA is comparable and is some cases superior to ANA IIF (Tonuttia et al., 2004). ELISA assays have a number of inherent advantages that include rapid turnaround time, objective and quantitative results, and can be automated. ELISA is considered highly a sensitive assay for ENA detection with a lack of specificity comparably to other methods being its major flaw (Phan et al., 2002). Also pure antigen is required or issues regarding lack of specificity are exacerbated (Phan et al., 2002).

\subsubsection{Immunoblotting}

The major advantage of IB is its ability to differentiate antibody interactions between different subunits of the same antigenic complex most notably between the 60-kDa and $52 \mathrm{kDa}$ SSA subunits (Phan et al., 2002). A Loss of sensitivity is a problem for IB as proteins are denatured and therefore lose their conformational epitopes.

Conformational epitopes are antibody binding sites that require tertiary and/or 
secondary protein conformations to remain intact. A conformational epitope can form when a protein folds and peptides separated by sufficient space to prevent a specific antibody interaction are brought into close proximity to one-another allowing the antibody to bind. IB is also relatively expensive, and time consuming technique compared to CIE (Phan et al., 2002).

\subsubsection{RNA precipitation}

RNA precipitation is considered to have the highest sensitivity and specificity for the detection of anti-SSA and anti-SSB antibodies (Franceschini \& Cavazzana, 2005). A comparative analysis of RNA precipitation, ELISA, IB, and CIE also identified RNA precipitation as having the highest specificity for anti-SSA and anti-SSB antibodies (Meilofet al, 1990). However the specificity and sensitivity of the CIE assay are comparable to those of the RNA precipitation assay. The authors also concluded that CIE was a better assay for routine diagnostics. A similar study performed by Manoussakis (1993) came to similar conclusions (Manoussakis et al., 1993) and both papers also reporting low specificities for ELISA.

\subsubsection{Immunoprecipitation; DID and CIE}

Immunoprecipitation methods have traditionally been a widely used method for the detection of ENA antibodies as they are highly accurate (Manoussakis et al., 1995). The quality of antigenic extract required for DID and CIE is quite robust and it is possible to use crude antigen homogenates for detection (Kveder \& Bozic, 2002). CIE and DID have been shown to be a highly specific assay methods (Phan et al., 2002). A comparative study by Phan et al (2001) of the CIE, ELISA, LIA concluded that the overall cost effectiveness of CIE is far superior to that of ELISA, and LIA (Phan et al., 2001). Also anti-ENA detected by CIE correlated better with their respective clinical diagnosis than anti-ENA detected by LIA and ELISA (Phan et al., 2001).

Manoussakis et al. (1995) compared the diagnostic sensitivity and specificity of CIE and Immunoblot to detect SSA and SSB antibodies in patients with SS and SLE. In this study detection of SSA and SSB for both CIE and immunoblot assays was shown to be highly specific (100\%) for SS and SLE. Diagnostic sensitivity for SSB was the 
same in both SS and SLE. However, the diagnostic sensitivity of the CIE assay was superior to immunoblot for the detection of SSA in both SS and SLE patients.

The downside to both DID and CIE are that they are labour intensive and interpretation of results is dependent on the skill of the technician performing the assay. SSB due to a lack of conformational epitopes, and a low frequency of linear epitopes that tend to have a low antigenicity result in a reduced ability to precipitate and thus be detected by CIE or DID (Phan et al., 2002). The nature of the CIE assay itself can lead to a reduction in sensitivity for specific antigens. For example the Scl70 antigen is less negatively charged than other antigens and thus tends to move in the same direction as the patient antibodies towards the cathode. A technical advantage CIE has over DID is the time taken to reach assay completion (although this is dependent on the exact methodology of the two techniques). Also the sensitivity of CIE is superior to DID for the detection of SSA (Franceschini \& Cavazzana, 2005).

\subsection{Counter Immunoelectrophoresis}

Counter immunoelectrophoresis also known, as countercurrent immunoelectrophoresis, is a rapid method used to examine reactivity between antigen and antibody. CIE takes advantage of the charge of the antigen and endosmotic flow to move antigen and antibody in opposite directions simultaneously so that analogous to a double diffusion test, they will meet, react and precipitate somewhere between their respective origins (Fig. 1) (Crowle, 1973). For this technique to work the antigen must be negatively charged, as to be electrophoretically drawn towards the anode and the antibody must be neutrally charged to be drawn by endosmotic flow towards the cathode.

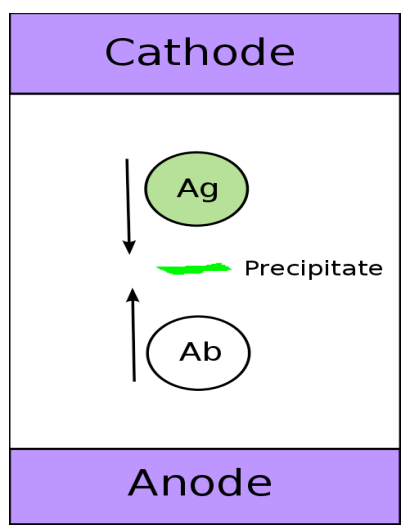

Figure 1 Diagrammatic representation of antigen and antibody migration during CIE The antigen $(\mathrm{Ag})$ electrophoretically migrates towards the anode. The antibody (Ab) is drawn towards the cathode my endosmosis. At the point the $\mathrm{Ag}$ front and the $\mathrm{Ab}$ front meet a precipitate forms. 
There are many advantages to CIE over immunodiffusion methods; it is faster, more economical, more sensitive, can used with antigen molecules too large to simply diffuse quickly through agar gels and it can detect inefficient antibody which routine immunodiffusion tests form too diffuse and ephemeral a precipitate to be seen (Crowle, 1973).

$\mathrm{CIE}$ although very rapid has its flaws. First there is little time for the visible secondstage antigen-antibody complex to accumulate and result in precipitation. Also, both reactants migrate as finite zones (that match the shape of their origins) that rapidly collide with each other. This results in a limited range of antigen-antibody ratios that can occur during the brief interaction. Thus the optimal ratio of antigen to antibody required for precipitation (the equivalence zone) may not be achieved and so CIE is highly susceptible to adverse conditions of antibody and antigen excess (Crowle, 1973). Also the wells need to be in line with each other to ensure the migrating zones don't miss each other. Another disadvantage of CIE is due to the forces driving antibody and antigen in opposite directions precipitation may occur briefly and then reverse before it is been noticed (Crowle, 1973). Some of these disadvantages can be minimized mechanically by using a lower voltage or higher ionic strength to lower the rate of migration; elongating wells increases the size of the migrating zone and thus creates a gradient of antibody-antigen interaction that will improve the chances of reaching equivalence.

\subsection{Chemical and physical processes}

\subsubsection{Precipitation reactions}

Precipitins are antibodies which become insoluble after forming an antigen-antibody complex (Crowle, 1973). The reaction between soluble antigen and antibody leading to a visible precipitate is called the precipitin reaction or the precipitation reaction. The origin of precipitation reactions for the specific purpose of analysis of animal serum were developed by Oudin at the Pasteur Institute of Paris (Arquembourg, 1975). Soluble antibody (animal serum) and antigen (proteins) were loaded into separate wells punched into a gel. The antigen and antibody were allowed to diffuse 
through the gel, until the antigen and antibody diffusion fronts met. A visible precipitate would form due to the antigen and antibody interacting to form a complex. Ouchterlony added a second dimension to gel reactions; a third well that allowed for analysis of antigens from different sources (Arquembourg, 1975). Now known as Ouchterlony double diffusion, this technique allowed the identification of crossreactivity of the same antibody with different antigens (Identity), the identification of multiple antibodies in the same serum sample interaction with different antigens (nonidentity), and the identification of a single antibody recognizes common parts in structure between antigens from different sources, which are, for the rest of their constituents, different (partial identity) (Arquembourg, 1975).

In the first stage of a precipitin reaction begins with the initial formation of a variety of invisible complexes. These complexes form as antibody and antigen collide and antibody binds to one of several ligands usually present on each antigen molecule. This is followed by formation of a lattice of soluble complexes that gradually expands into a visible precipitate due to antibodies interacting with the ligands of two separate antigens (Arquembourg, 1975; Crowle, 1973). In order to achieve antibody-antigen interactions at multiple reactive sites the antibody needs be bivalent, i.e. more than one Fab site should be available for reacting with antigen. Similarly antigen should be bivalent or polyvalent, i.e. it must have multiple epitopes available that can simultaneously react with different antibodies (Vaman Rao, 2006).

Antigen-antibody reactions are not stoichiometric and in order to form a visible antigen-antibody precipitin, the concentrations of both antigen and antibody play an important role. An excess of either antigen or antibody will cause combining sites to become saturated and lead to insufficient lattice formation needed for precipitation (Arquembourg, 1975). When the antigen antibody ratio is optimal maximum precipitate will form which is called equivalence zone (Vaman Rao, 2006). When antigen-antibody precipitin reactions occur in a gel, a visible arc will form. The visibility of the precipitin arc will be greater at the center of the arc where optimal/equivalent antibody-antigen conditions exist, and visibility will fade towards the tips of the arc as the condition of antibody excess increases towards the edges of the precipitin arc (Arquembourg, 1975). 
There are a number of other factors that affect the complex precipitation reaction that takes place between antigen and antibodies. These factors include non-antibody serum constituents such as lipids and serum complement proteins that improve precipitation; physiochemical conditions that fall outside optimal ranges of $\mathrm{pH}$, salt concentration, and temperature antagonize precipitin formation; other non precipitating antibodies that have a weak avidity/affinity and possibly are non-specific for the antigen have been shown to help stabilize lattice formation (Crowle, 1973). Also certain ions such as $\mathrm{Cd}, \mathrm{Ni}$ and barbital which precipitate lipids enhance $\mathrm{Ag}-\mathrm{Ab}$ precipitation, and buffers that chelate such ions, like EDTA, Tris or citrate,depress it (Crowle, 1973).

In the analysis of a complex mixture of antigens, gel diffusion reactions produced a series of precipitin bands concentrated in a small area in which recognition was impossible. Pierre Grabar then at the Pasteur Institute of Paris opened a third dimension. By submitting the antigen mixture to electrophoresis before carrying out the double immune diffusion reaction he separated the complex series of bands into a set of independent double diffusion reactions at locations imposed by ionization characteristics of antigens. (Arquembourg, 1975).

\subsubsection{Electrophoresis}

Electrophoresis can be defined as the movement of charged particles by direct current through an electrolyte solution. The basic apparatus required for gel electrophoresis consists of a power supply for direct current, positive and negative preferably non-polarizing electrodes, two vessel of buffer for immersing each electrode, that are separated by the supporting material (gel) in which electrophoresis will occur.

When using gel electrophoresis the electrochemistry of the technique must be taken into account. In gel electrophoresis procedures, voltage (V) is the difference in electric potential between the two electrodes is held constant and the rate of migration of a molecule away from its point of origin is proportional to the voltage applied (Crowle, 1973). With voltage held constant the current (I) is determined by the conductance $(\mathrm{G})$ of the buffer and medium divided by applied voltage. Resistance $(R)$ is the reciprocal of conductance so any change in conductance will result in a 
change in resistance. Wattage (W) in this instance can be considered a measurement of thermal output (heat) and will rise with any increase in current or resistance; however changes in current will have a greater exponential impact on temperature compared to resistance.

$$
\begin{aligned}
& V=I R \\
& G=I / V \quad R=V / I \\
& I=G / V \\
& W=I^{2} R
\end{aligned}
$$

\section{Table 4. Relationships between electrical formula}

There are many factors that affect and need to be controlled in gel electrophoresis. Heat is a major consideration, as excessive heating will cause a number of problems. Such problems include disruption of the precipitin reaction, and evaporation of water leading to drying, shrinkage, and distortion of the electrophoretic gel medium. As previously mentioned, current is the main determining factor for temperature (Wattage). Thus, we can ascertain from the formula I = G N (Table 4) that if voltage is constant, in order to keep current low, a buffer with low ionic strength (ionic strength is relative to conductance $(\mathbf{G})$ ) is needed. Importantly if evaporation occurs, solute concentration increases leading to an increase in current and thus an increase in temperature.

The $\mathrm{pH}$ of the medium and buffer is important as it determines the charge of the proteins being electrophoresed. The overall net charge of a protein determines its direction and rate of movement through the medium. A protein composed of aminoacids with both acidic (carboxyl) and basic (amino) groups exposed can have a positive, negative, or neutral charge and needs to have a net electronegative charge in order to move towards the anode (Crowle, 1973). For this to occur the $\mathrm{pH}$ of the buffer needs to be higher than the isoelectric point of the protein. The isoelectric point is the $\mathrm{pH}$ at which a molecule carries no net electric charge i.e. it is neutrally charged. During electrophoresis $\mathrm{pH}$ change occurs as buffer salts are moved by electrolysis towards the cathode and anode driven by the current passing through the medium. Normally this is buffered well enough to prevent any significant $\mathrm{pH}$ change that will affect either the precipitation reaction or net charge of the proteins but if the current is excessive or buffering capacity is poor a significant change can occur (Crowle, 1973). 
Also the flow of solutes towards the termini leads to an increase in their local concentration. Thus leading to local hotspots and increased evaporation at the termini (Crowle, 1973).

The composition and physical characteristics of the gel medium all affect electrophoresis. The movement of molecules through the supporting medium is inversely proportional to the density of the medium. There are a number of supporting mediums commonly used for gel electrophoresis agar, agarose, polyacrylamide, starch etc. When choosing a support medium one must take into account its physical characteristic such as pore size and chemical composition. Molecules can interact and weakly bind to the supporting mediums charged groups affecting both migration and separation of molecules in gel electrophoresis. For the purpose of immunoelectrophoresis agarose is considered a better medium than Agar (Crowle, 1973). Also the charged groups of the supporting medium can cause the phenomena endosmosis that impedes molecule migration.

\subsubsection{Endosmotic Flow}

During electrophoresis as ions move either towards the cathode or anode they drag along molecules of water with them. In a basic or neutral buffer the negatively charged groups of the supporting medium such as carboxylic groups in starch and agarose, sulfonic groups in agarose, silicon oxide on glass surfaces are fixed in place and thus cannot move towards the cathode (Westermeier, 2001). However their positively charged counter-ion can move towards the cathode. This results in a net migration or flow of water towards the cathodic side of gel. This flow of water carries carries neutrally charged solubilised substances along with it.

The endosmotic flow is also known as electroendosmosis and the flow of water towards the cathode physically impedes the migration of negatively charged particles migrating to the anode. In addition buffer leaves the strip or gel support faster than it can re-enter at the anode side. This results in a drying on the cathodic side and in gel electrophoresis a thinning of the gel (Arquembourg, 1975). Increasing buffer conductivity increases the strength of the endosmotic flow and thus often a buffer of lower ionic strength is used to prevent endosmostic flow and increase migration speed at the expense of gel resolution (Arquembourg, 1975; Verbruggen, 1975). 


\subsubsection{Immunoelectrophoresis}

Immunoelectrophoresis is the name given to a group of techniques by which gel electrophoresis is used to force together antibody and antigen in order accelerate antibody-antigen precipitation and allow greater separation of serum components than other immunodiffusion tests (Crowle, 1973). The first immunoelectrophoretic technique was developed by Pierre Grabar at the Pasteur Institute of Paris. Unable to differentiate by double immunodiffusion a series of concentrated precipitin bands that were produced when examining a complex mixture of antigens, Grabar submitted the antigen mixture to electrophoresis before carrying out the double immune diffusion reaction. This separated the complex series of bands into a set of independent double diffusion reactions at locations imposed by ionization characteristics of antigens (Arquembourg, 1975).

The rate at which antigen and antibody collide in immunoelectrophoresis is greater than during immunodiffusion. The time necessary for the primary reaction between antibody and antigen to develop during immunoelectrophoresis tests is comparable to that of immunodiffusion tests: however, the time necessary for a secondary reactions that lead to lattice formation, complex aggregation, and finally precipitation is not always sufficient, and results in antigen and antibody passing right through each other (Crowle, 1973).

\subsubsection{Buffer}

The effects of constituents of the buffer used for CIE are critical as they modulate the electrochemical properties of the reactants and conditions of the assay itself. In terms of the ionic strength of the buffer it was previously explained that by lowering it temperature is lowered, and fraction migration is increased due to a reduction in endosmotic flow.

However lowering ionic strength also lowers electrophoretic resolution as high ionic strength buffer minimizes interactions between the migrating fractions and the supporting medium (Crowle, 1973). It must also be pointed out in order to lower conductivity of the buffer one must reduce the concentration of its constituent buffering molecules thus reducing the buffering capacity of the buffer. If buffering 
capacity is not sufficient electrophoretic migration of $\mathrm{H}+$ and $\mathrm{OH}$ - ions can result in changes in $\mathrm{pH}$ primarily at the termini of the medium that result in negative effects. For CIE to work the $\mathrm{pH}$, is critical as it dictates the electrophoretic charge of the migrating fractions. The buffer $\mathrm{pH}$ needs to be alkaline enough that the net charge of the antigen becomes electronegative and the net charge of the antibody sera is neutral. However like all components there is an effective range of buffer ph; if the pH is too high it inhibits the precipitin reaction (Verbruggen, 1975).

Commonly used buffer conditions are an ionic strength between 0.02-0.1 M although calculated ionic strength frequently agrees with what is actually determined by electrical conductance (Crowle, 1973) and the most commonly used pH for CIE is between 8.4-8.6 (Arquembourg, 1975; Axelsen, 1973; Crowle, 1973). Also Calciumlactate is often added as its addition results in a higher resolution and clearer band precipitation most likely due to the affinity of many proteins for bi-valent cations such as $\mathrm{Ca}^{2+}$ and $\mathrm{Mg}^{2+}$ (Anhalt \& Yu, 1975; Axelsen, 1973; Verbruggen, 1975). In fact a Tris-tricine based buffer containing Ca-lactate has been shown to work well for CIE (Anhalt \& Yu, 1975).

\subsection{Modifying ENA antigens for CIE}

Traditionally in a CIE assay there is a staining and wash step to improve perception of the precipitin bands. These steps are both a time consuming and labour intensive component of the CIE assay. A novel method to decrease the time required to reach assay completion is to modify specific antigens to fluoresce under a light source. This removes the need to wash and stain the gel achieving the goal of reducing the time required to reach assay completion. A fluorescent dye can be covalently linked to an antigen using one of the commercially available amine reactive dyes. The amine reactive dyes contain a $\mathrm{N}$-hydroxysuccinimide (NHS) ester that undergo a substitution reaction with the amines at the $\mathrm{N}$-terminals and lysine side chains to form an amide bond between the fluorophore of the dye and the protein. This allows realtime observation of the precipitin reaction upon excitation of the fluorophore.

The modification of antigens proposes several problems. The site at which the fluorophore binds to the antigen may be the same as an antibody binding-site, or if 
the location the fluorophore binds is close to that of an antibody binding-site it may physical block the antibody from accessing the binding site. If either of these events occur the antigenicity of the antigen will be reduced. Use of a modified antigen with reduced antigenicity will result in decreased assay sensitivity and will make the modified antigen unsuitable for use in a clinical diagnostic assay. Alternatively, it is possible that binding of the fluorophore to the antigen may increase antigenicity as antibodies may interact with the fluorophore either specifically (binding to the fluorophore) or non-specifically (fluorophore stabilises a weak Ag-Ab interaction). If the antigenicity is increased it will alter the specificity of the assay result and again make the modified antigen unsuitable for use in a clinical diagnostic assay.

\subsubsection{Modification of ENA antigens with Alexa Fluor ${ }^{\circledR} 488$ amine reactive dye} An internal study performed by Arotec Diagnostics (results unpublished) examined the possibility of using fluorophore-labelled ENAs to improve the CIE and DID assay methodology for diagnostic testing. In this study the ENAs were labelled with the Alexa-Fluor® 488 (AF488) amine reactive dye from Invitrogen. The results of this study demonstrated that labelling did not change the chemical properties of the antigen and that the CIE and DID assay methodology for diagnostic testing could be improved by using labelled-ENAs (results not shown). However, the cost of licensing the AF488 dye from Invitrogen restricted the commercial feasibility of using labelledENAs in CIE and DID diagnostic tests. The Dylight 488 (D488) amine reactive dye from Pierce has been identified as an alternative to AF488 for the labelling of ENA antigens. 


\subsection{Aims}

The aims of this project are as follows

1. To develop and optimise a novel CIE assay for the detection of ENA autoantibodies. This is includes comparison and selection one of two amine reactive dyes; Alexa Fluor® 488 (Invitrogen) or Dylight 488 (Pierce). The CIE assay conditions to be optimised include; gel composition, buffer composition, antigen concentration, and the running time of the assay.

2. To evaluate clinically, the optimised novel CIE assay, and an in-house ELISA with a set of patient plasma samples sourced from the Wellington Hospital Immunology Department serum bank and four ENAs SSA, SSB, RNP/Sm, and Sm.

3. To evaluate the effectiveness of the CIE assay compared to that of the ELISA using statistical analysis.

4. To examine this data and provide a critical analysis of the clinical utility of the novel CIE assay for each respective ENA antigen examined. 


\section{Materials and Methods}

\subsection{Antigen and Anti-sera}

All antigens SSA, SSB, RNP/Sm, Sm, Scl-70, and Jo-1 were derived from bovine thymus and produced by Arotec Diagnostics (www.arodia.com); Antigen catalogue numbers SSA \#ATR02-02, SSB \#ATL01-02, RNP/Sm \#ATR01-02, Sm \#ATS02-02, Scl70 \#ATS01-02, and Jo-1 \#ATJ01-02. The anti-RNP sera and anti-SSA/SSB sera used as both positive and negative controls were sourced from The Binding Site (www.bindingsite.com). The anti-Sm, anti Jo-1, and anti-Scl-70 were Arotec Diagnostics internal standards. Patient serum samples used to validate assay specificity were sourced from Wellington Hospital Immunology Department sera bank.

\subsection{Preparation and Labelling of Protein with Dylight-488 Fluor}

Antigen samples for labelling were thawed and dialysed overnight in carbonate coupling buffer $(0.1 \mathrm{M}$ carbonate, $0.4 \mathrm{M} \mathrm{NaCl}, \mathrm{pH}$ 8.8). The protein concentration was determined using a BCA protein assay kit (Pierce).

For protein labelling $1 \mathrm{mg}$ of Dylight 488 (D488) amine-reactive dye (Pierce) was solubilised in $200 \mu \mathrm{L}$ dimethylformamide and then added to the protein sample, and mixed well by vortexing. The reaction proceeded in the dark at room temperature (RT). The reaction was stopped by adding $1 \mathrm{ml}$ of $1 \mathrm{M}$ Tris- $\mathrm{HCl}, \mathrm{pH} 8.0$. The excess D488 was removed using a PD-10 desalting column (GE Life Sciences) according to the Manufacturer's instructions. The wash buffer used was $20 \mathrm{mM}$ Tris $\mathrm{HCl},(\mathrm{pH} 7.5)$, $0.4 \mathrm{M} \mathrm{NaCl}, 3.5 \mathrm{mM} \mathrm{MgCl}_{2}$.

\subsection{Antigenicity of Labelled vs Unlabelled Proteins}

The antigens SSA, SSB, RNP/Sm, Sm, Jo-1, and Scl-70 were labelled with D488 fluor at ratios (protein:fluor) of 640:1, 320:1, 160:1, 80:1, 40:1, 20:1, 10:1, and 5:1. The D488-labelled proteins were examined by sodium dodecyl sulphate polyacrylamide gel electrophoresis (SDS-PAGE) visualised with using blue light

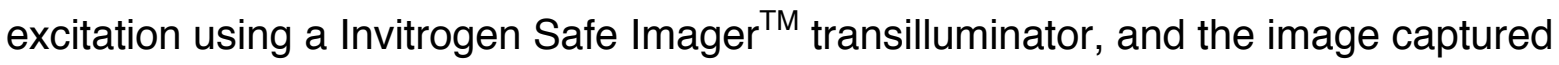


using a Gel Doc ${ }^{\mathrm{TM}} \mathrm{XR}$ (Bio-Rad). The gels were fixed and stained with Coomassie blue and an image taken using white light transillumination in the Gel $\mathrm{Doc}^{\mathrm{TM}} \mathrm{XR}$ (BioRad). Images were analysed using Quantity One 1-D (Bio-Rad) software.

Duplicates of SDS-PAGE gels were also examined by Western blotting. Gel proteins were transferred to nitrocellulose and placed into Western blot blocking buffer (10 $\mathrm{mM}$ phosphate, $(\mathrm{pH} 7.4), 0.15 \mathrm{M} \mathrm{NaCl}, 0.02 \% \mathrm{w} / \mathrm{v}$ Tween $20,0.5 \% \mathrm{w} / \mathrm{v}$ bovine serum albumin (BSA), $0.1 \% \mathrm{w} / \mathrm{v}$ Bronidox $\mathrm{L}$ ) for $1 \mathrm{~h}$. The blocking buffer was poured off and $6 \mathrm{ml}$ Western blot antibody dilution buffer (10 mM phosphate $(\mathrm{pH} 7.4), 0.15 \mathrm{M} \mathrm{NaCl}$, $0.02 \% \mathrm{w} / \mathrm{v}$ Tween $20,1 \% \mathrm{w} / \mathrm{v}$ sodium caseinate, $0.1 \% \mathrm{w} / \mathrm{v}$ Bronidox $\mathrm{L}$ ) was added. For each respective antigen $20 \mu \mathrm{l}$ of its corresponding anti-sera was pipetted into the Western blot antibody dilution buffer to give a sera dilution of 1:300. Blots were rocked overnight at RT. The nitrocellulose blots were washed 3 times in phosphate buffered saline solution with Tween 20 (PBST) wash buffer (10 mM phosphate (pH 7.4), $0.15 \mathrm{M} \mathrm{NaCl}, 0.02 \% \mathrm{w} / \mathrm{v}$ Tween $20,0.1 \% \mathrm{w} / \mathrm{v}$ Bronidox L). Anti-human alkalinephosphatase antibody conjugate (Jackson) was diluted 1:6000 in Western blot antibody dilution buffer and $6 \mathrm{ml}$ pipetted onto each blot. Blots were incubated for $1 \mathrm{~h}$ at RT. Blots were washed 3 times in PBST wash buffer. The blots were developed in $5 \mathrm{ml}$ alkaline phosphatase development buffer (50 mM Tris-HCL (pH 9.0), $100 \mathrm{mM}$ $\mathrm{NaCl}, 5 \mathrm{mM} \mathrm{MgCl}$ ) and $50 \mu \mathrm{l}$ alkaline phosphatase substrate (25 mM nitro-blue tetrazolium chloride, $22 \mathrm{mM}$ 5-bromo-4-chloro-3'-indolyphosphate p-toluidine salt, $70 \% \mathrm{w} / \mathrm{v}$ dimethyl sulfoxide). The reaction was stopped by washing blots with deionised $\mathrm{H}_{2} \mathrm{O}$. Images were taken using the Gel Doc ${ }^{\top \mathrm{M}}$ XR (Bio-rad).

Both unlabelled and D488-labelled antigens labelled at a ratio of 5:1 were analysed by enzyme linked immunoabsorbant assay (ELISA). For each antigen two ELISA assays were performed at two different antigen coating concentrations. For each 96well NUNC Maxisorp ELISA plate, half (48 wells) was coated with $0.1 \mathrm{ml}$ unlabelled antigen in coating buffer ( $20 \mathrm{mM}$ Tris- $\mathrm{HCl},(\mathrm{pH} 8.0), 0.15 \mathrm{M} \mathrm{NaCl})$ and the other half coated with its equivalent D488-labelled antigen. The two plating concentrations for SSA were $1 \mu \mathrm{g} / \mathrm{ml}$ and $0.5 \mu \mathrm{g} / \mathrm{ml}$; for SSB $0.5 \mu \mathrm{g} / \mathrm{ml}$ and $0.25 \mu \mathrm{g} / \mathrm{ml}$; for RNP $/ S \mathrm{~m} 0.5$ $\mu \mathrm{g} / \mathrm{ml}$ and $0.25 \mu \mathrm{g} / \mathrm{ml}$; for Sm $0.5 \mu \mathrm{g} / \mathrm{ml}$ and $0.25 \mu \mathrm{g} / \mathrm{ml}$. Plates were blocked for $3 \mathrm{~h}$ at RT with coating buffer, containing $0.1 \% \mathrm{w} / \mathrm{v}$ BSA. The ELISA plates were washed 3 times with wash buffer ( $20 \mathrm{mM}$ Tris- $\mathrm{HCl},(\mathrm{pH} 7.5), 0.3 \mathrm{M} \mathrm{NaCl}, 0.1 \%$ Tween 20$)$. Then each half plate was then incubated with 23 antigen positive sera samples ( 0.1 
$\mathrm{ml}$ ) and 1 negative control in duplicate $(0.1 \mathrm{ml})$, both at a 1:100 dilution in antibody dilution buffer (20 mM Tris- $\mathrm{HCl},(\mathrm{pH}$ 7.5), 0.3M NaCl, 0.1\% Tween 20, 0.1\% w/v BSA, and $0.1 \%$ sodium caseinate). The ELISA plates were left at RT for $1 \mathrm{~h}$ and then washed 3 times with wash buffer. Then $0.1 \mathrm{ml}$ of horse radish peroxidase-conjugated goat anti-human IgG (Jackson ImmunoResearch Laboratories) at 1:30,000 dilution in antibody dilution buffer was added per well and incubated for $1 \mathrm{~h}$ at RT. Plates were washed 3 times in wash buffer and developed in $0.15 \mathrm{ml}$ of developer solution (0.1 M sodium acetate, $(\mathrm{pH} 5.5), 0.0125 \% \mathrm{w} / \mathrm{v}$ tetramethylbenzidine, $0.03 \% \mathrm{w} / \mathrm{v}$ hydrogen peroxide). The reaction was stopped by the addition of $50 \mu \mathrm{l}$ of $2 \mathrm{M} \mathrm{H}_{2} \mathrm{SO}_{4}$ per well. Plates were read at $450 \mathrm{~nm}$ using a Biotek Powerwave 340 plate reader.

\subsection{Fluorecent Intensity of Labelled Protein}

Two fold serial dilutions were prepared (range $0.0313 \mu \mathrm{g}, 0.0625 \mu \mathrm{g}, 0.125 \mu \mathrm{g}, 0.25$ $\mu \mathrm{g}, 0.5 \mu \mathrm{g}, 1.0 \mu \mathrm{g}$, and $2.0 \mu \mathrm{g}$ ) of the proteins SSA, and RNP/Sm labelled with D488 at a 5:1 ratio and examined by SDS-PAGE. The gel was viewed through an amber

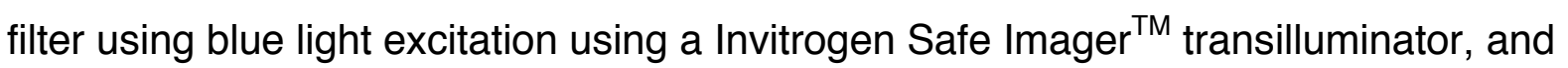
the image captured using a Gel Doc ${ }^{\mathrm{TM}} \mathrm{XR}$ (Bio-Rad). The gels were then fixed and stained with Coomassie blue and an image taken using white light transillumination in the Gel Doc ${ }^{T M}$ XR (Bio-Rad). Images were analysed using Quantity One 1-D (BioRad) software. 


\subsection{CIE Apparatus modifications and configurations}

A number of prototypes of the CIE apparatus were trialled:

\subsubsection{Apparatus 1}

An Amersham Biosciences powerpack connected to the modified insert of the Invitrogen $\mathrm{iBase}^{\mathrm{TM}}$ illuminator by two lead cables. Electrophoresis with apparatus 1 uses constant current and variable voltage.

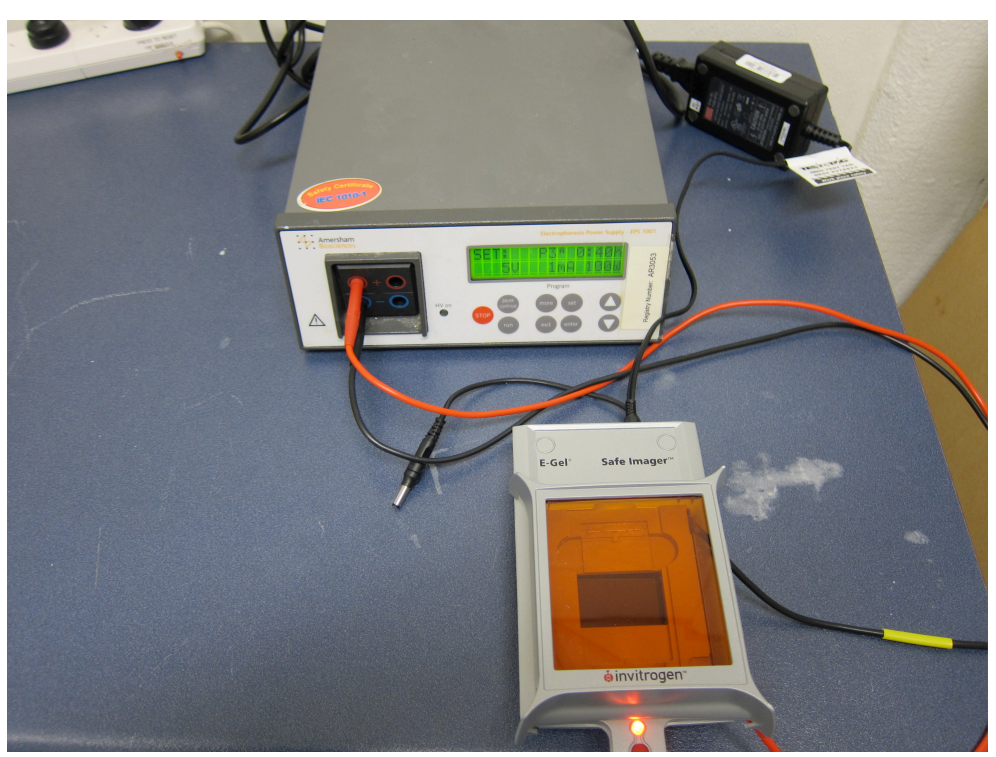

Figure 2 Apparatus 1 - cover on

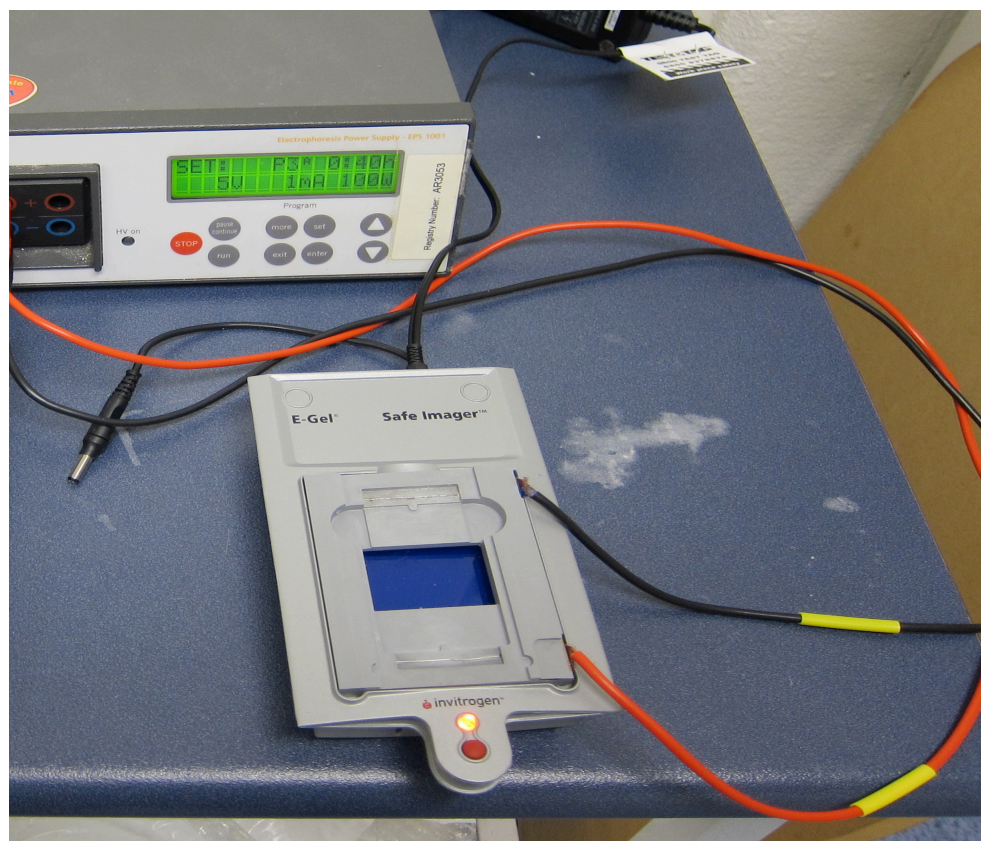

Figure 3 Apparatus 1 - cover off 


\subsubsection{Apparatus 2 (modified ibase)}

The modified insert in apparatus 1 was inserted into a modified iBase ${ }^{\mathrm{TM}}$ unit that has an external resistor that can be used to restrict current. Electrophoresis with apparatus 2 uses variable current at constant voltage.

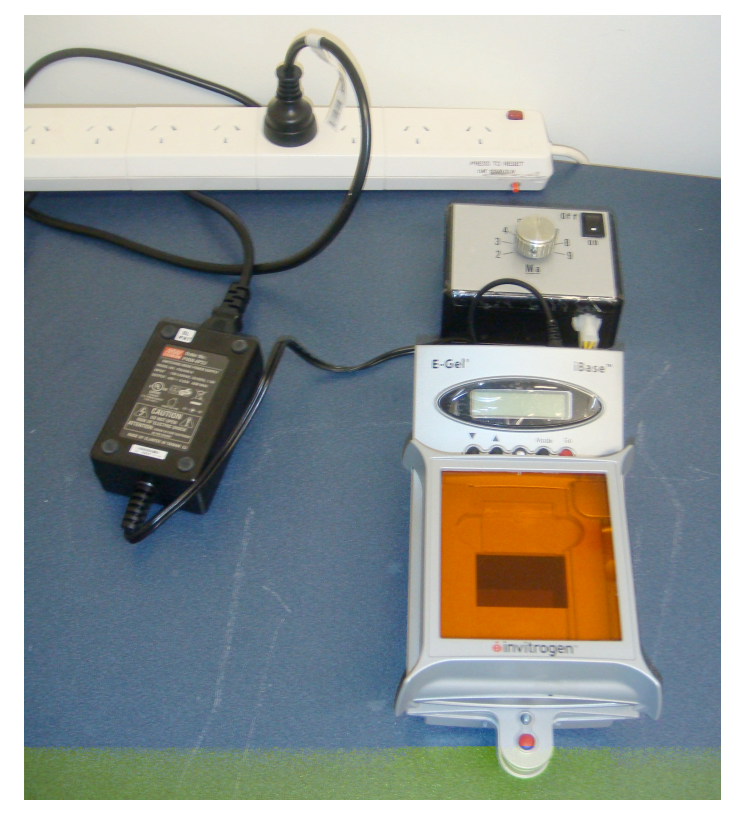

Figure 4 Apparatus 2

\subsubsection{Apparatus 3 (unmodified ibase)}

An unmodified ibase unit was used in conjunction with a modified $\mathrm{iBase}^{\mathrm{TM}}$ insert that had an internal resistor to restrict current to $5 \mathrm{~mA}$. Electrophoresis with apparatus 3 uses variable current at constant voltage.

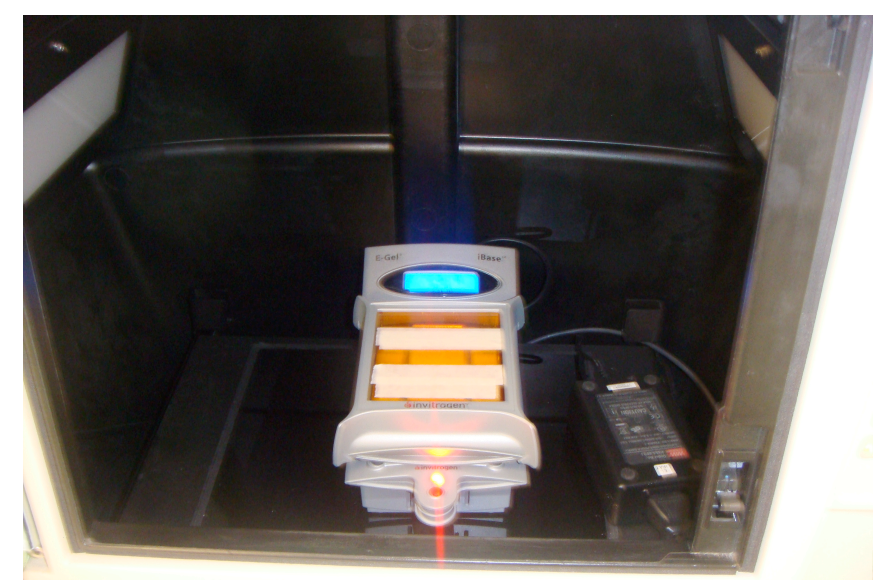

Figure 5 Apparatus 3 inside Gel Doc XR 


\subsection{Comparison of Dylight 488 vs Alexa Fluor® 488}

\subsubsection{Preparation and labelling of protein with Alexa Fluor ${ }^{\circledR} 488$}

Preparation of and labelling of the antigens SSA, SSB, RNP/Sm, and Sm with Alexa Fluor® 488 (Invitrogen) was performed by a colleague as per the Manufacturer's instructions. Protein concentration was determined using a BCA protein assay kit (Pierce).

\subsubsection{Comparison of fluorescent intensity D488 vs AF488}

The D488-labelled and AF488-labelled antigens (SSA, SSB, RNP/Sm, and Sm) were examined by SDS-PAGE. The gels were visualised using blue light excitation using a Invitrogen Safe Imager ${ }^{\mathrm{TM}}$ transilluminator, and the image captured using a Gel Doc ${ }^{\mathrm{TM}}$ XR (Bio-Rad). The same gels were fixed and stained with Coomassie blue and an image captured using white light transillumination in the Gel Doc ${ }^{T M}$ XR (Bio-Rad). Images were analysed using Quantity One 1-D (Bio-Rad) software.

\subsubsection{Comparison of D488 vs AF488 by CIE}

The D488-labelled and AF488-labelled antigens (SSA, SSB, RNP/Sm, and Sm) were examined by CIE. Each CIE assay was loaded in duplicate with a set of serum samples; one positive sera control, one negative sera control, and a clinical sample from the Wellington Hospital Immunology department. One set of sera was run against D488-labelled antigen and the other against AF488-labelled antigen. CIE assays were performed with Apparatus 3 and the standard CIE method (see below). 


\subsection{Optimisation of Counter Immunoelectrophoresis}

\subsubsection{Standard Counter Immunoelectrophoresis Method}

A single agarose gel was placed onto the iBase ${ }^{\mathrm{TM}}$ insert. A $2.5 \times 35 \times 30 \mathrm{~mm}$ (heightdepth-width) paper strip from Pall was placed on the iBase $^{\mathrm{TM}}$ insert overlapping the cathode terminal wire and the upper portion of the gel to act as a wick. A second wick of the same dimensions was placed on the iBase ${ }^{\mathrm{TM}}$ insert overlapping the anode terminal wire and the lower portion of the gel (Fig. 6). A $2.5 \mathrm{~mL}$ aliquot of buffer (see recipe below) was then pipetted onto each paper strip. Sera $(10 \mu \mathrm{l})$ was then pipetted into each of lower wells (anodic side) of the gel. A pre-electrophoresis step was then performed by applying voltage to the gel before the addition of antigen for $5 \mathrm{~min}$. After pre-electrophoresis, $5 \mu \mathrm{l}$ of antigen was pipetted into the upper cathodic side wells of the gel. Electrophoresis was then performed on the gel until the assay endpoint was reached. The assay endpoint was determined to be the point the antigen in the negative control passes into the anodic antibody wells. The time required to reach the assay was antigen dependent. For SSA and SSB the time required was 30 min electrophoresis, $\mathrm{Sm}$ required $50 \mathrm{~min}$, and RNP/Sm required 60 min. Images of gels were acquired using the Gel Doc ${ }^{T M}$ XR (Bio-Rad).

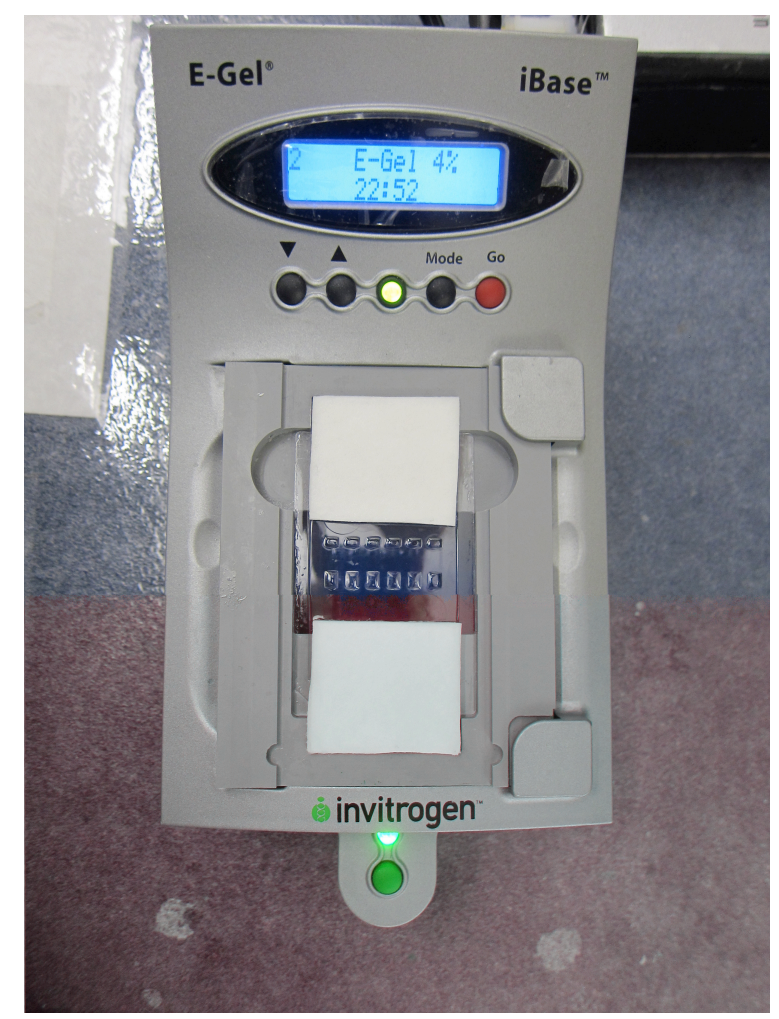

Figure 6 CIE Apparatus 2 cover off - Wick and gel placement 


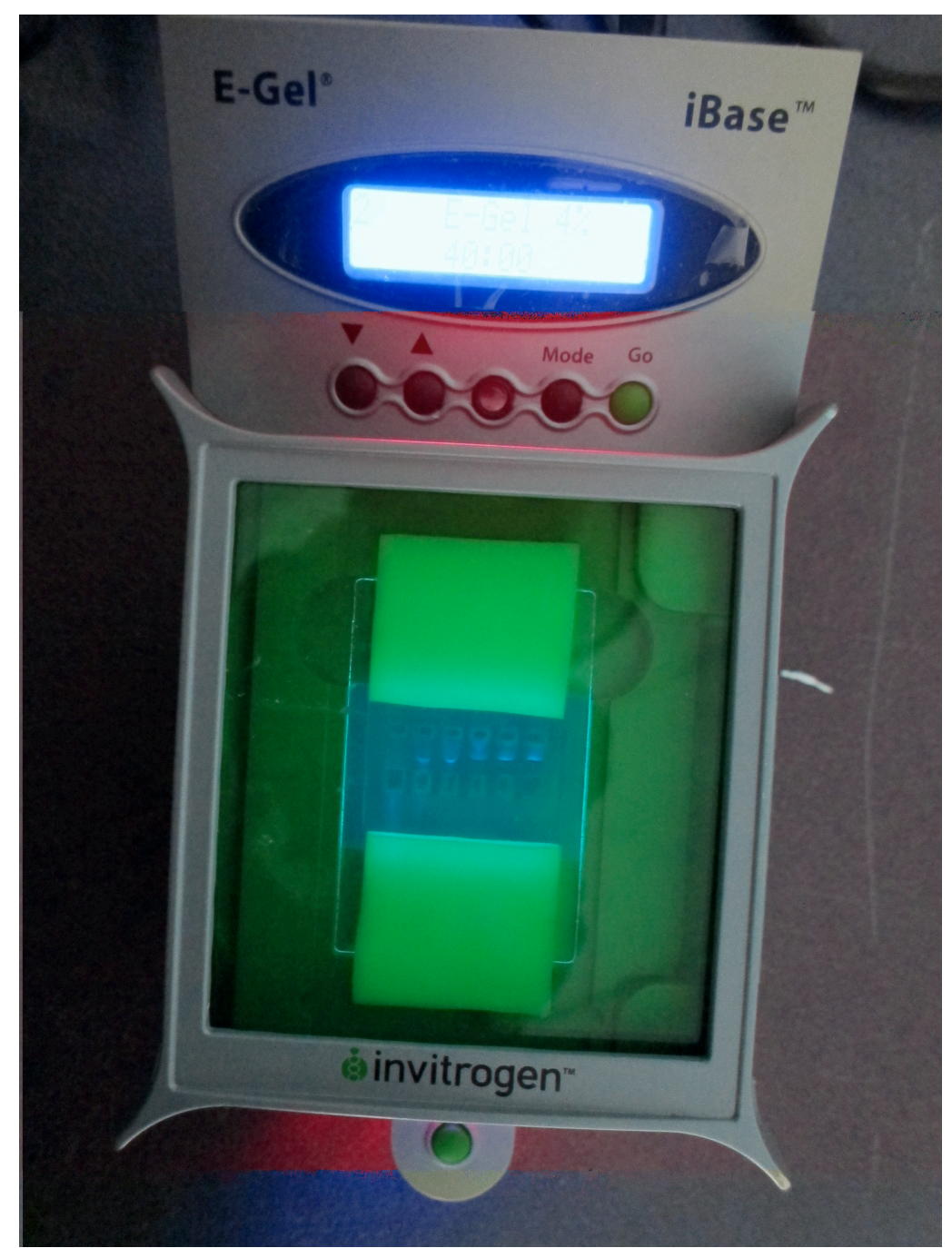

Figure 7 CIE Apparatus 2 cover on - assay running

\subsubsection{Agarose Gels}

High electro-endosmosis (HEEO) agarose (Roth) was dissolved in buffer (see recipe below) by heating in a microwave until it reached boiling point and then swirled gently until dissolved. Once dissolved, $2.5 \mathrm{~mL}$ of dissolved agarose solution was pipetted into a gel mould and a Gelbond® plastic film (Lonza) was placed over the surface of the mould. Gels were left at RT for 30 min to set. Each gel was then placed into a sealed plastic bag and stored at $4^{\circ} \mathrm{C}$ until use.

\subsubsection{Agarose concentration and Current}

Experiments were undertaken to determine the effect of agarose concentration, and the current applied during electrophoresis on CIE results.

Using apparatus $1 \mathrm{CIE}$ assays were run at constant current of $3 \mathrm{~mA}, 5 \mathrm{~mA}$, or $7 \mathrm{~mA}$. Agarose gels were made at $0.4 \%, 0.6 \%, 0.8 \%, 1.0 \%$, and $1.2 \% \mathrm{w} / \mathrm{v}$ agarose 
concentrations in buffer (80 mM Tris, (pH 8.6), 24 mM Tricine). For each current, a gel was run at a different agarose concentration under the following conditions; 5 min pre-electrophoresis, and electrophoresis $60 \mathrm{~min}$ at $3 \mathrm{~mA}, 40 \mathrm{~min}$ at $5 \mathrm{~mA}, 20 \mathrm{~min}$ at 7 $\mathrm{mA}$, and contained SSA positive and negative control sera, and D488-labelled SSA antigen.

\subsubsection{Pre-electrophoresis time}

Using apparatus 1, two separate CIE assays were run with the positive and negative serum controls loaded at staggered time intervals of $5 \mathrm{~min}$. For CIE assay 1, the time intervals were 5, 10, and 15 min pre-electrophoresis. For CIE assay 2, the time intervals were $0,5,10,15$, and 20 min pre-electrophoresis. After the last preelectrophoresis interval D488 labelled SSA antigen was then loaded and the CIE assay run for another $40 \mathrm{~min}$.

\subsection{Buffer}

\subsubsection{Calcium lactate titration}

A series of CIE buffers were made ( $80 \mathrm{mM}$ Tris, $24 \mathrm{mM}$ Tricine, $0.05 \% \mathrm{w} / \mathrm{v} \mathrm{NaN}_{3}$ ) with a titration (range $1 \mathrm{mM}, 2 \mathrm{mM}, 5 \mathrm{mM}, 10 \mathrm{mM}, 20 \mathrm{mM}$, and $40 \mathrm{mM}$ ) of Calciumlactate. Agarose gels $(0.6 \% \mathrm{w} / \mathrm{v})$ were made using each of the buffers. CIE assays of four 2-fold sera dilution series of the serum positive control, and a single serum negative control were performed under the following conditions; Apparatus 2, at 5 $\mathrm{mA}, 5 \mathrm{~min}$ pre-electrophoresis, and 45 min electrophoresis. 


\subsubsection{Buffer pH}

A range of buffers containing varying concentrations of Tris and Tricine, and a different $\mathrm{pH}$ were examined for their effect on CIE. Tris and Tricine concentrations were altered antagonistically in order to keep conductivity relatively the equal between buffers.

Buffer 1; $60 \mathrm{mM}$ Tris, $32 \mathrm{mM}$ Tricine, $5 \mathrm{mM}$ Calcium-lactate, 0.05\% w/v $\mathrm{NaN}_{3}$, (pH 8.4), conductivity: $0.19 \mu \mathrm{M} / \mathrm{s}$.

Buffer 2; $80 \mathrm{mM}$ Tris, $28 \mathrm{mM}$ Tricine, $5 \mathrm{mM}$ Calcium-lactate, 0.05\% w/v $\mathrm{NaN}_{3}$, (pH 8.6), conductivity: $0.19 \mu \mathrm{M} / \mathrm{s}$.

Buffer 3; 90 mM Tris, 20 mM Tricine, 5 mM Calcium-lactate, 0.05\% w/v NaN $\mathrm{N}_{3}$, (pH 8.8), conductivity: $0.2 \mu \mathrm{M} / \mathrm{s}$.

The buffers were heated using a Heidolph Magnetic Stirrer MR Hei-Standard and the temperature and $\mathrm{pH}$ measured using the Orion $4 \mathrm{Star} \mathrm{pH}$ and conductivity meter. Agarose gels $(0.6 \% \mathrm{w} / \mathrm{v})$ were made using each of the buffers. CIE assays were performed using Apparatus 2, at $5 \mathrm{~mA}$, with $5 \mathrm{~min}$ pre-electrophoresis, and $45 \mathrm{~min}$ electrophoresis.

\subsubsection{Tris-Tricine concentration}

Three buffers were made up with three different concentrations of Tris and Tricine. The ratio of Tris and Tricine were the same for each buffer to keep $\mathrm{pH}$ the same but altering conductivity between buffers.

Buffer 1A ; $45 \mathrm{mM}$ Tris, $10 \mathrm{mM}$ Tricine, $5 \mathrm{mM} \mathrm{Ca-lactate,} \mathrm{0.05 \%} \mathrm{w/v} \mathrm{NaN}$, (pH 8.8), conductivity: $0.15 \mu \mathrm{M} / \mathrm{s}$.

Buffer 2A ; $90 \mathrm{mM}$ Tris, $20 \mathrm{mM}$ Tricine, $5 \mathrm{mM}$ Ca-lactate, 0.05\% w/v NaN (pH 8.8), conductivity: $0.2 \mu \mathrm{M} / \mathrm{s}$.

Buffer 3A ; $180 \mathrm{mM}$ Tris, $40 \mathrm{mM}$ Tricine, $5 \mathrm{mM}$ Ca-lactate, 0.05\% w/v NaN (pH 8.8), conductivity: $0.4 \mu \mathrm{M} / \mathrm{s}$

Agarose gels $(0.6 \% \mathrm{w} / \mathrm{v})$ were made and CIE assays performed under the following assay conditions; Apparatus 2, at $5 \mathrm{~mA}$, as per the standard method. 


\subsection{Antigen Concentrations}

Serial 2-fold dilutions of the D488-labelled antigens SSA, SSB, RNP/Sm, and Sm were made (range $10 \mathrm{ng} / \mu \mathrm{l}$ to $80 \mathrm{ng} / \mu \mathrm{l}$ ). A CIE assay was performed for each antigen with a positive sera control for each antigen concentration. Also a negative sera control was also run against $10 \mathrm{ng} / \mu \mathrm{l}$ and $80 \mathrm{ng} / \mu \mathrm{l}$. Assay conditions used: Apparatus 2 at $5 \mathrm{~mA}$, and the standard CIE method.

\subsection{Antigen Concentration and Analytical Sensitivity}

Three 2-fold sera dilution series (range 1/2 to 1/256) were created using SSA/SSB, $\mathrm{RNP} / \mathrm{Sm}$, and Sm positive sera and unreactive sera. Using the respective dilution series 3 sets of CIE assays were performed using SSA antigen at concentrations 10, 20 , and $40 \mathrm{ng} / \mu \mathrm{l}$. Using the respective dilution series, 2 sets of CIE assays were performed for the SSB, RNP/Sm and Sm antigens at concentrations of 20, and 40 $\mathrm{ng} / \mu \mathrm{l}$. All CIE assays were performed using Apparatus 3 and the standard $\mathrm{CIE}$ method.

\subsection{Analytical Specificity}

Tests were undertaken to examine the analytical specificity of the method. Four CIE assays were performed, each with a different D488-labelled antigen (SSA, SSB, $\mathrm{RNP} / \mathrm{Sm}$, or $\mathrm{Sm}$ ). For each assay the following 6 positive control sera samples were run for SSA, SSA/SSB, RNP, Sm, Jo-1, and Scl-70 and performed using Apparatus 3 and the standard CIE method.

\subsection{CIE Time Series}

Tests were undertaken to determine the optimum length of time for a differential positive $\mathrm{CIE}$ test for each antigen. The end point of the assay (assay completion) is when the antigen runs through the gel past the negative control sera well. SSA, SSB, $\mathrm{RNP} / \mathrm{Sm}$, and $\mathrm{Sm}$ were run against their respective positive sera control, a negative sera control, and a buffer blank. Assays were performed using Apparatus 3 and the standard CIE method. Apparatus 3 was placed inside the Gel Doc ${ }^{\mathrm{TM}}$ XR (Bio-Rad) 
during the run. Blue-light excitation from the iBase ${ }^{\mathrm{TM}}$ (Invitrogen) was used to capture images periodically throughout each run without stopping the run.

\subsection{Clinical Validation of CIE Assays}

For each of the following antigens SSA, SSB, RNP/Sm, and Sm CIE and ELISA assays were performed using 281 ANA IIF positive clinical patient serum samples sourced from the Wellington Hospital Immunology Department serum bank.

Our methods for review of the clinical data were forwarded to the Central Regional Ethics Committee who advised formal ethical approval was not required under two exemptions of the Ethical Guidelines for Observational Studies: Observational Research, Audits and Related Activities, NEAC, December 2006. No further review was required.

\subsubsection{CIE assay}

Each CIE assay was run with a positive and negative control serum, and four patient sera samples. Where less than four patient sera samples were run, buffer was loaded into the serum well instead. CIE assays were scored with a 0,1 , or 2 at the endpoint of the assay; 0 conclusive negative result; 1 inconclusive result; 2 positive result with clear precipitation reaction. Results were re-scored by a colleague familiar with the CIE assay, who was blinded to the sample identity to confirm results.

For the purpose of CIE evaluation scores 0 and 1 were considered to analytically negative and score 2 were analytically positive. CIE assays performed using apparatus 3 and the standard CIE method. 


\subsubsection{ELISA assay}

The ELISA method was the same as previously described with the following modifications to the coating steps. A 96-well NUNC maxisorp ELISA plates was coated with $100 \mu \mathrm{l}$ of each of the four antigens at a concentration of $1 \mu \mathrm{g} / \mathrm{ml}$ diluted in coating buffer. After blocking, each plate was incubated with $100 \mu \mathrm{l}$ of the Wellington Hospital Immunology Department serum bank sera samples, 4 positive controls and 1 negative control all diluted 1:100 in antigen dilution buffer.

The endpoint of the ELISA assay was determined for each antigen (SSA, SSB, RNP/Sm, and Sm) using the formula and method described by Frey et al (1998). The ELISA was performed as previously described with the following modifications. Each of the four antigens was used to coat a quarter of a 96-well NUNC Maxisorp ELISA plate: plates were coated with $100 \mu$ of antigen at a concentration of $1 \mu \mathrm{g} / \mathrm{ml}$ diluted in coating buffer. After blocking, each antigen sector was incubated with $100 \mu$ from 22 respective negative control sera $(n=22)$ and 1 positive control serum diluted 1:100 in antigen dilution buffer, and also an antibody dilution buffer blank. The negative and positive control sera were Arotec internal standards. The $t$-value $(t=$ 2.518) was determined from a one-tailed $t$-distribution table with $99 \%$ confidence interval and 21 degrees of freedom. 


\subsubsection{Clinical data and classification}

Categorical clinical data was available for 156/281 serum samples. The categorical data came from a previous study (unpublished) performed at Wellington Hospital. Categorisation was performed by a clinical immunology registrar. Electronic patient records were used to assign categories based on the diagnostic criteria for Sjorgren's syndrome and other anti-SSA associated diseases. Each serum sample was given a categorical score based on the following classifications.

1. Primary Sjogren's or subacute cutaneous / neonatal lupus

2. Secondary Sjogren's

3. Probable Primary Sjogren's or subacute cutaneous / neonatal lupus

4. Probable Secondary Sjogren's

5. A definite connective tissue disease but no suggestion of Sjogren's or subacute cutaneous / neonatal lupus

6. Possible Sjogrens or connective tissue disease but information too limited

7. Nil diagnoses and / or lack of evidence for any Sjogren's, subacute cutaneous / neonatal lupus or other connective tissue disease

\subsubsection{Statistical methods}

Data was collated into $2 \times 2$ tables and Cohen's kappa analysis was performed to compare the inter-rata agreement between the CIE and ELISA results. Areaproportional Venn diagrams were generated the BIOINFOX Venn diagram program (http://bioinforx.com/lims/bxtoolbox/area-proportional-venn-diagram-plotter-editordata-extraction-overlapping)

For the serum samples with categorical data available CIE and ELISA results were grouped together into case groups. Case group 1 (categorical groupings consistent with SS) consisted of samples categorised 1-4 $(n=24)$; Case group 2 (categorical grouping consistent with other CTD) consisted of samples categorised 5 and $6(n=$ 40); Case group 3 (control group) consisted of samples in category $7(n=92)$. Data was collated into $2 \times 2$ tables and the diagnostic sensitivity and specificity, as well as the likelihood ratios of the CIE and ELISA assays were calculated. 


\subsubsection{Western blot}

The clinical sera samples were examined by Western blot for each of the following antigens SSA, SSB, RNP/Sm, Sm. SDS-PAGE was performed with $2 \mu \mathrm{g}$ protein per lane for each respective antigen and transferred to nitrocellulose by Western blot. The Western blot method and reagents were the same as previously described with the following modifications. The nitrocellulose blots were divided into strips and blocked individually with blocking buffer. Sera were diluted 1:100 in sera dilution buffer. Each strip was coated in $1 \mathrm{ml}$ of a single sera dilution and incubated overnight at RT. Strips were washed 3 times in PBST wash buffer and coated in alkaline phosphatase conjugated anti-human IgG antibody diluted 1:6000 in antibody dilution buffer. Strips were incubated at RT for $1 \mathrm{~h}$ and then washed 3 times with PBST buffer. Strips were developed in $1 \mathrm{ml}$ of alkaline phosphatase development buffer with $5 \mu$ l of substrate. The reaction was stopped by washing strips in deionized $-\mathrm{H}_{2} \mathrm{O}$.

Each blot was scored 0 for a negative result as compared to the negative control, 2 for a positive result as compared to the positive control, or 1 for an inclusive result as compared to the positive and negative controls.

\subsection{Double Immunodiffusion}

Agarose gels ( $1 \%$ ) were made with high electro-endosmosis (HEEO) agarose (Roth). The agarose was dissolved in buffer (20 mM Tris HC, $(\mathrm{pH} 7.5), 0.4 \mathrm{M} \mathrm{NaCl}$, $3.5 \mathrm{mM} \mathrm{MgCl}_{2}, 5 \mathrm{mM}$ Calcium lactate) by heating in a microwave until it reached boiling point and then swirled gently until dissolved. Once dissolved $0.85 \mathrm{~mL}$ of dissolved agarose solution was pipetted into a gel mould and left to set for $1 \mathrm{hr}$ at RT. Seven wells were then punched out of the agarose.

Pipetted into the centre well, was $5 \mu \mathrm{l}$ RNP/Sm antigen at a concentration of $50 \mathrm{ng} / \mu \mathrm{l}$. This was allowed to diffuse for 10 min before the sera was pipetted into the surrounding wells. Into the surrounding wells $5 \mu \mathrm{l}$ of sera was pipetted. Each gel was loaded with a positive and negative control serum, and four sera samples. In the case that less than four patient sera samples were run, buffer was loaded into the serum well instead. A strip of cellotape was placed over the gel to prevent evaporation of the gel. Gels were left in the dark at RT temperature overnight. The cellotape was 
removed and the gels examined by blue light excitation using a Invitrogen Safe Imager $^{\mathrm{TM}}$ transilluminator, and the image captured using a Gel Doc ${ }^{\mathrm{TM}} \mathrm{XR}$ (Bio-Rad). 


\section{Results}

\subsection{Preparation and Labelling of Protein with Dylight 488 Fluor}

The four antigens Scl-70, RNP/Sm, SSB, and SSA were incubated in series with the

D488 and the labelling reaction stopped at different time intervals. The labelled antigens were examined by blue-light excitation. Fig. 8 shows as the reaction time increases so does the fluorescent intensity of the labelled antigen. For all four antigens there is no further increase in fluorescent intensity after 10 min reaction time.

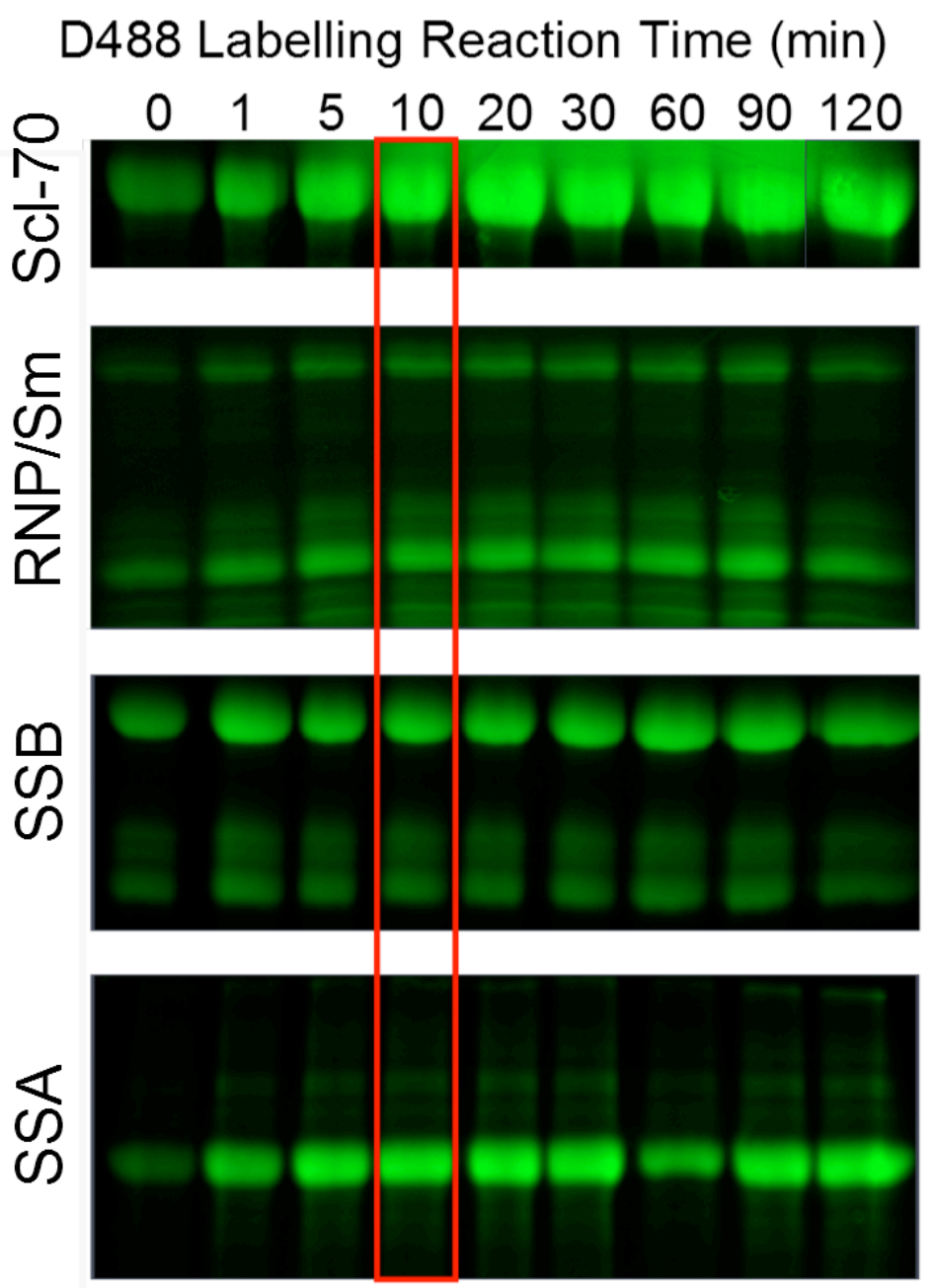

\section{Figure 8 Dylight 488 labelling of ENA proteins reaction time}

Multiple samples of the SSA, SSB, RNP/Sm, Sm antigens were incubated with NHS ester Dylight 488 dye. Reactions were stopped at 0, 1, 5, 10, 15, 20, 30, 60,90 , and $120 \mathrm{~min}$. Samples were examined by SDS-PAGE and visualized through an amber filter with blue-light excitation. Reaction at 10 min highlighted in red. Banding patterns are typical for proteins examined by SDS-PAGE. Multiple bands are present due to dissociation of protein subunits. 


\subsection{Antigenicity of Labelled vs Unlabelled Protein}

The antigens Sm, RNP/Sm, Scl-70, Jo-1, SSA, and SSB were labelled with D488 at ratios (protein:fluor) of $640: 1,320: 1,160: 1,80: 1,40: 1,20: 1,10: 1$, and 5:1. Using the labelling series for each of the six antigens SDS-PAGE was performed and the gels examined by blue-light excitation, Coomassie staining, and Western blot (Fig. 9). As the labelling ratio increases from 640:1 to 5:1 a linear increase in fluorescent intensity was observed. The maximal fluorescent intensity achieved corresponded with the highest labelling ratio of $5: 1$, while no fluorescence was observed at a labelling ratio of 640:1. The Coomassie stained gels (Fig. 9) confirm the consistent loading of total protein per well. The western blots for each antigen show equal binding as the labelling ratio increases from 640:1 to 5:1 indicating that D488 does not prevent antibody binding on Western blot.

Unlabelled and D488-labelled antigens (RNP/Sm, Sm, SSA, and SSB) labelled at a ratio of 5:1 were analysed by ELISA to determine whether the label disrupted antibody binding under native conditions. For each antigen two ELISA assays were performed at two different antigen coating concentrations. When the ELISA data of unlabeled and labelled protein was plotted against each other (Figs 10-13) and linear regression analysis performed, a linear relationship was observed between unlabelled and labelled antigens. Furthermore, for each plot the slope of the trendline tends towards 1, indicating that the label did not decrease or increase the ELISA score in a linear fashion. 


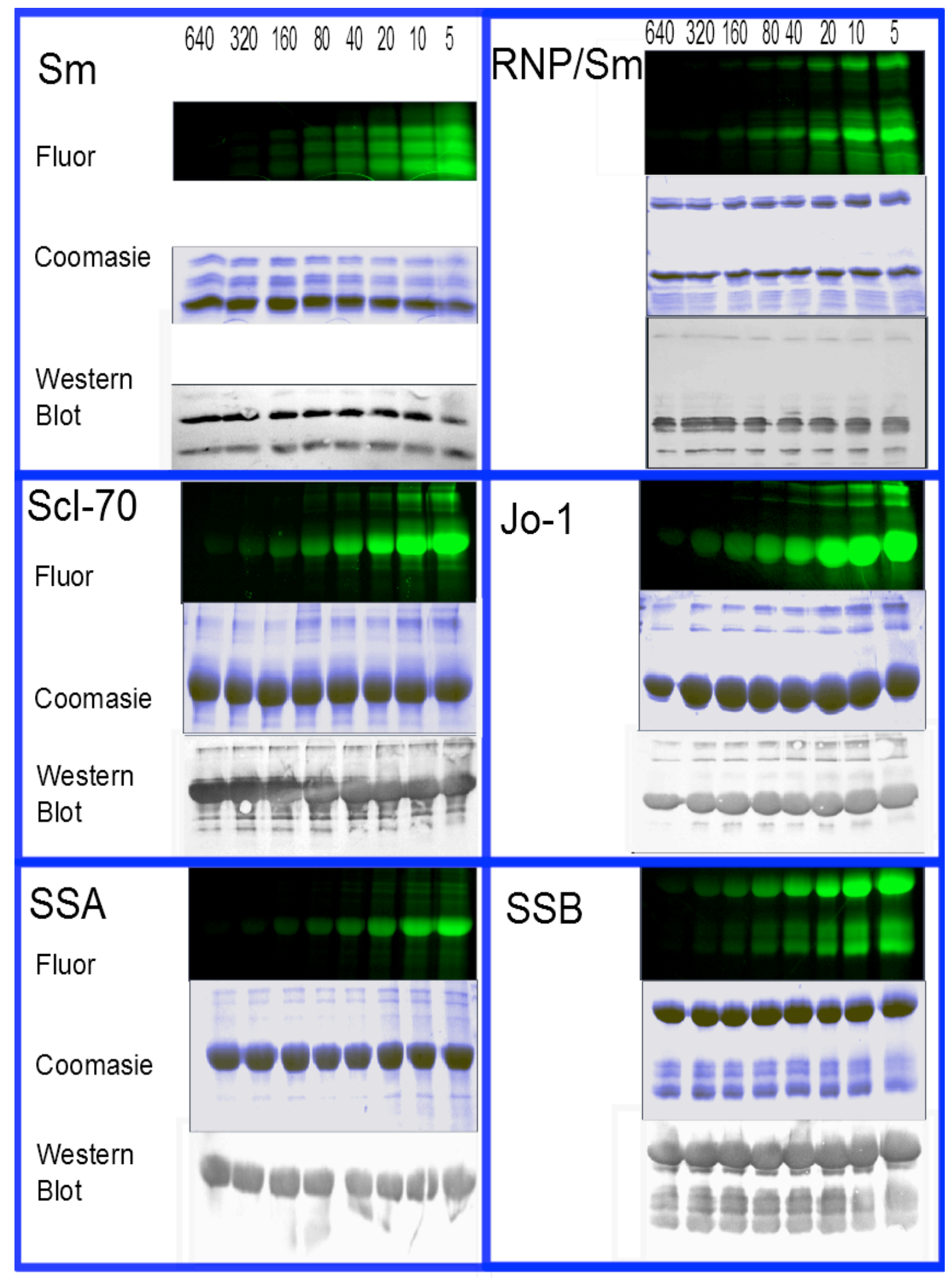

Figure 9 Comparison of antigen labelling

The antigens Sm, RNP/Sm, Scl-70, Jo-1, SSA, and SSB were labelled with D488 at ratios (protein:fluor) of 640:1, 320:1, 160:1, 80:1, 40:1, 20:1, 10:1, and $5: 1$. Using the labelling series for each of the six antigens SDS-PAGE was performed and the gels examined by blue-light excitation (Fluor) using an amber filter, Coomassie staining, and Western blot. 


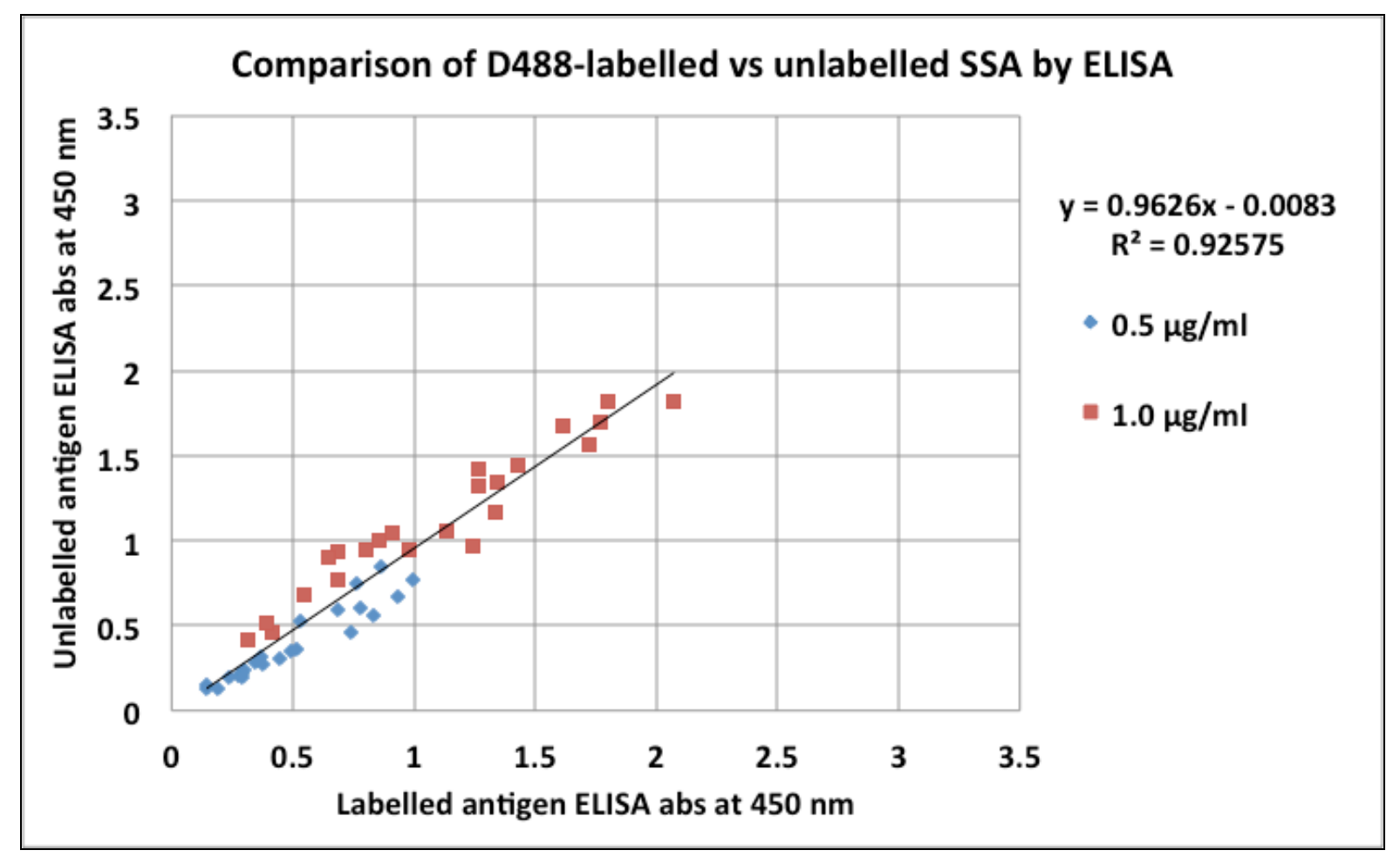

Figure 10 Comparison of labelled \& unlabelled SSA antigen by ELISA

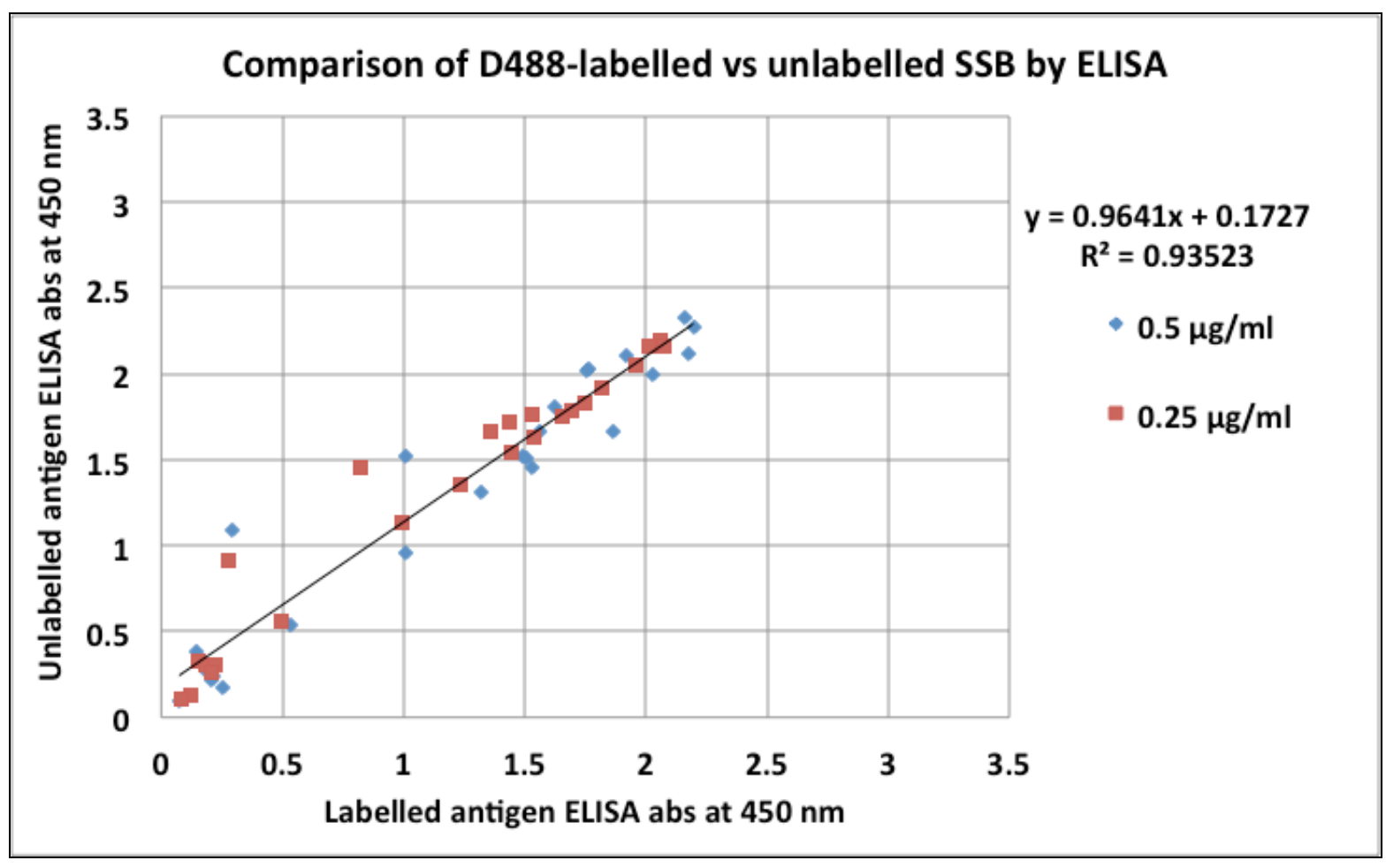

Figure 11 Comparison of labelled \& unlabelled SSB antigen by ELISA 


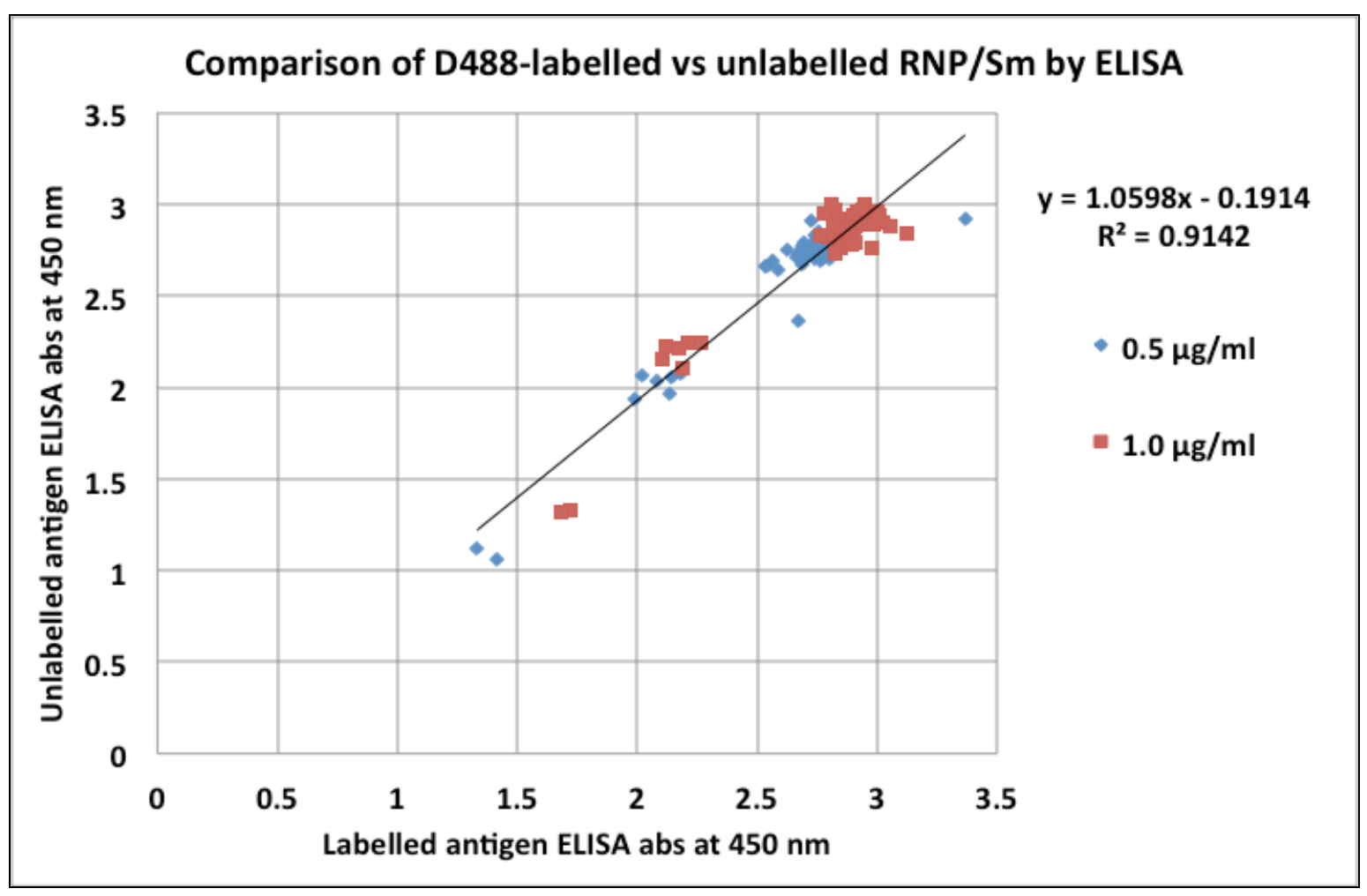

Figure 12 Comparison of labelled \& unlabelled RNP/Sm antigen by ELISA

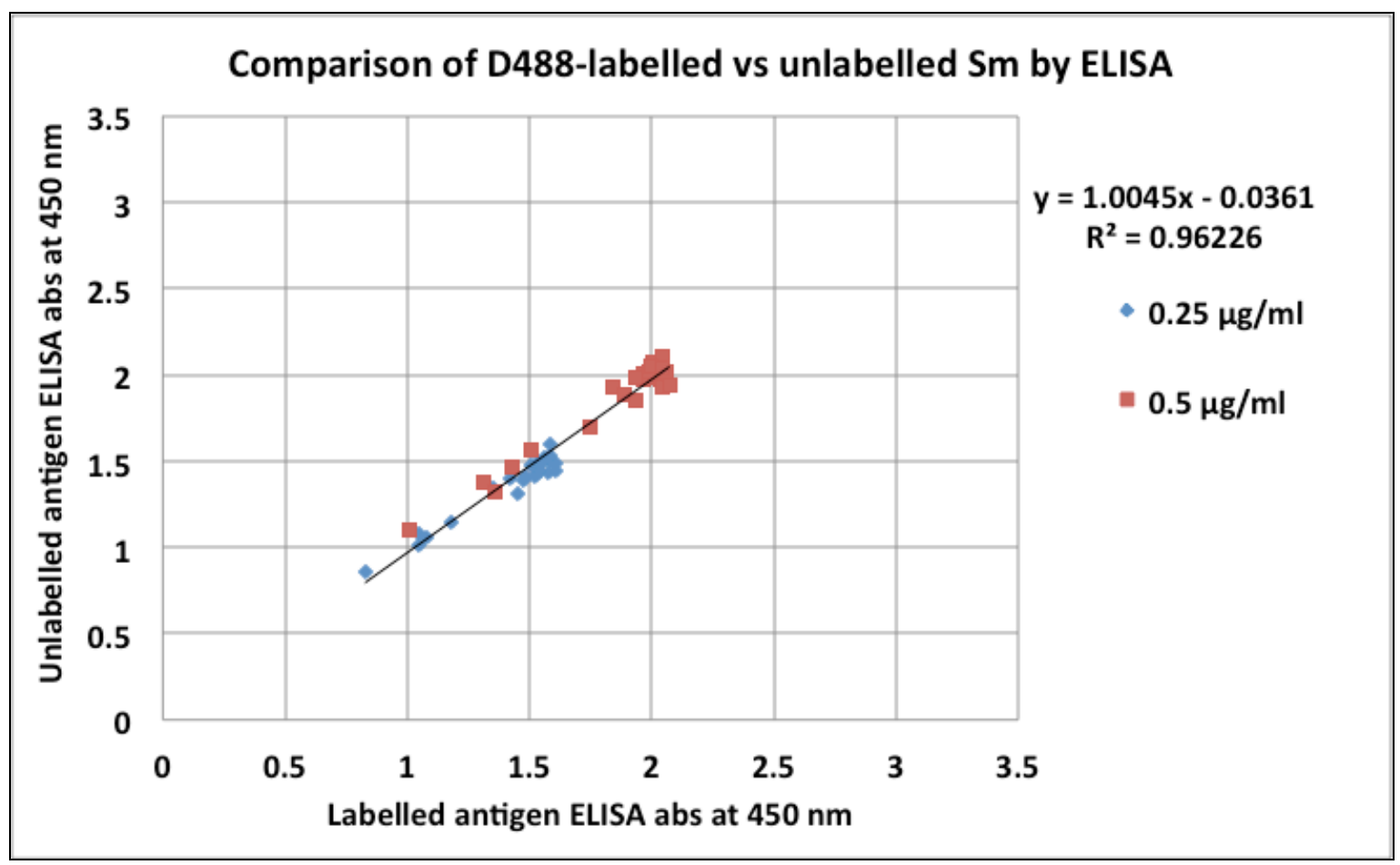

Figure 13 Comparison of labelled \& unlabelled Sm antigen by ELISA

Figures 10-13 Comparison of labelled and unlabelled antigens by ELISA Unlabelled and D488-labelled antigens (RNP/Sm, Sm, SSA, and SSB) labelled at a ratio of 5:1 analysed by ELISA at two different antigen coating concentrations. The ELISA data of unlabeled and labelled antigens was plotted against each other and linear regression analysis performed. The values of the $X \& Y$-axes are ELISA absorbances measured at $450 \mathrm{~nm}$. 


\subsection{Fluorescent Intensity of D488-Labelled Proteins}

To determine the both the detection limit and level of fluorescent intensity relative to the mass of protein loaded into a gel 2-fold serial dilutions were prepared for the antigens (SSA, and RNP/Sm) labelled with D488 at a 5:1 ratio. These were examined by SDS-PAGE. The gel was viewed using blue light excitation through an amber filter. The gels were also stained with Coomassie blue and the images captured. The fluorescent and Coomassie images were compared.

In figure 14 it can be seen that the relative fluorescent intensity increases relative to amount of protein loaded into the gel. This is true for both the antigens examined. Examining the fluorescent images (Fig 14) the limit of detection for SSA is $0.0613 \mu \mathrm{g}$ and the limit of detection for RNP/Sm is $0.125 \mu \mathrm{g}$. Examining the Coomassie images the limit of detection for SSA is $0.5 \mu \mathrm{g}$ and the limit of detection for RNP/Sm is 0.25 $\mu \mathrm{g}$. 


\section{Comparison of fluorescence and Coomassie stain for D488-labelled SSA and RNP/Sm antigens}

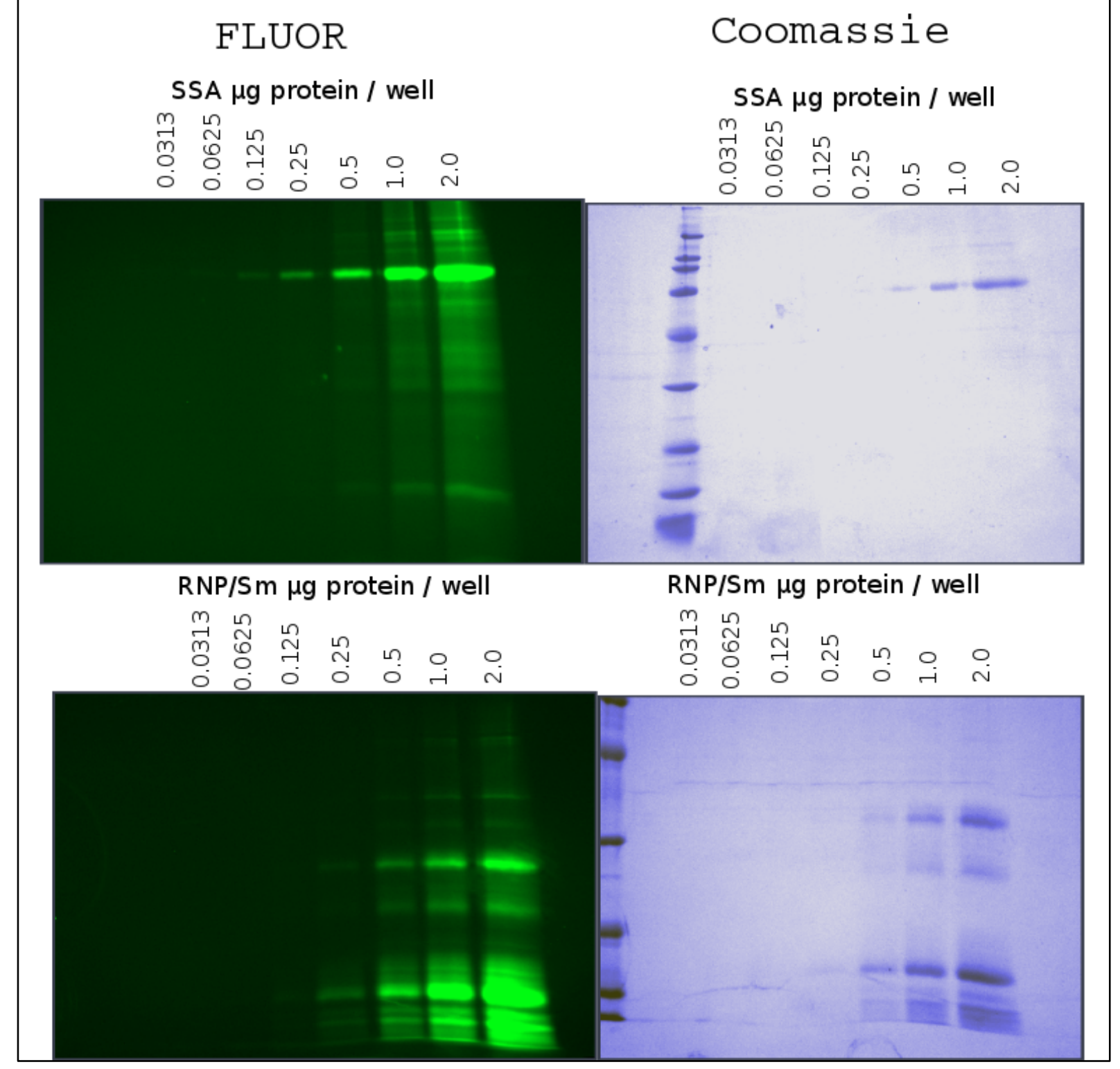

Figure 14 Fluorescence vs Coomassie for D488-labelled antigens 2-fold serial of the proteins SSA, SSB, RNP/Sm, and Sm labelled with D488 examined by SDS-PAGE. The gel was viewed through an amber filter using blue light excitation using an Invitrogen Safe Imager ${ }^{\mathrm{TM}}$ transilluminator, and the image captured using a Gel Doc ${ }^{\mathrm{TM}}$ XR (Bio-Rad). The gels were then fixed and stained with Coomassie blue and an image taken using white light transillumination in the Gel Doc ${ }^{\text {TM }}$ XR (Bio-Rad). Images were analysed using Quantity One 1-D (BioRad) software. 


\subsection{Comparison of D488 and AF488}

\subsubsection{Comparison of the relative fluorescent intensity of D488 and AF488 labelled ENAs}

To examine whether there was any difference between D488 and AF488 labelledantigens (SSA, SSB, RNP/Sm, Sm) and their respective fluorescent intensities the D488-labelled and AF488-labelled antigens were examined by SDS-PAGE. The gels were visualised with using blue light excitation and relative fluorescent intensity of the bands quantified (Fig. 15-B, D, F, \& H). The same gels were then fixed and stained with Coomassie blue and the relative signal intensity of the bands quantified (Fig. 15$A, C, E, \& G)$. Although the amount of protein loaded into each well was calculated to be $1 \mu \mathrm{g}$ it can be observed by visual examination and by examining the relative signal intensities of the Coomassie gels of all the antigens, that quantity of AF488labelled antigens present is greater than that of the D488-labelled antigens. Despite this the relative fluorescent intensity of the D488 labelled antigens produces a stronger signal than the AF488 labelled antigens. This indicates that the proteins labelled with the D488 dye produce a stronger fluorescent signal those labelled with AF488. Examining the bands in the SSB and Sm Coomassie images, it can be observed that there is batch-batch variation of the protein subunits, between D488 and AF488-labelled antigen samples. The quality control processes used to produce the antigen ensures that the antigen subunits are approximately the same between batches and that batch-batch variation is limited to the ratio of antigen subunits present in each sample. Thus batch-batch variation should not have an effect on the observations made regarding the differences in relative fluorescent intensity.

\subsubsection{Comparison of D488 and A488 by CIE}

To determine whether there was any difference between D488-labelled and AF488labelled antigens (SSA, SSB, RNP/Sm, and Sm) when used for CIE, three CIE assays were performed per antigen with different serum samples from the Wellington Hospital Immunology Department serum bank (Fig. 16). When a comparison was made between the precipitates of the SSA CIE assays the only observable difference was that the D488 precipitates were slightly brighter. When examining the images from the SSB CIE assays there was a difference in the precipitation pattern of the positive controls. The precipitate of the D488 positive control forms a wide spread arc and the precipitate of the AF488 positive control forms two distinct bands. However, 
there appeared to be no difference in the appearance of the negative control or the patient sample. There were no noticeable differences between D488 and AF488 in any of the RNP/Sm CIE assays. In contrast the Sm CIE had significant differences between D488 and AF488 results. The D488 precipitates were brighter and better resolved than the AF488 precipitates, and the AF488 negative control had a high background noise. Representative images are shown in Fig 16. 


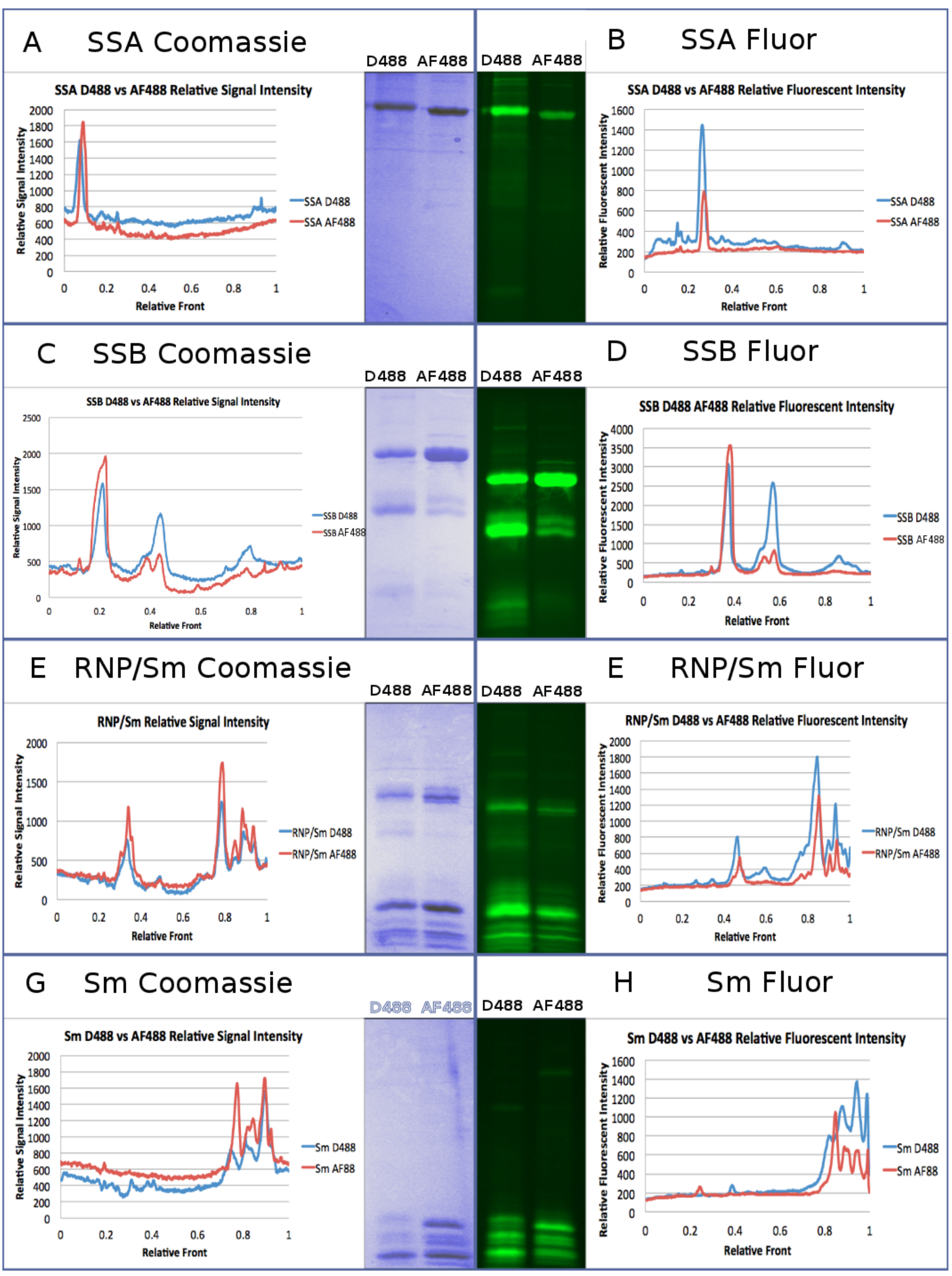

\section{Figure 15 Comparison of D488 and AF488 labelled antigens}

The D488-labelled and AF488-labelled antigens (SSA, SSB, RNP/Sm, and Sm) were examined by SDS-PAGE. The gels were visualised with using blue light excitation and relative fluorescent intensity of the bands quantitated (Fig. 15-B, D, $F, \& H)$. The gels were fixed and stained with coomasie blue and the relative signal intensity of the bands quantitated (Fig. 15-A, C, E, \& G). 
SSA Sample $1 \quad$ SSA Sample 2 SSA Sample 3

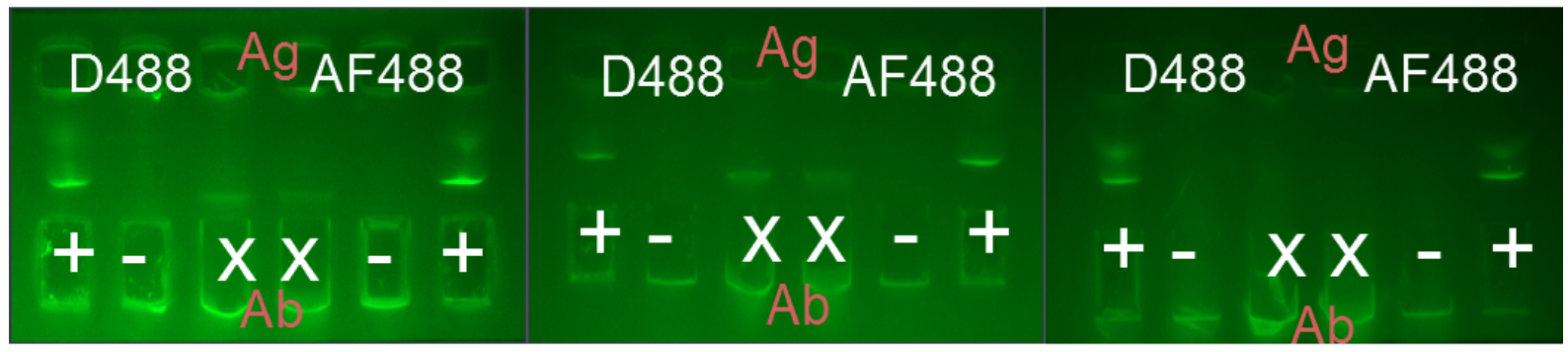

SSB Sample 1

SSB Sample 2

SSB Sample 3

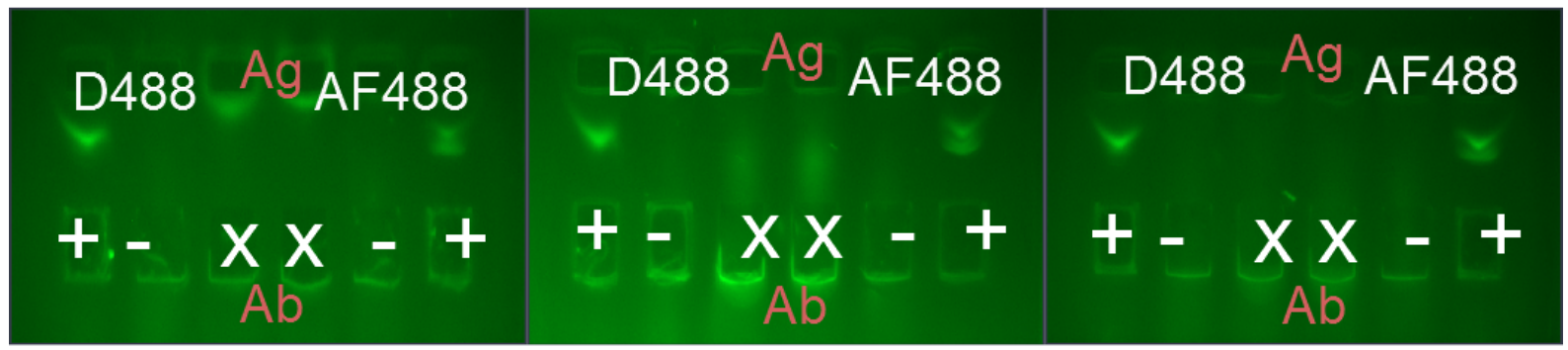

RNP/Sm Sample $1 \quad$ RNP/Sm Sample 2 RNP/Sm Sample 3

\begin{tabular}{|c|c|c|c|c|c|c|c|}
\hline D488 & $\mathrm{Ag}$ AF488 & $\mathrm{D} 48$ & $\mathrm{Ag} \mathrm{A}$ & F48 & & D4 & $\mathrm{Ag}$ AF488 \\
\hline 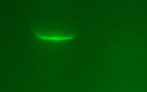 & -- & - & & & - & & - \\
\hline+ & $X_{A b} X-+$ & +- & $X X$ & & & +- & $x x=$ \\
\hline
\end{tabular}

Sm Sample 1

Sm Sample 2

Sm Sample 3

\begin{tabular}{|c|c|c|c|c|c|c|}
\hline D488 & $\mathrm{Ag}_{\mathrm{AF}} 488$ & D488 & $\mathrm{Ag}_{\mathrm{A}}$ & $=488$ & D488 & $\mathrm{Ag}_{\mathrm{AF} 488}$ \\
\hline $\mathbf{a}$ & & $\mathbf{a}$ & 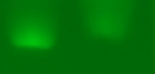 & & $a$ & 1a \\
\hline+ & $\underset{A b}{X}-+$ & $+=$ & $\frac{X X}{A B}$ & -+ & + & $x x=+$ \\
\hline
\end{tabular}

Figure 16 Comparison of D488 and AF488 by CIE

Three CIE assays were performed per ENA each with a different serum sample. Each $\mathrm{CIE}$ assay was loaded in duplicate with a set of serum samples; one positive sera control (+), one negative sera control (-), and a clinical sample from the Wellington Hospital Immunology department $(\mathbf{X})$. One serum set was run against D488-labelled antigen and the other against AF488-labelled antigen. CIE assays were performed with Apparatus 3 and the standard CIE method. In the Ag wells $1 \mu \mathrm{g}$ antigen is loaded into all wells. Using an amber filter under blue light excitation the precipitin reaction can be observed as bright horizontal bands. 


\subsection{Optimisation of Counter Immunoelectrophoresis}

\subsubsection{Agarose gel concentration and current}

To determine the effect of agarose concentration on the CIE assay, a series of replicate CIE assays were performed with increasing agarose concentrations. To examine the effect of electrical current applied during a CIE assay the agarose assay series was repeated twice again with two different applied currents. The results are shown in Fig. 17. As the agarose concentration increased the amount of background signal in the negative control increased (Fig. 17) without changing the resolution of the positive control. At the lowest agarose concentration $(0.4 \%)$ the gel would often deform when removed from the mold and be unusable for CIE. As the electrical current applied increased the length of time required for the assay to reach completion decreases (20 min decrease per $2 \mathrm{~mA}$ increase) and altering the current had no effect on the resolution of the positive control. However, at $7 \mathrm{~mA}$ the gels heat increased resulting in deformation at the terminal ends of the gel.

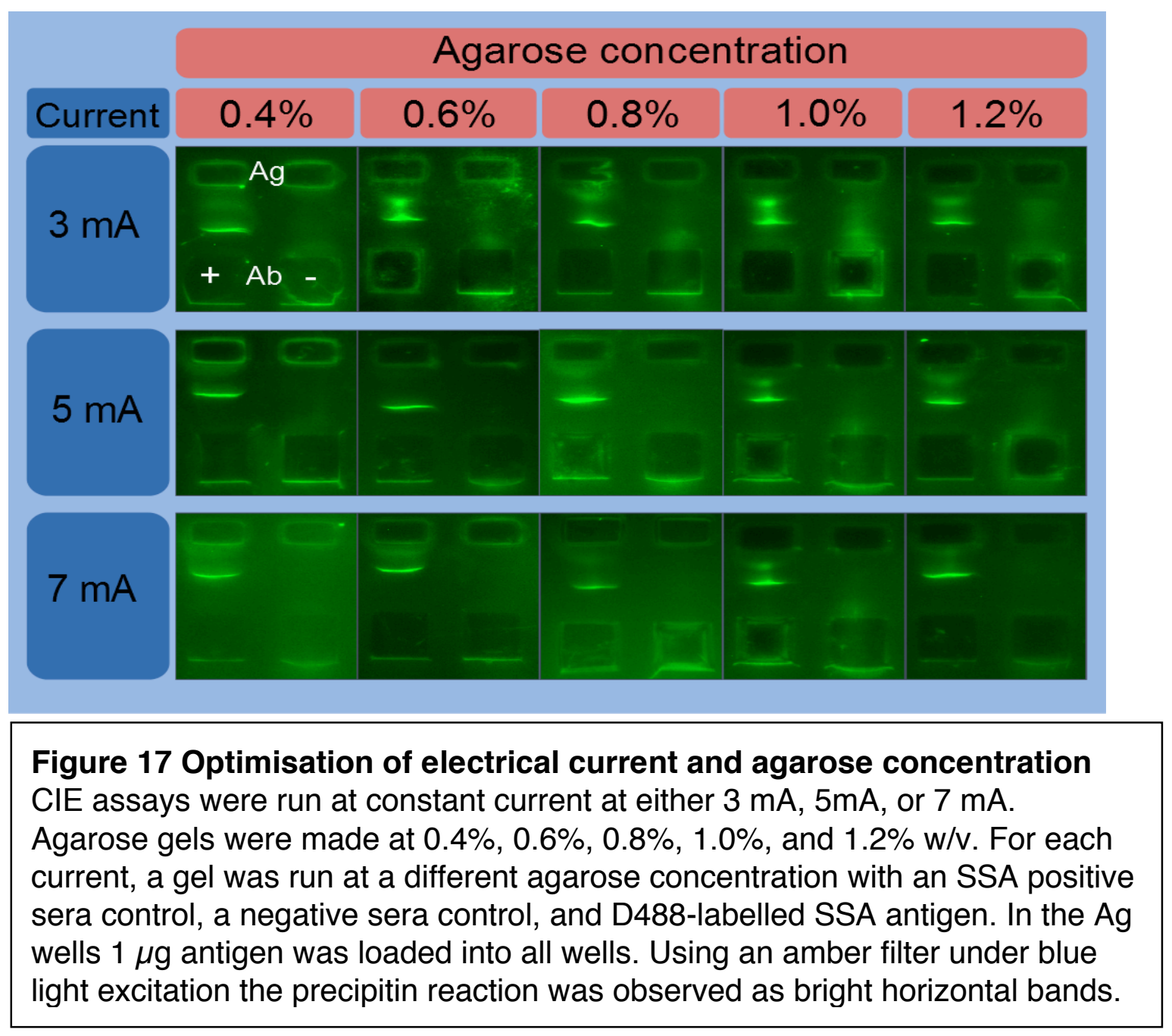




\subsubsection{Pre-electrophoresis time}

To examine the optimal period of time required for pre-electrophoresis two assays were performed using different pre-electrophoresis times. As the pre-electrophoresis time period increased, the further away from the antibody well the precipitation reaction occurred (Fig. 18A). Furthermore, as the pre-electrophoresis time period increased, there was a slight increase in the background present in the negative control (Fig. 18B). The length of pre-electrophoresis time period appeared to have no effect on the resolution of the positive control.

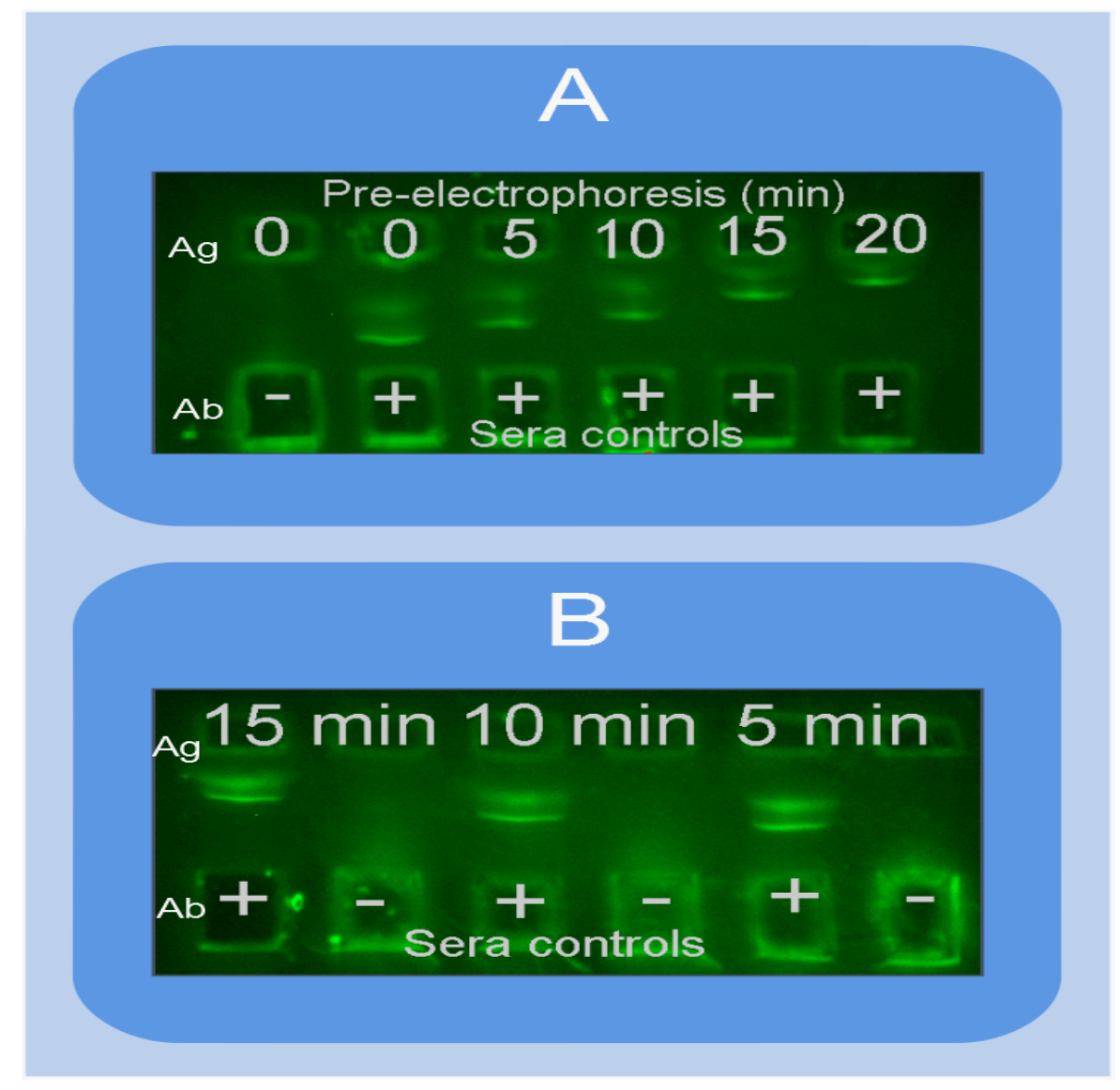

\section{Figure 18 Pre-electrophoresis}

Pre-electophoresis was staggered in 5 min intervals. Positive sera control (+), negative sera control (-). In the Ag wells $1 \mu \mathrm{g}$ antigen wass loaded into all wells. Using an amber filter under blue light excitation the precipitin reaction was observed as bright horizontal bands. 


\subsection{Optimisation of CIE Buffer}

\subsubsection{Calcium-lactate titration}

To examine the effect of Calcium-lactate on CIE assay resolution, a series of gels were made with buffers that had a different Calcium-lactate concentration. Using these gels a series of replicate CIE assays were performed (Fig. 19) with the SSA antigen and its respective positive and negative control sera. As the Calcium-lactate concentration increased so did the resolution of the lower titer sera samples. However, as the Calcium-lactate concentration increased so did the time taken for the assay to reach completion, with the $40 \mathrm{mM}$ calcium lactate assay unable to reach completion within $45 \mathrm{~min}$.

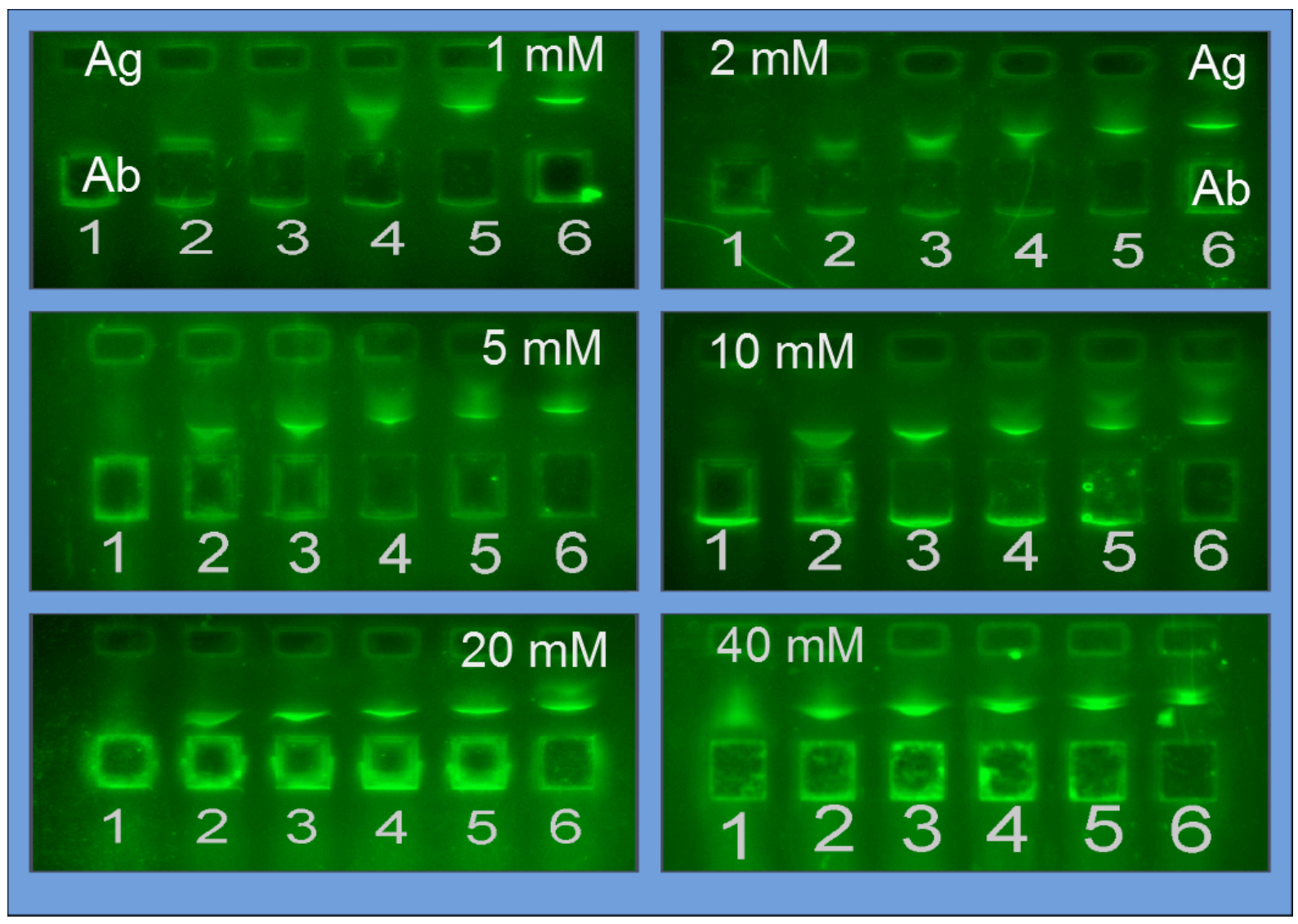

\section{Figure 19 CIE buffer calcium lactate titration}

A series of replicate $\mathrm{CIE}$ assays, each performed with a different gel made at different calcium lactate concentrations ( 1 to $40 \mathrm{mM}$ ). $0.1 \mu \mathrm{g}$ antigen was loaded into all Ag wells. A serum titration series was loaded into the Ab wells: 1 negative control; 2-5 2-fold sera dilution 1/16 to 1/2; 6 positive control. Using an amber filter under blue light excitation the precipitin reaction was observed as bright horizontal bands. 


\subsubsection{Optimisation of buffer pH}

To examine the effect of buffer $\mathrm{pH}$ on $\mathrm{CIE}$ assay resolution, three buffers were made (Buffers 1-3 see materials \& methods). Each buffer was made with the same constituents although with different concentrations of Tris and Tricine. This gave buffers 1-3 a different $\mathrm{pH}$ (calculated to be $\mathrm{pH} 8.4,8.6$, and 8.8 respectively) but the same relative conductivity $(0.19-0.2 \mu \mathrm{M} / \mathrm{s})$. Each buffer was heated between 15$40^{\circ} \mathrm{C}$ and its $\mathrm{pH}$ measured (Fig. 20). The change in $\mathrm{pH}$ as temperature increased was approximately linear. The difference in $\mathrm{pH}$ step-wise between the 3 buffers was approximately $0.2 \mathrm{pH}$ units and the rate of $\mathrm{pH}$ change for each of the buffers was approximately the same.

Three different CIE gels were made using each of the 3 buffers. These were used to run 3 replicate CIE assay using an SSB sera titration series (Fig. 21). When the 3 images from the 3 assays were compared there was no difference in band resolution, completion time, or difference in the negative control background.

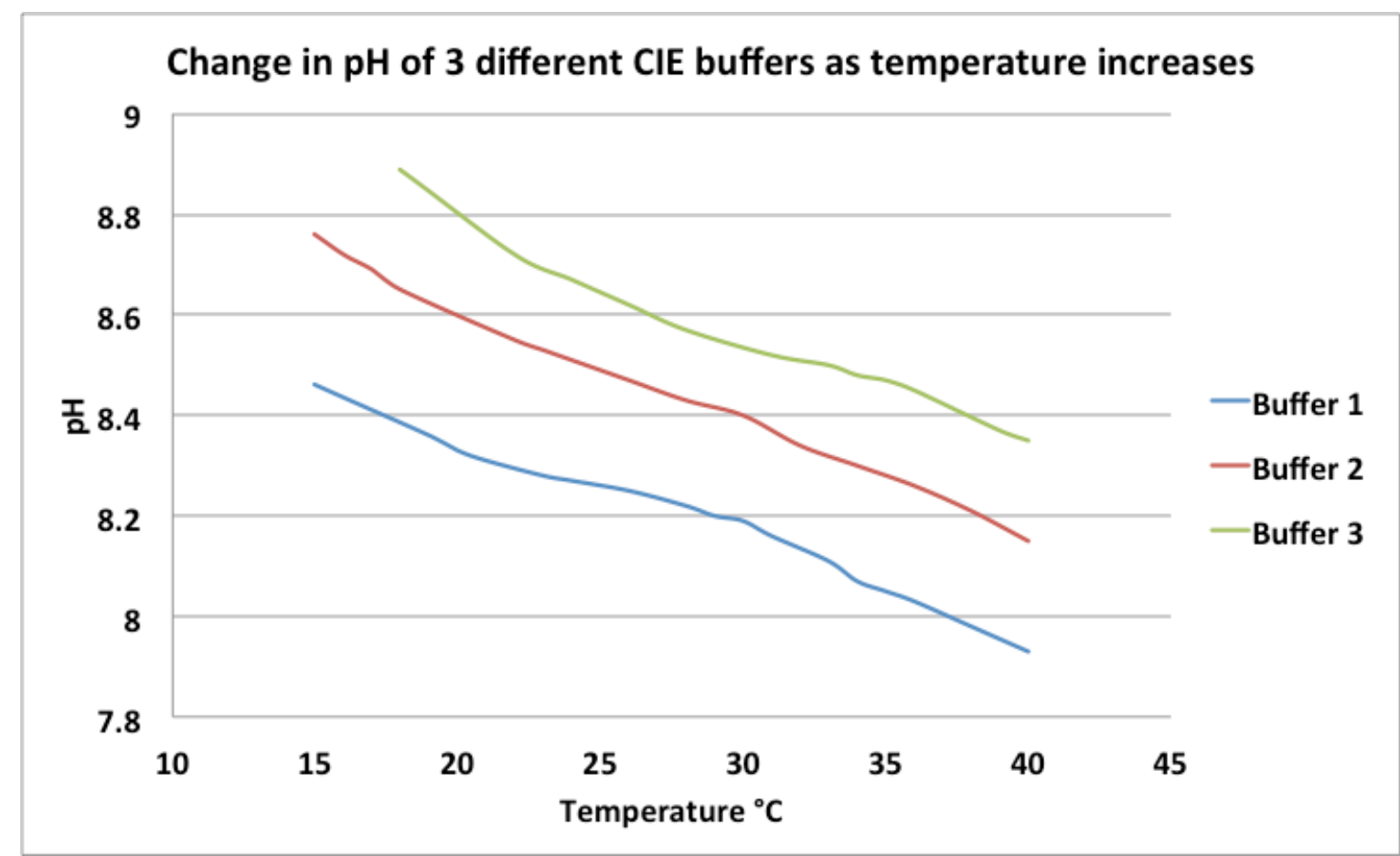

\section{Figure 20 Buffer $\mathrm{pH}$ vs Temperature}

Three buffers with identical constituents but at different concentrations were prepared. The $\mathrm{pH}$ of each buffer was measured while it was simultaneously being heated. The graph shows the change in $\mathrm{pH}$ as temperature increases for each of the three buffers. 


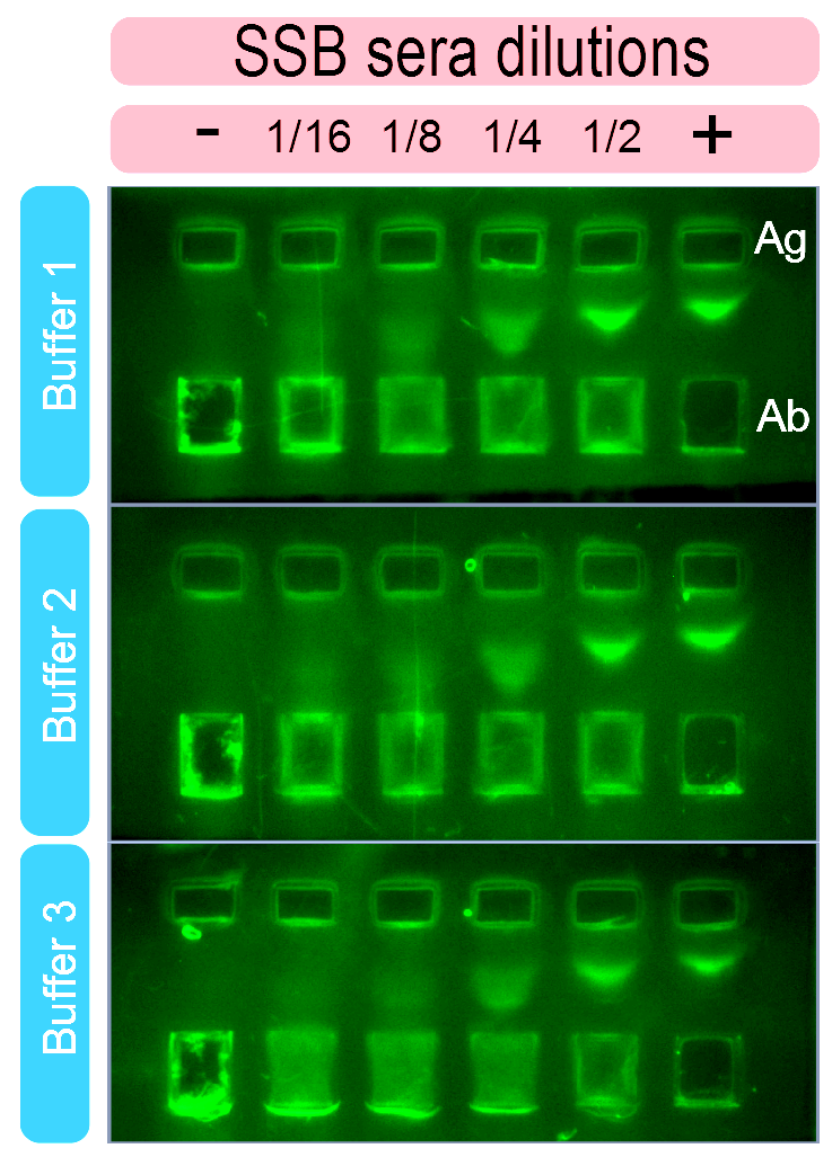

\section{Figure 21 The effect of buffer $\mathrm{pH}$ on CIE}

A series of replicate $\mathrm{CIE}$ assays, each performed with a different gel made with Buffers 1-3. In the Ag wells $0.1 \mu \mathrm{g} \mathrm{SSB}$ antigen was loaded into all wells. A serum titration series was loaded into the Ab wells: Lane1 negative control; Lanes 2-5 2-fold sera dilution 1/16 to 1/2;Lane 6 positive control. Using an amber filter under blue light excitation the precipitin reaction was observed as bright horizontal bands.

\subsubsection{Optimisation of buffer Tris-Tricine concentration}

To examine the effect of Tris-Tricine concentration, 3 buffers were made with increasing Tris-Tricine concentrations; Buffers $1 \mathrm{~A}$ (45 mM Tris and $10 \mathrm{mM}$ Tricine), 2A (90 mM Tris and $20 \mathrm{mM}$ Tricine), and $3 \mathrm{~A}$ (180 mM Tris and $40 \mathrm{mM}$ Tricine). Three different $\mathrm{CIE}$ gels were made using each of the 3 buffers. These were used to run replicate CIE assays using an SSA sera titration series (Fig. 22). As The Tris-Tricine concentration increased the resolution of the precipitin bands decreased. There was no change in the background of the negative control. Also gels made with Buffer $1 \mathrm{~A}$ would sometimes deform during electrophoresis. 


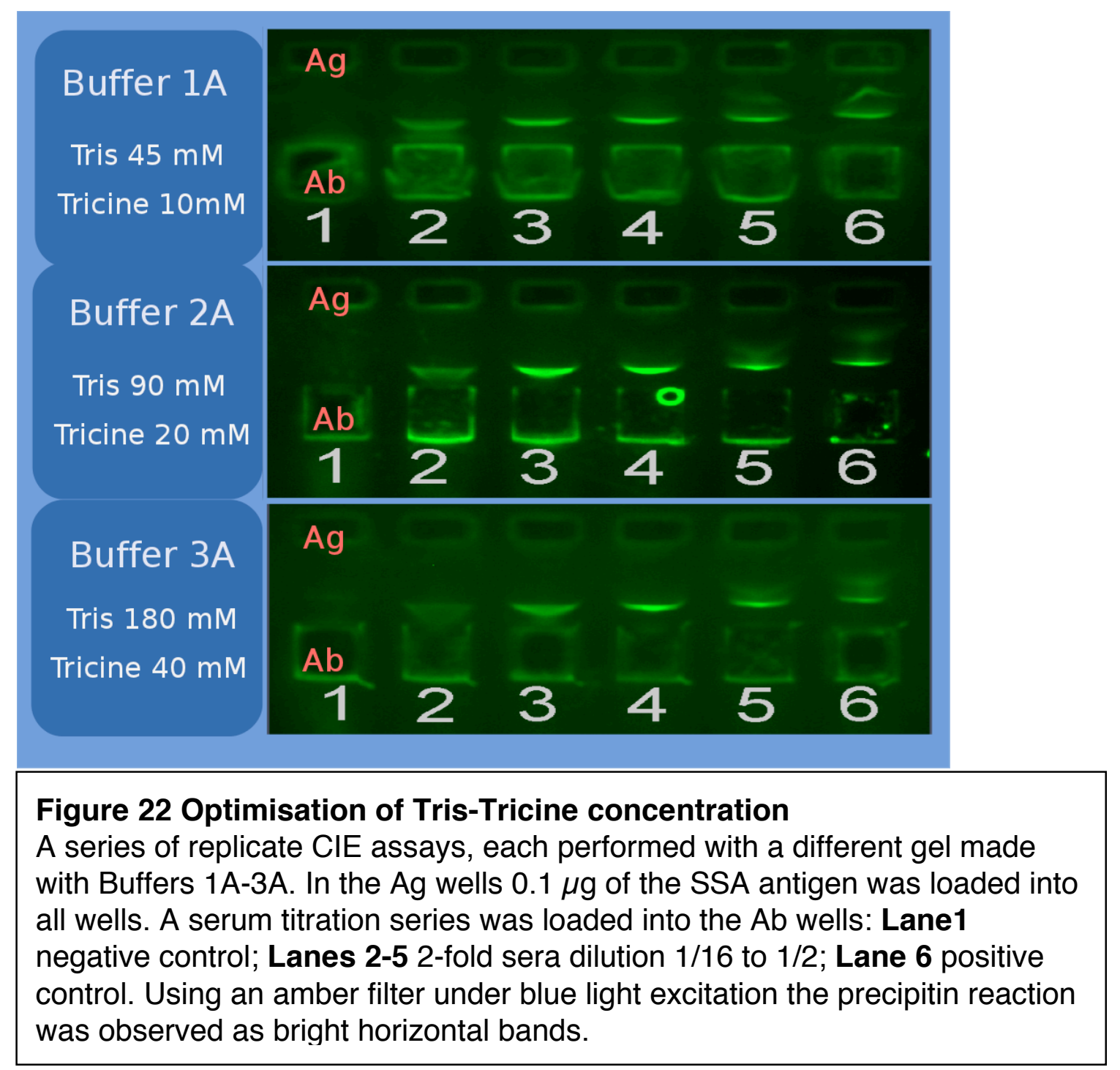

\subsection{Antigen concentration}

\subsubsection{Antigen titration}

To examine the optimal concentration of antigen required for CIE a 2-fold antigen titration series was created with range $80 \mathrm{ng} / \mu \mathrm{l}$ to $10 \mathrm{ng} / \mu \mathrm{l}$ for SSA, SSB, RNP/Sm, and $\mathrm{Sm}$. ClE assays were performed with each titration series and their corresponding positive and negative control sera (Fig. 23). The following SSA antigen concentrations are suitable for $\mathrm{CIE} 10,20$, and $40 \mathrm{ng} / \mu \mathrm{l}$ as the band fluorescence was clearly visible and bands were clearly defined. For SSB, RNP/Sm, and Sm the antigen concentrations suitable for CIE are 20, and $40 \mathrm{ng} / \mu \mathrm{l}$ (Fig. 23). 


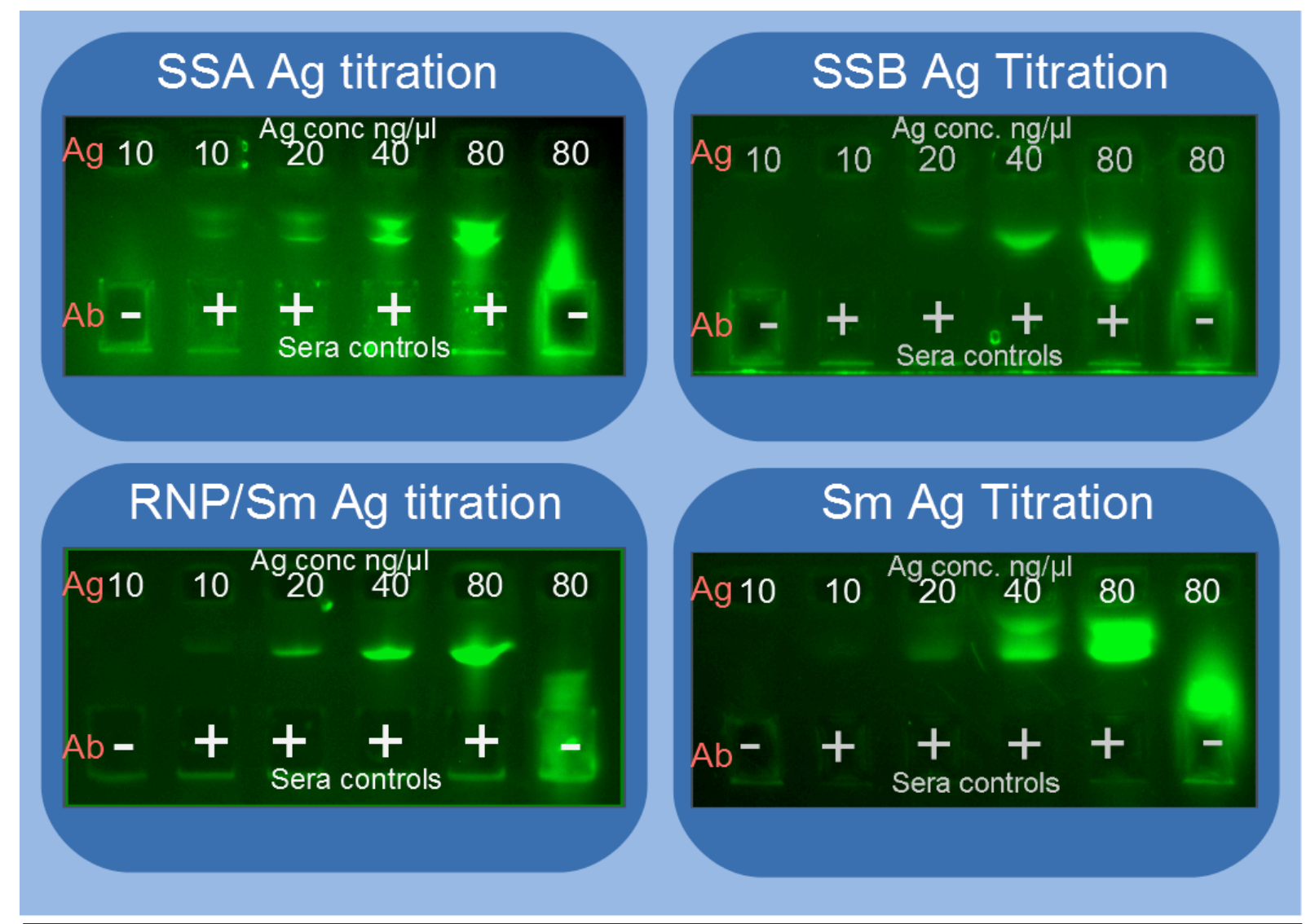

Figure 23 CIE antigen titration

A series of replicate $\mathrm{CIE}$ assays, each performed with a different a different antigen. An antigen titration series was loaded into the Ag wells: 10, 20, 40, \& 80 $\mathrm{ng} / \mathrm{\mu l}$. For each antigen a respective positive sera control (+), and negative sera control (-) was loaded into the Ab wells. Using an amber filter under blue light excitation the precipitin reaction was observed as bright horizontal bands.

\subsubsection{Antigen concentration vs sera titration series}

To examine the effect of antigen concentration on band resolution a 2-fold serum titration series was created (range $1 / 1$ to $1 / 256$ ) and this series was examined by $\mathrm{CIE}$ assay at each of the previously identified antigen concentrations (Figs 24-27).

As antigen concentration increased so did the fluorescence intensity and consequently visibility of the bands. Fluorescence intensity and visibility is strongest at an antigen concentration of $40 \mathrm{ng} / \mu \mathrm{l}$. At an SSA antigen concentration of $10 \mathrm{ng} / \mu \mathrm{l}$ the bands are noticeably less defined than at 20 , and $40 \mathrm{ng} / \mu \mathrm{l}$ (Fig. 24). Likewise, for all the antigens, there is no difference in the definition of bands between 20 , and 40 $\mathrm{ng} / \mu \mathrm{l}$. Notably, the presence of a secondary band correlated with an increasing sera titer. The limit of detection defined as a visible band clearly differentiable from the negative control for SSA, RNP/Sm, and Sm was at the 1/64 sera dilution. For SSB it was at the $1 / 4$ sera dilution. 
SSA sera titration at 3 different Ag concentrations

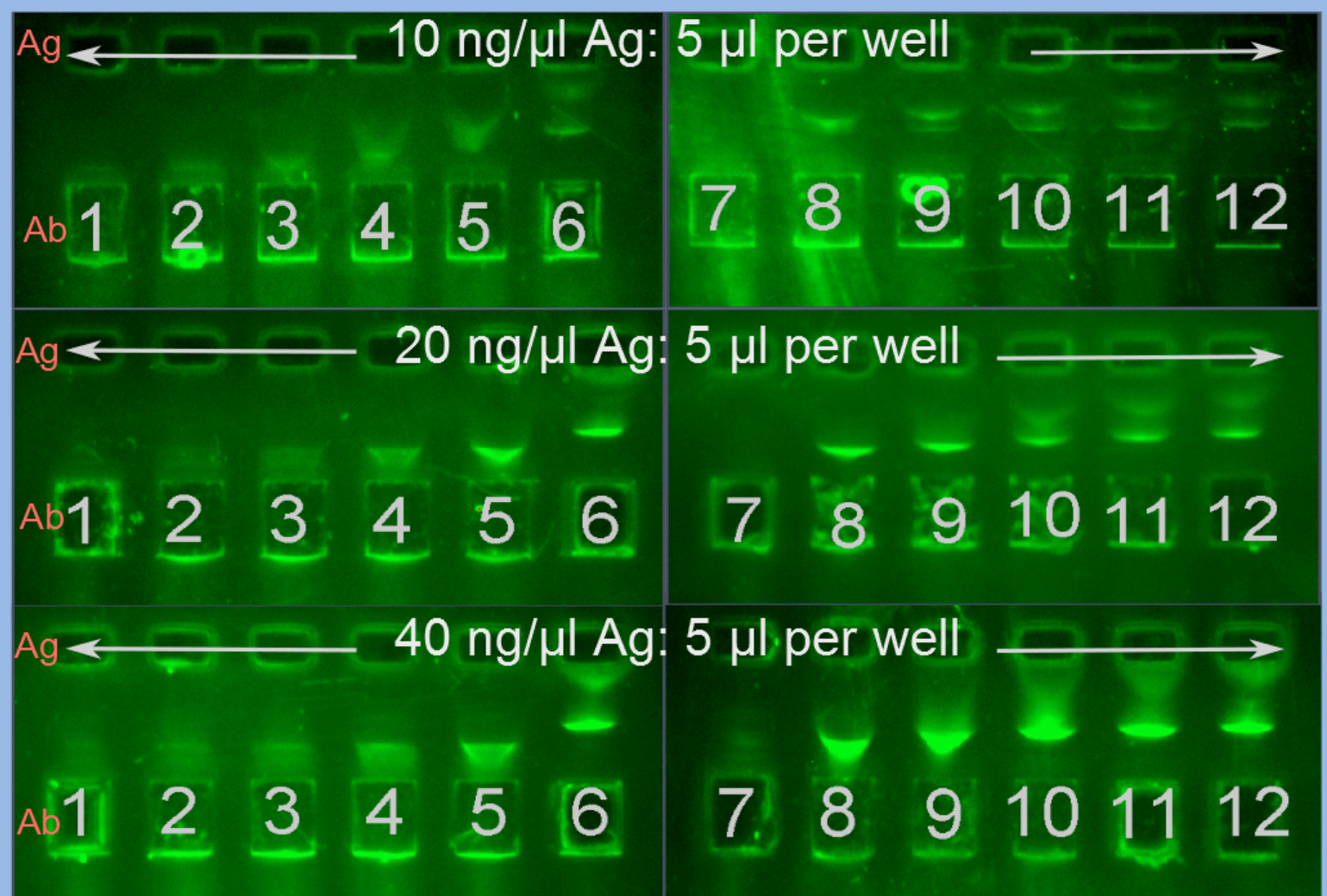

Figure 24 SSA sera titrations

SSB sera titration at 2 different Ag concentrations

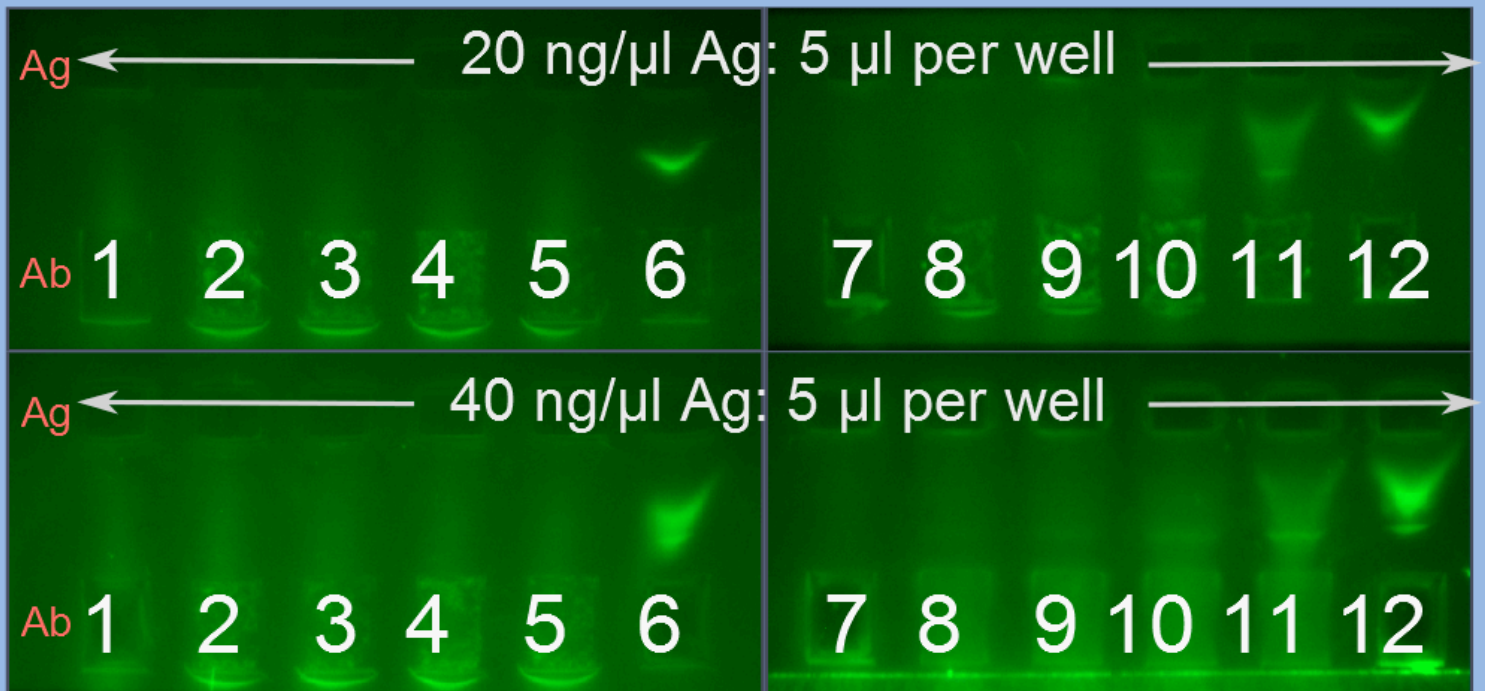

Figure 25 SSB sera titrations 


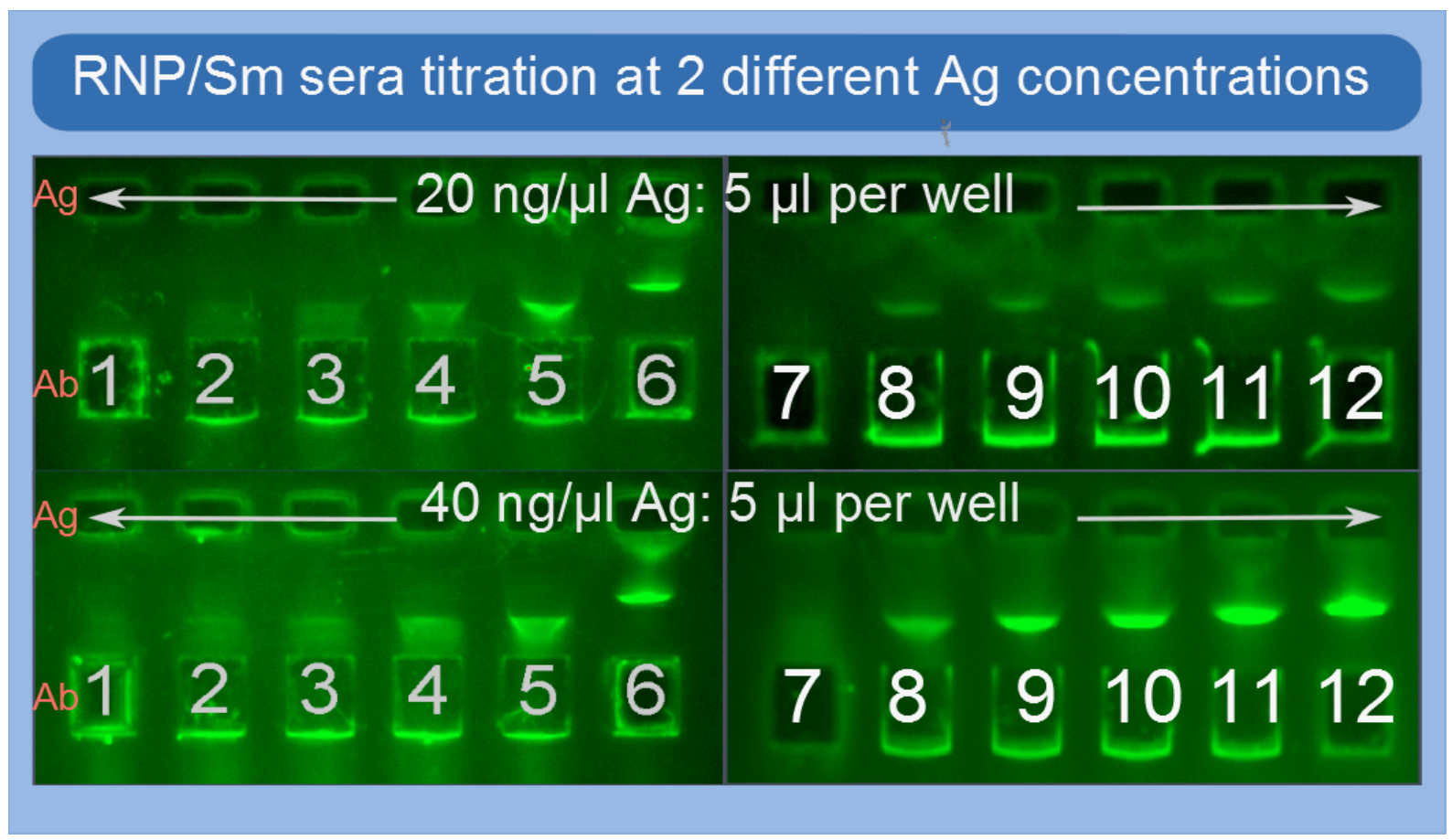

Figure 26 RNP/Sm sera titrations

Sm sera titration at 2 different $\mathrm{Ag}$ concentrations

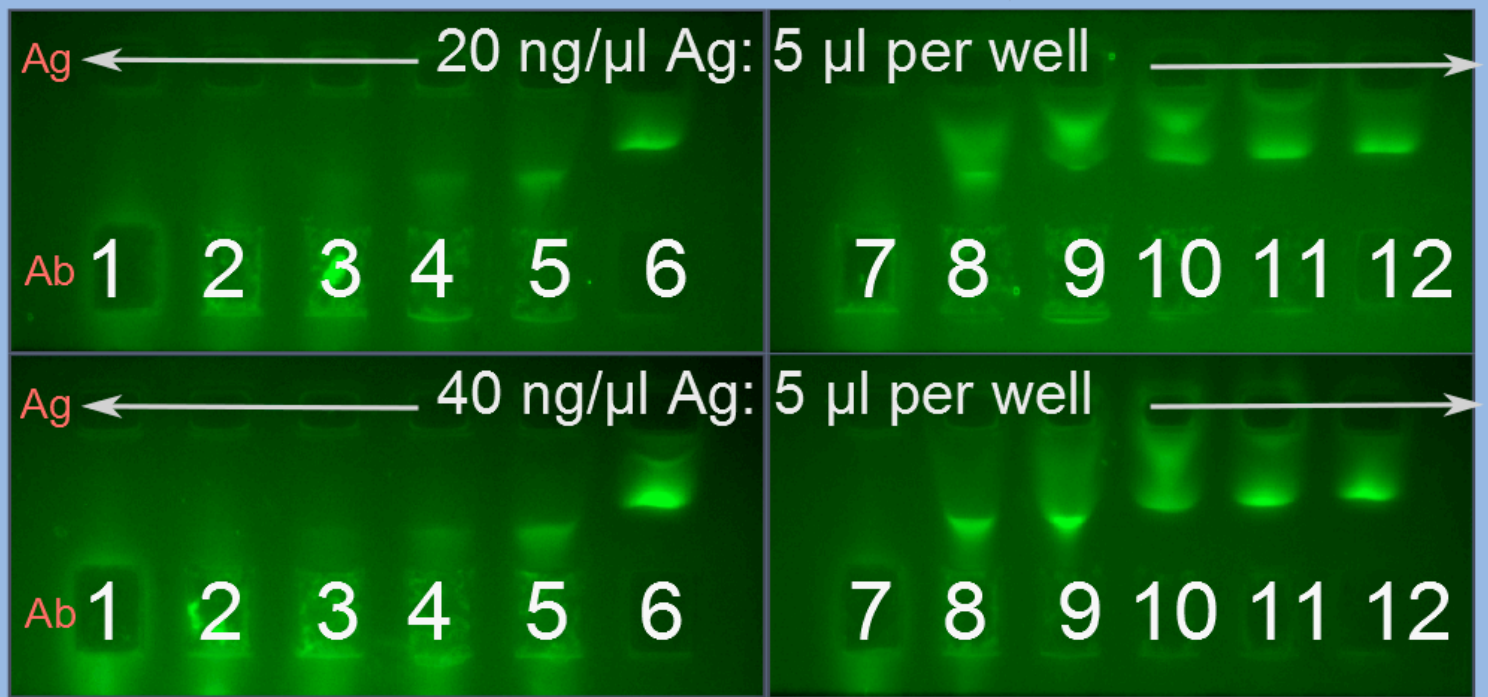

\section{Figure $27 \mathrm{Sm}$ sera titrations}

\section{Figures 24-27}

Replicate CIE assays, each performed with a different antigen at different antigen concentrations: $10,20, \& 40 \mathrm{ng} / \mu \mathrm{l}$ for SSA and 20, \& 40ng/ $\mu$ l for SSB, RNP/Sm and Sm. In the Ag wells $5 \mu$ l antigen was loaded into each well. A serum titration series was loaded into the Ab wells; Lane1 negative control, Lanes 2-5 2-fold sera dilutions 1/256 to 1/32, Lane 6 positive control, Lane 7 negative control, Lane 8-11 sera dilutions $1 / 16$ to $1 / 2$, Lane 12 positive control. Using an amber filter under blue light excitation the precipitin reaction was observed as bright horizontal bands. 


\subsection{Antigen Analytical Specificity}

To examine the analytical specificity of the antigens SSA, SSB, RNP/Sm, and Sm four CIE assays were performed with a single antigen and 6 different ENA reactive sera; anti-SSA, anti-SSA/SSB, anti-RNP, anti-Sm, anti-Jo-1, and anti-Scl-70 (Fig. 28). As expected, the SSA antigen reacted with the anti-SSA sera, and anti-SSA/SSB sera; the SSB antigen reacted with anti-SSB sera; the RNP/Sm antigen reacted with the anti-RNP, and anti-Sm sera; the Sm antigen reacted with the anti-Sm sera. There was no non-specific cross reactivity of the antigens detected.

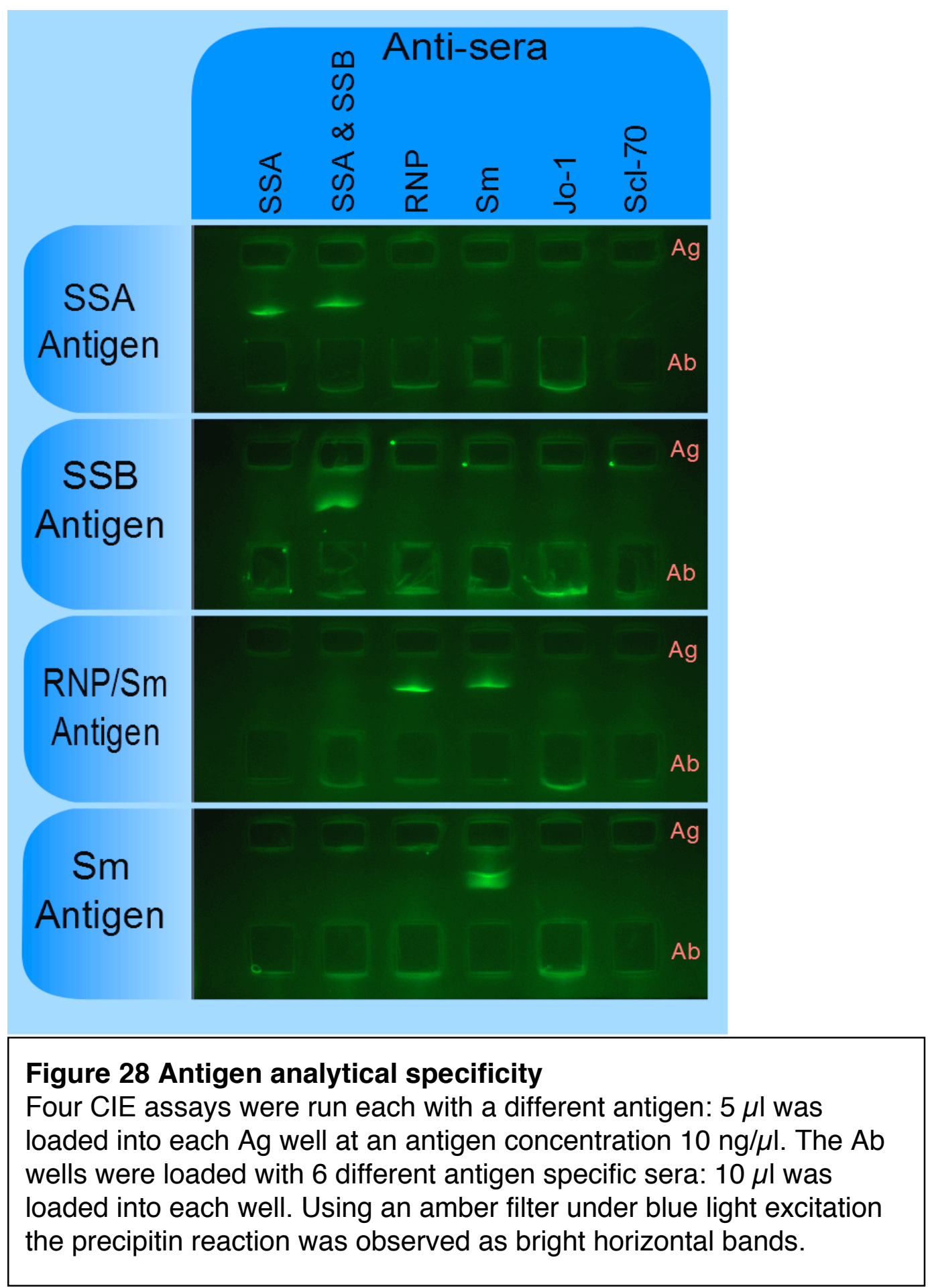




\subsection{Antigen Time Series}

$\mathrm{CIE}$ assays using the antigens SSA, SSB, RNP/Sm, and Sm were run against their respective positive sera control, a negative sera control, and a buffer blank. Images were taken periodically throughout the length of the assay (Figs 29 and 30). The presence of a defined precipitation band becomes observable after $12 \mathrm{~min}$ for all antigens. The end point of the assay is when the antigen runs through the gel past the negative control sera well. For the SSA and SSB antigens it took 30 min to reach the assay endpoint and for RNP/Sm and Sm it took 50 min to reach the assay endpoint. At the endpoint of the RNP/Sm assay there was low background fluorescence in the negative sera control lane that persisted 


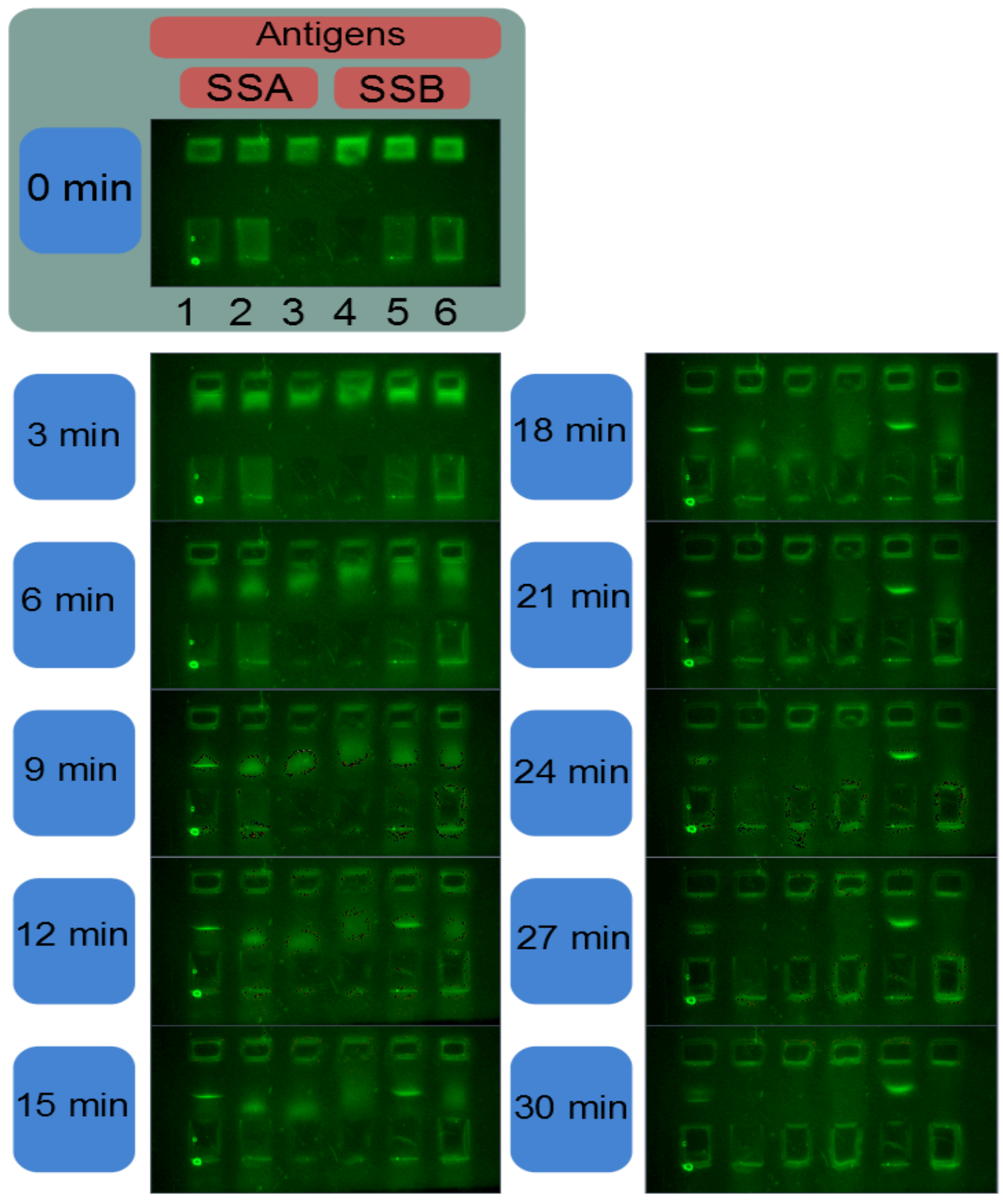

\section{Figure 29 SSA and SSB CIE time series}

A CIE assay was performed with two antigens (Lanes 1-3 SSA and Lanes 4-6 SSB): $5 \mu \mathrm{l}$ was loaded into each Ag well at an antigen concentration 10 $\mathrm{ng} / \mu \mathrm{l}$. The Ab wells were loaded with $10 \mu \mathrm{l}$ : Lanes 1 \& 5 positive control sera, Lanes 2 \& 4 negative control sera, Lanes 3 \& 6 buffer blank. Using an amber filter under blue light excitation the precipitin reaction was observed as bright horizontal bands. 


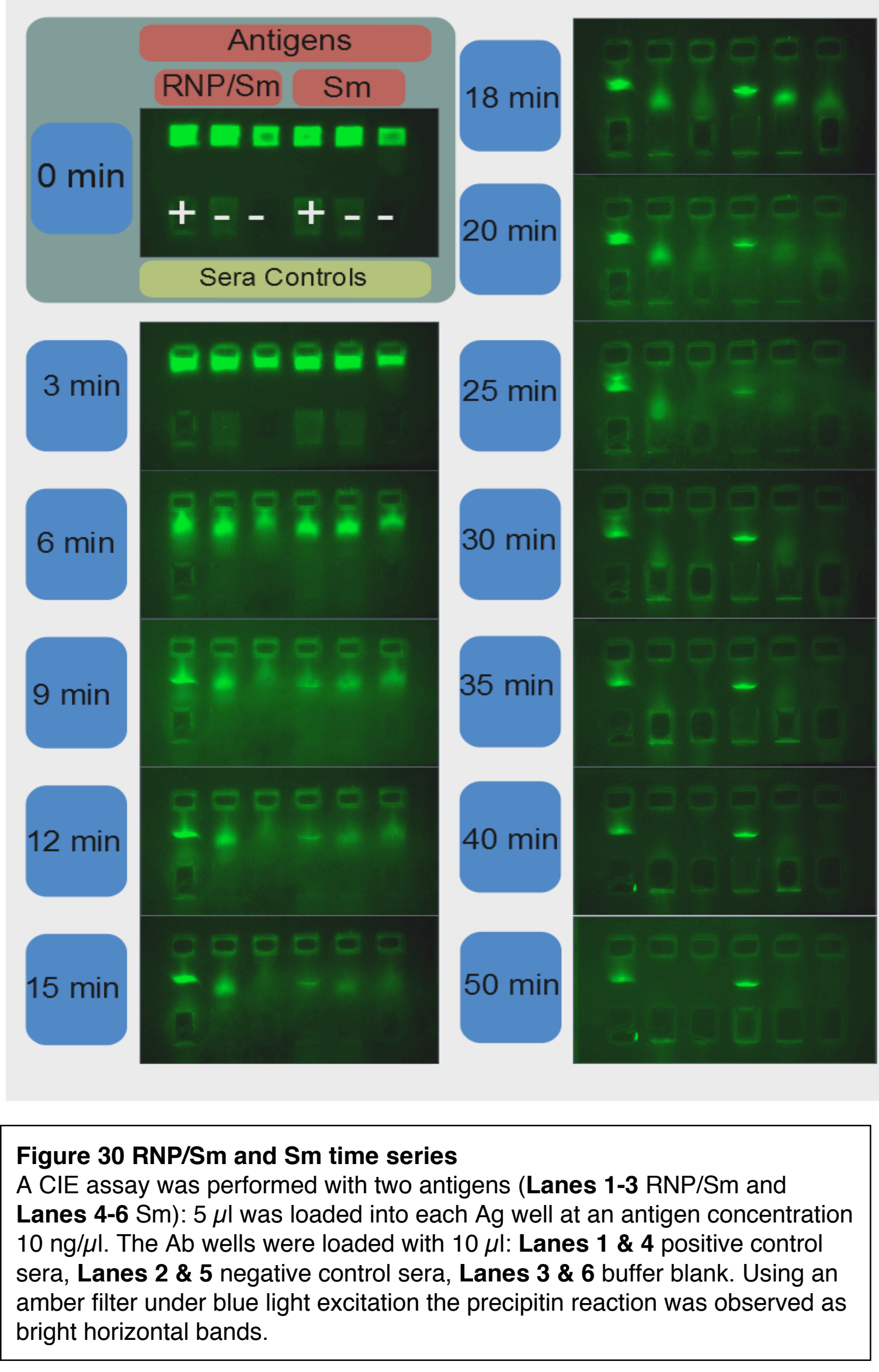




\subsection{Clinical Validation of the CIE Assays}

To validate the CIE assays for the SSA, SSB, RNP/Sm, and Sm antigens CIE assays were performed using 281 clinical samples from the Wellington Hospital Immunology Department serum bank. The presence of a clearly defined precipitin band at the end point of the assay was scored as a positive CIE result. Results that did not produce a clearly defined precipitin band were scored as a negative CIE result.

The 281 clinical samples were also examined by ELISA for each of the antigens SSA, SSB, RNP/Sm, and Sm. The detection cutoff for each specific antigen by ELISA was determined, by determining the $99 \%$ confidence interval of the mean ELISA score of a sample of negative controls (Frey et al., 1998). All true negative results are expected to lie within this interval. The upper confidence value of the mean is taken to be the ELISA cut off point. The ELISA endpoints for each respective antigen are summarised in Table 5. An ELISA score above the endpoint is considered positive and a ELISA score equal or below the endpoint is considered negative.

\begin{tabular}{ll}
\hline Antigen & ELISA Cutoff \\
\hline SSA & Negative $\leq 0.462<$ Positive \\
SSB & Negative $\leq 0.369<$ Positive \\
RNP $/$ Sm & Negative $\leq 0.266<$ Positive \\
Sm & Negative $\leq 0.280<$ Positive \\
& \\
\hline \hline Table 5. Endpoint cutoffs for anti-ENA ELISAS
\end{tabular}

Table 5. Endpoint cutoffs for anti-ENA ELISAs

\subsubsection{Correlation between CIE and ELISA clinical samples scores}

Data was collated into $2 \times 2$ tables and Cohen's kappa analysis was performed to compare the inter-rata agreement between the CIE and ELISA clinical sample results (Tables 6-9). To visualise the agreement between CIE and ELISA clinical sample positive results Venn diagrams were created (Fig. 31). The Cohen's kappa ( $k$ ) value for the inter-rata agreement between the SSA CIE and ELISA results was 0.89. This indicates a strong agreement between the observations of each assay. The $k$-values for the inter-rata agreement between the SSB, RNP/Sm, and Sm CIE and ELISA results were $0.48,0.7$, and 0.38 respectively and indicate a moderate, a substantial, and fair agreement between the observations, of each respective set of results. 


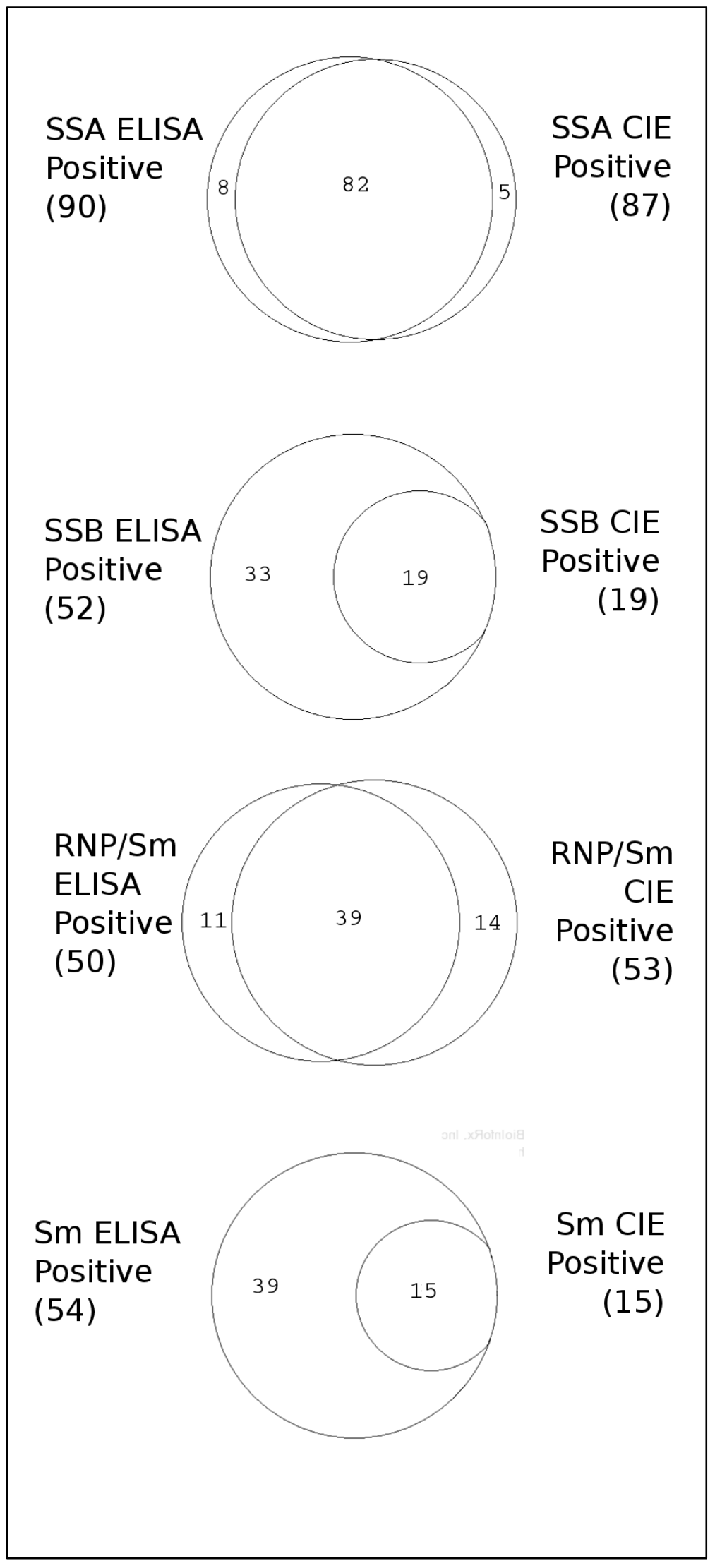

Figure 31 Overlap between CIE and ELISA positive results Area-proportional Venn diagrams were generated the BIOINFOX Venn diagram program 


\begin{tabular}{lccc}
\hline SSA & ELISA Positive & ELISA Negative & Total \\
\hline CIE Positive & 82 & 5 & 87 \\
CIE Negative & 8 & 186 & 194 \\
Total & 90 & 191 & 281 \\
& & & \\
\hline Cohen's kappa & 0.89 & & \\
\hline \hline
\end{tabular}

Table 6. Correlation between SSA CIE and ELISA clinical samples scores

\begin{tabular}{lccc}
\hline SSB & ELISA Positive & ELISA Negative & Total \\
\hline CIE Positive & 19 & 0 & 19 \\
CIE Negative & 33 & 229 & 262 \\
Total & 52 & 229 & 281 \\
& & & \\
Cohen's kappa & 0.48 & &
\end{tabular}

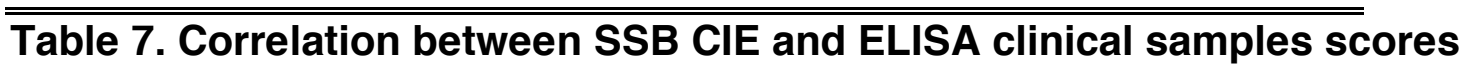

\begin{tabular}{lccc}
\hline RNP/Sm & ELISA Positive & ELISA Negative & Total \\
\hline CIE Positive & 39 & 14 & 53 \\
CIE Negative & 11 & 217 & 228 \\
Total & 50 & 231 & 281 \\
& & & \\
Cohen's kappa & 0.70 & &
\end{tabular}

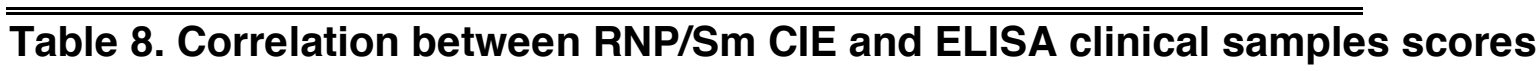

\begin{tabular}{lccc}
\hline Sm & ELISA Positive & ELISA Negative & Total \\
\hline CIE Positive & 15 & 0 & 15 \\
CIE Negative & 39 & 227 & 266 \\
Total & 54 & 227 & 281 \\
& & & \\
\hline Cohen's kappa & 0.38 & &
\end{tabular}

Table 9. Correlation between Sm CIE and ELISA clinical samples scores

\subsubsection{Correlation between clinical data and ELISA and CIE diagnostic assays}

Categorical clinical data was available for 156 of the 281 clinical sera samples tested. For the serum samples with categorical data available their corresponding CIE assay and ELISA results were grouped together into case groups. Case group 1 (categorical groupings consistent with SS) consisted of samples categorised 1-4; Case group 2 (categorical grouping consistent with other CTD) consisted of samples categorised 5 and 6; Case group 3 (control group) consisted of samples in 
category 7. The results of the clinical sera sample CIE assays and ELISAs in Case groups 1 and 2 were cross reference with the results in Case group 3 to determine the diagnostic specificity and sensitivity, PLR and NLR of the CIE assays and ELISAs (Tables 10 and 11).

The performance characteristics of the ENA CIE assay and ELISA results assigned to Case group 1 were examined (Table 10). The diagnostic specificity of all CIE assays and ELISAs were greater than or equal to $85.9 \%$. Furthermore, there was much more variation in the diagnostic sensitivity of the assays ranging between $12.5 \%-70.8 \%$. The specificity and the PLR of the SSA CIE assay were superior to that of the SSA ELISA. However, the sensitivity and NLR were approximately the same. The diagnostic specificity of the SSB CIE assay (98.9\%) was superior to that of the ELISA (89.1\%). The PLR of the SSB CIE assay was very high (15.33) compared not only with the SSB ELISA but with all other ENA CIE assays and ELISAs. The values for the SSB CIE diagnostic sensitivity and NLR were not as favorable (16.7\% and 0.84 respectively) compared to the diagnostic sensitivity and NLR of the SSB ELISA (54.2\% and $0.51 \%$ ) respectively. The values for PLR, diagnostic sensitivity and specific were lower for the RNP/Sm CIE (3.51, 45.8\%, and $87.0 \%$ respectively) than the RNP/Sm ELISA (5.11, 50\%, and 90.2\% respectively). Also the NLR value was higher for the RNP/Sm CIE (0.62) than the RNP/Sm ELISA (0.55). The values for diagnostic specificity, PLR, and NLR were higher for the Sm CIE assay (97.8\%, 5.75, and 0.89 respectively) than the Sm ELISA assay (85.9\%, 2.36, and 0.78 respectively). Notably, the PLR of the Sm CIE assay is approximately double that of the Sm ELISA. The diagnostic sensitivity of the Sm CIE assay (12.5\%) is approximately a third that of the Sm ELISA (33.5\%).

The performance characteristics of the ENA CIE assay and ELISA results assigned to Case group 2, were examined (Table 11). The diagnostic specificities for all ENA CIE assays and ELISAs in this Case group 2 were the same as those in Case group 1 analysis (Fig 10.) because the Control group (Case group 3) was the same for both. The diagnostic sensitivity of all assays was low ranging from (2.5\%-22.5\%). The values for PLR (range 0.92-3.45) and NLR (range 0.86-1.01) for all assays were poor. 


\begin{tabular}{|c|c|c|c|c|}
\hline & Sensitivity & Specificity & PLR & NLR \\
\hline SSA CIE & $70.8 \%$ & $90.2 \%$ & 7.24 & 0.32 \\
\hline SSA ELISA & $70.8 \%$ & $88.0 \%$ & 5.92 & 0.33 \\
\hline SSB CIE & $16.7 \%$ & $98.9 \%$ & 15.33 & 0.84 \\
\hline SSB ELISA & $54.2 \%$ & $89.1 \%$ & 4.98 & 0.51 \\
\hline RNP/Sm CIE & $45.8 \%$ & $87.0 \%$ & 3.51 & 0.62 \\
\hline RNP/Sm ELISA & $50.0 \%$ & $90.2 \%$ & 5.11 & 0.55 \\
\hline Sm CIE & $12.5 \%$ & $97.8 \%$ & 5.75 & 0.89 \\
\hline Sm ELISA & $33.3 \%$ & $85.9 \%$ & 2.36 & 0.78 \\
\hline
\end{tabular}

\begin{tabular}{|c|c|c|c|c|}
\hline & Sensitivity & Specificity & PLR & NLR \\
\hline SSA CIE & $22.5 \%$ & $90.2 \%$ & 2.30 & 0.86 \\
\hline SSA ELISA & $22.5 \%$ & $88.0 \%$ & 1.88 & 0.88 \\
\hline SSB CIE & $2.5 \%$ & $98.9 \%$ & 2.30 & 0.99 \\
\hline SSB ELISA & $10.0 \%$ & $89.1 \%$ & 0.92 & 1.01 \\
\hline RNP/Sm CIE & $22.5 \%$ & $87.0 \%$ & 1.73 & 0.89 \\
\hline RNP/Sm ELISA & $17.5 \%$ & $90.2 \%$ & 1.79 & 0.91 \\
\hline Sm CIE & $7.5 \%$ & $97.8 \%$ & 3.45 & 0.95 \\
\hline Sm ELISA & $20.0 \%$ & $85.9 \%$ & 1.42 & 0.93 \\
\hline
\end{tabular}

\section{Tables $10 \& 11$}

Using Case group 1 (Table 10) and Case group 2 (Table 11) clinical data the performance characteristics diagnostic sensitivity and specificity, positive likelihood ratio (PLR), and negative likelihood ratio (NLR) were determined for the each of the antigens (SSA, SSB, RNP/Sm, \& Sm) with their respective CIE assay and ELISAs.

\subsubsection{Western blot}

After testing the clinical sera samples by CIE assay and ELISA was completed, when the results of the CIE assay and ELISA differed, a Western blotting was attempted as a differential test. The blots were scored 0 for a negative result as compared to the negative control, 2 for a positive result as compared to the positive control, or 1 for an inclusive result as compared to the positive and negative controls. During testing it was observed that a high proportion of the Western blots were producing 
inconclusive results, with approximately half the samples tested scored as being inconclusive (See appendices for results). Therefore, further testing was stopped since the Western blot results were considered to be unusable for analysis because of the high proportion of inconclusive results, and the Western blotting method used was deemed to be insufficient as differential test.

\subsection{Double Immunodiffusion}

To determine the whether the D488-labelled RNP/Sm antigen would be suitable for DID a series of RNP/Sm double DID assays were performed. The serum samples used to examine the RNP/Sm DID assay were a selection of Wellington Hospital Immunology Department sera samples and selection of Arotec internal standards (Figs 32 \& 33). Clinical samples that were positive by ELISA and CIE, produced a precipitin band. None of the samples that were negative by ELISA and CIE produced a precipitin band. Also all the Arotec internal standards that were tested produced results that were consistent with known specificity of the sera tested.

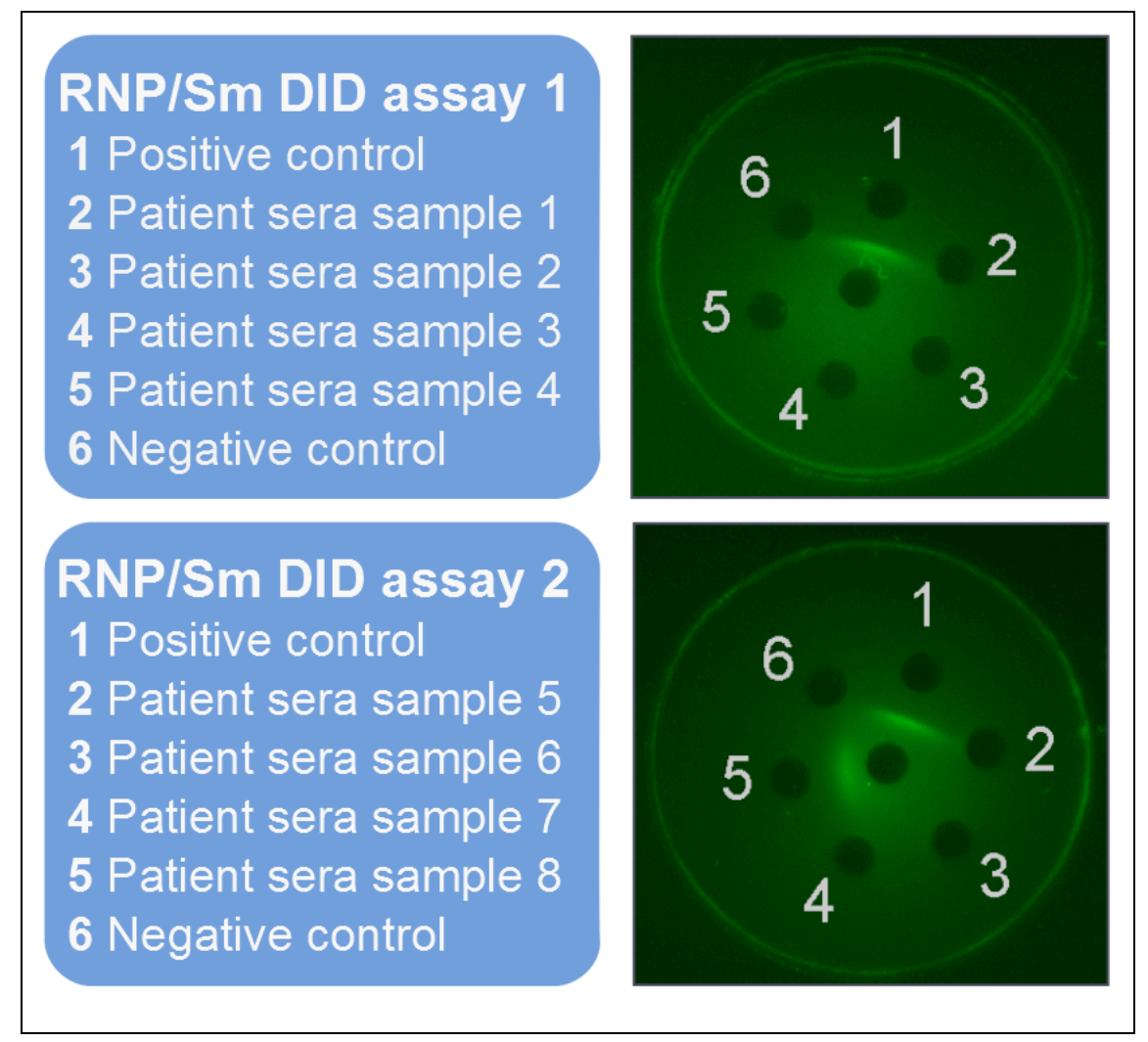

Figure $32 \mathrm{RNP} / \mathrm{Sm}$ DID assays with patient serum samples 


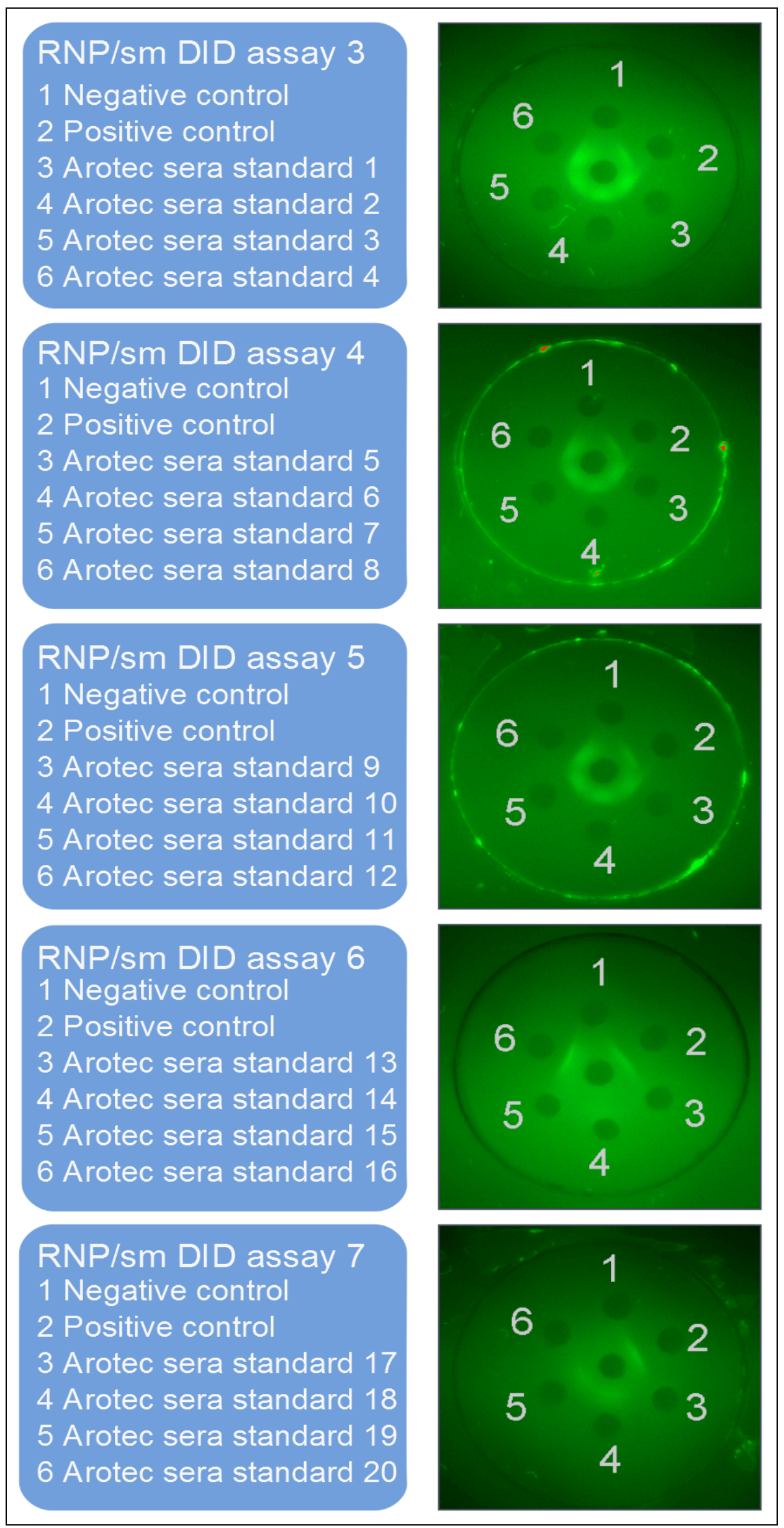

Figure 33 RNP/Sm DID assays with Arotec internal standards 
To determine the analytical sensitivity of the RNP/Sm DID assay a serum titration series was examined (Fig. 34). The point at which precipitation became indistinguishable from the negative control occurs between 1/8 and 1/16 sera dilution.

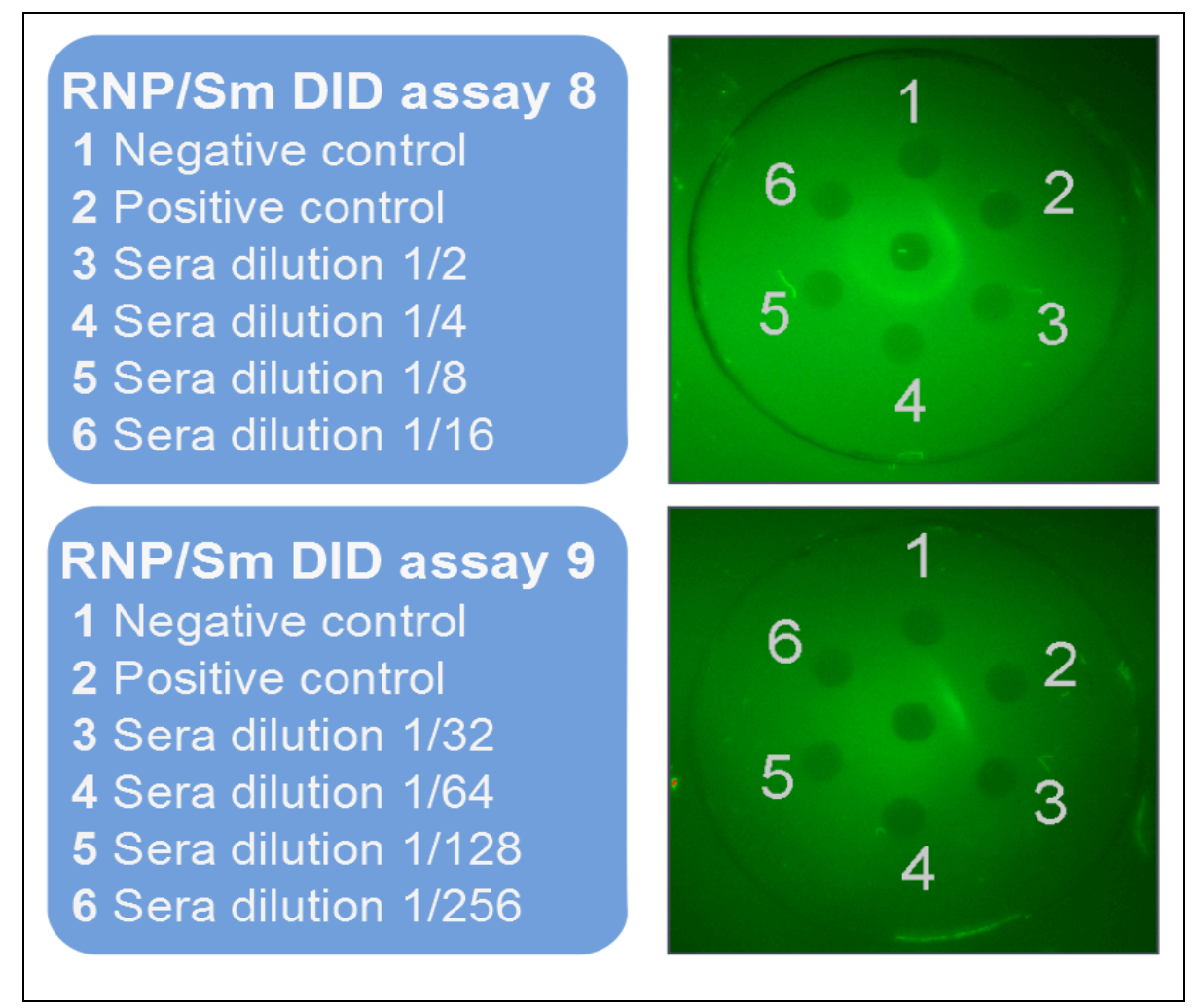

Figure 34 RNP/Sm DID assays with a serum titration series

\section{Figures 31-33 RNP/Sm DID assays}

Using an amber filter under blue light excitation the precipitin reaction was observed as a bright bands that occurred between the center well (containing the D488-labelled RNP/Sm antigen) and the numbered outer wells (containing different sera samples). 


\section{Discussion}

\subsection{Labelling and comparison of D488 vs AF488}

The overall objective of this study was to develop and validate a novel CIE assay for the detection of ANAs using fluorescent ENAs. The first objective of this study was to compare the two amine reactive dyes Alexa Fluor® 488 and Dylight 488 . To begin the method used to label antigens with D488 was examined. The complexity of the method and time required to optimally label antigens with D488 was comparable to the method and time required to label proteins with AF488.

Antigens labelled with D488 were suitable for use in diagnostic testing by CIE. A change in antibody binding caused by the labelling of an antigen with D488, would invalidate the use of said D488-labelled antigens if used for diagnostic testing by CIE. Therefore, the Western blot patterns of D488-labelled antigens (SSA, SSB, RNP/Sm, Sm, Jo-1, \& Scl-70) were examined. If labelling affected antibody binding, then as the level of D488 labelling increased, an altered pattern of antibody binding would be expected. Furthermore, this altered pattern would be exacerbated as the level of D488 bound to the antigen increased. There was no observable change in Western blot pattern when the antigens were labelled with D488. The ELISA absorbances' of unlabelled and labelled antigens (SSA, SSB, RNP/Sm, \& Sm) were also compared. These data indicate that there is no alteration of antibody binding caused by D488labelling.

The level of fluorescence produced from the D488-labelled antigens was sufficient for detection by the human eye and thus sufficient as an alternative to staining for the visualization of antigens. When the D488-labelled antigens (SSA, and RNP/Sm) are examined by SDS-PAGE and viewed by blue light excitation through an amber filter the smallest amount of protein visible by the human eye was $0.125 \mu \mathrm{g}$ for RNP/Sm, and $0.0625 \mu \mathrm{g}$ for SSA. However, when the same gels are fixed and stained with Coomassie blue the smallest amount of protein visible was $0.25 \mu \mathrm{g}$ for RNP/Sm, and $0.5 \mu \mathrm{g}$ for SSA. These results indicate that using D488-labelled proteins for CIE will not only decrease the time required for visualisation the antigen present in a gel (by 
removal of the staining step) but that visualisation of the antigen may also be improved.

D488 is superior to AF88 for the labelling of antigens. When the fluorescent intensity of D488-labelled antigens (SSA, SSB, RNP/Sm, \& Sm) was compared with the fluorescent intensity of AF488-labelled antigens the relative signal intensity produced by the D488-labelled antigens was superior to the signal intensity of the AF488labelled antigens. Also when comparing the precipitation patterns of D488-labelled and AF488-labelled antigens by CIE it was observed that D488 produced brighter bands and the observed resolution of precipitin bands using D488-labelled Sm was superior to that of AF488-labelled Sm.

\subsection{Optimisation of the ENA CIE assays}

After selection of D488 as the labelling fluor, optimisation of the CIE began with the evaluation of the agarose concentration and electric current applied during electrophoresis. These two parameters determine the speed at which the molecular components migrate through the gel and thus the overall time taken to reach assay completion. As the CIE assay was intended for use as a diagnostic tool the less time required to reach assay completion the better. Lowering the agarose concentration while inversely increasing the applied electric current decreased the time necessary to reach assay completion. However at an agarose concentration, of $0.4 \%(\mathrm{w} / \mathrm{v})$ the gel became unstable, and once the current, exceeded $5 \mathrm{~mA}$ the gel would begin to deform during the CIE. Thus the optimal conditions were determined; agarose concentration $0.6 \%(\mathrm{w} / \mathrm{v})$ and $5 \mathrm{~mA}$ applied current.

The novel CIE method contains a pre-electrophoresis step where serum is loaded onto the CIE gel and run prior to the addition of the antigen. A pre-electrophoresis step was thought to be necessary as the serum migrates relatively slowly (compared to the antigen) as its pulled along by endosmosis, and thus the position in the gel that precipitation occurs may be too close to the serum well for sufficient resolution/differentiation of a result. Pre-electrophoresis was examined and the optimal length of time for pre-electrophoresis was determined to be $5 \mathrm{~min}$. 
The buffer used for CIE is critical as it affects the electrical properties of the medium, the electrochemical properties of the molecules (serum proteins and antigens), and also influences the ability of the molecules to precipitate. It was identified in the literature that tris and tricine were suitable buffering components for CIE, and the addition of sodium-azide was also commonly added to prevent bacterial growth in the buffer/gel (Axelsen et al, 1973). As Ca-lactate was said to increase the resolution of precipitin bands, Ca-lactate was added, and its concentration optimised (Axelsen et al, 1973).. It was at this point that it was noticed that the buffer $\mathrm{pH}$ was susceptible to temperature induced changes. Three different buffers each with a different $\mathrm{pH}(8.4$, 8.6, \& 8.8 at RT) were produced (Buffer 1-3), by altering the relative ratios of Tris and Tricine. The combined concentration of Tris and Tricine were approximately the same in order to keep the conductivity approximately the same. The change in $\mathrm{pH}$ of each buffers as temperature increased was examined, and the effect of each buffer on the $\mathrm{CIE}$ assay resolution was also examined. The rate of $\mathrm{pH}$ change for each of the buffers was approximately the same and there was no observable difference in CIE assay resolution between the buffers. The temperature of a $\mathrm{CIE}$ gel during an assay run is approximately on average $30^{\circ} \mathrm{C}$ and thus Buffer $3(\mathrm{pH} 8.8$ at RT) was determined to be optimal as at this temperature it has a $\mathrm{pH}$ of 8.5 , and the optimal $\mathrm{pH}$ of a CIE assay is said to be between 8.4-8.6 (Arquembourg, 1975; Axelsen, 1973; Crowle, 1973). The optimal concentration of Tris and Tricine was determined to be 90 and $20 \mathrm{mM}$ respectively.

The optimal amount of antigen loaded during the SSA, SSB, RNP/Sm, and Sm CIE assay is $0.1 \mu \mathrm{g}(5 \mu \mathrm{l}$ at $20 \mathrm{ng} / \mu \mathrm{l})$. Less than this there is a loss of precipitin band resolution, and using antigen load greater than this provides no observable benefit in precipitin band resolution.

The analytical sensitivity for the SSA, SSB, RNP/Sm, and Sm CIE assays was examined. The limit of detection for the SSA, RNP/Sm and Sm assays occurred at a $1 / 64$ dilution in sera, and 1/4 dilution in sera for SSB. This difference in sensitivity of the SSB assay compared to the other ENA antigens was expected as a significant proportion of anti-SSB antibodies are known to be non-precipitating (Beer et al., 1996). Furthermore, anti-idiotypic antibodies present in patient serum have been shown to block binding of anti-SSB antibodies to SSB antigen peptides (Routsias et 
al., 2002). The analytical specificity of the CIE assays was examined and the ENAs were determined to be specific for the respective anti-sera.

The use of D488-labelled antigens in CIE allows for the rapid screening of patient sera for ANAs. The endpoint of the CIE assays was determined to be $30,30,50$, and 60 min for SSA, SSB, Sm, and RNP/Sm respectively. Although, for the RNP/Sm CIE assay a low fluorescent background was left in the negative control lane once the assay had reached its endpoint. This is likely due to non-specific interactions between the agarose and the RNP antigen that prevent the total migration of the antigen. This background may make differentiation between the negative control and a low titer test sample difficult. Furthermore, for the correct interpretation of result the assay run-time used must be followed stringently as some samples tested produced a clearly positive precipitin band that would migrate towards the cathodic well. If the endpoint run-time was exceeded the positive precipitin band would migrate past cathodic antigen wells producing what would appear to be a negative result (thus a false negative). See appendix 1 for further results and discussion.

\subsection{Clinical Evaluation of the ENA CIE assays}

The clinical utility of the novel anti-ENA SSA, SSB, RNP/Sm, and SM CIE assays were evaluated using a set of 281 patient serum samples sourced from the Wellington Hospital Immunology Department serum bank, and the results compared to those of an in house ELISA. Statistical analysis using Cohen's kappa statistic was performed to examine how closely the results between CIE assays and their respective ELISAs agreed with each other. The kappa statistic that tends towards 1 shows a stronger agreement and for SSA, SSB, RNP/Sm, and Sm the kappa were $0.89,0.48,0.7$, and 0.38 respectively. The strong agreement between the SSA CIE assay and ELISA indicate that one assay could be switched for another and one would get similar levels of detection. The kappa statistics for SSB and Sm indicate that there is significant difference between the CIE assay and ELISA results and that the CIE assay and ELISA could not be switched without a change in the levels of detection. Although the kappa for RNP/Sm is considered substantial it is not high enough to indicate conclusively that there is not a significant difference between the CIE assay and ELISA results. Phan et al (2001) calculated the kappa statistic for CIE vs ELISA for the following antigens SSA, SSB, and RNP/Sm. The values of which 
were $0.44,0.5$, and 0.42 for SSA, SSB, and RNP/Sm respectively. The agreement (kappa) between the novel CIE assay and ELISA for SSA, and RNP/Sm is stronger than the agreement reported by Phan et al.

Categorical clinical data was available for 156 of the 281 clinical sera samples tested. For the serum samples with categorical data available their corresponding $\mathrm{CIE}$ assay and ELISA results were grouped together into case groups. Case group 1(categorical groupings consistent with SS) consisted of samples categorised 1-4 (refer to section 2.13.3 for explanation of categories); Case group 2 (categorical grouping consistent with other CTD) consisted of samples categorised 5 and 6; Case group 3 (control group) consisted of samples in category 7 . The results of the clinical sera sample CIE assays and ELISAs in Case groups 1 and 2 were cross reference with the results in Case group 3 to determine the diagnostic specificity and sensitivity, PLR and NLR of the CIE assays and ELISAs.

When examining the performance using Group 1 data the diagnostic specificity and positive likelihood ratio (PLR) values of the SSA CIE assay were superior to those of the ELISA, while diagnostic sensitivity and the negative likelihood ratio (NLR) values were approximately the same. The sensitivity (70.8\%), specificity (90.2\%), PLR (7.24), and NLR (0.32) of the SSA CIE assay indicate that it is a highly accurate assay for the detection of SS. These results differ to those reported by Phan et al (2002) which had the sensitivity for CIE and ELISA between $85-90 \%$ and $90-97 \%$ respectively and for the specificity for CIE and ELISA between $50-60 \%$ and $45-50 \%$ respectively. Both this study and the study by Phan et al (2002) demonstrate that there are few differences between the sensitivity and specificity of CIE and ELISA. This lack of difference indicates that the high specificity of novel CIE is likely due to the sample set used to assess the CIE assay and ELISA. These observations, along with the strong inter-rater agreement between the SSA CIE assay and ELISA suggests, that the SSA CIE assay maybe a suitable replacement for ELISA as a diagnostic tool for the identification of anti-SSA antibodies and Sjogren's syndrome. Further, evaluation with a different and possibly larger sample set will be needed to assess whether the high specificity of the novel CIE assay is due to the sample set used to evaluate or to the inherent methodology of the technique. 
Group 1 data was used to compare the RNP/Sm CIE assay and ELISA. The statistical measures of assay accuracy (diagnostic specificity and sensitivity, PLR, and NLR) for the RNP/Sm CIE assay and ELISA were approximately the same. However the values of the statistics for the ELISA were superior to those of CIE assay. Thus there is little evidence for clinical utility for this RNP/Sm CIE assay.

Examining Group 1 data, the diagnostic specificities for the SSB CIE assay (98.9\%) and Sm (97.8\%) CIE assay were higher than that of their respective ELISAs $(89.1 \%$, and $85.9 \%$ respectively). The diagnostic sensitivities for the SSB CIE assay (16.7\%) and $\mathrm{Sm}(12.5 \%)$ CIE assay were much lower than that of their respective ELISAs (54.2\%, and 33.3\% respectively). The PLR of the SSB CIE assay (15.33) was approximately 3 times that of its respective ELISA (4.98), and the PLR of the Sm CIE assay (5.75) was approximately twice that of its respective ELISA (2.36). Compared to Phan et al (2002) the sensitivity for CIE and ELISA for SSB was between $70-80 \%$ and $75-85 \%$ respectively and for the specificity for CIE and ELISA between $60-70 \%$ and $50-60 \%$ respectively. These results indicate that for the SSB and Sm CIE assays there would be a low occurrence of false positives and that these assays would be suitable as secondary confirmatory diagnostic assays for the identification of their respective ENA antibodies. Again, the results from Phan et al (2002) show few differences between the sensitivity and specificity of CIE and ELISA. In terms of the specificity determinied in this study this is also true. However there is a clear difference in sensitivity between the CIE and ELISA that is not seen in the study Phan by et al. This difference, along with the low agreement between the two assays indicates that there is some difference between the novel CIE assay and the ELISA that is likely not simply due to the sample set used to evaluate them. There was no reported data for the diagnostic sensitivity and specificity for SS as relates to Sm, as $\mathrm{Sm}$ is not considered a marker for SS. Again these results emphasise the need to further evaluate the novel CIE assays.

When examining the CIE assays and ELISAs that using Case group 2 data does not change the diagnostic specificities for all assays the values for Case group 3 (the control group) have not changed. The sensitivity for all assays is low (less than $22 \%$ ) and the values of the PLR and NLR ratio for all CIE and ELISA assays in Case group 2 tend towards 1 . Thus from the predictive value of either the CIE assays or the 
ELISAs for other connective tissue diseases cannot be inferred. This may be due to the sample set tested being to small and/or the clinical data provided which essentially groups patients into other connective tissue disease. A clinical data set that indicated specifically what other connective tissue disease the patient symptoms indicated would be preferred to the current clinical data. Better yet would be to assess the diagnostic value of the assays by performing a longitudinal study in a clinical laboratory.

\subsection{RNP/Sm DID assay}

As it appears that the novel RNP/Sm CIE assay may not be suitable for use as a diagnostic tool, an alternative use for the D488-labelled RNP/Sm antigen was examined in the form of a DID assay. A series of experiment were performed to determine if there was a possibility of using D488-labelled RNP/Sm antigen for DID. For the 8 clinical samples and 20 Arotec internal standards tested those sera which were anti-RNP/Sm positive produced positive results by DID and those which were anti-RNP/Sm negative produced negative results by DID. Also the analytical sensitivity of this RNP/Sm DID assay was examined. A 1/8 sera dilution was the limit of detection and is notably less sensitive than that of the CIE assay. Promisingly these results indicate that a DID assay using D488-labelled RNP/Sm antigen could be used for the detection of anti-RNP/Sm serum antibodies. 


\section{Conclusions and Future Work}

The central aim of this project to develop and validate a novel counter immunoelectrophoresis (CIE) assay that uses fluorescently labelled ENAs to detect ENA antibodies in patient serum was achieved for the SSA, SSB, and Sm antigens.

Optimisation of CIE assay conditions for the detection ANA to each of the D488labelled ENAs SSA, SSB, RNP/Sm, and Sm was successful. Assay conditions optimised were flurophore type, gel composition, buffer composition, antigen concentration, the running time, and analytical specificity. The CIE assays were highly specific, and their accuracy was comparable if not superior to that of ELISA.

The SSA CIE assay results suggest that this assay maybe a suitable replacement for ELISA as a diagnostic tool for the identification of anti-SSA antibodies and Sjogren's syndrome. The SSB and Sm CIE assay results suggest that these assays would be suitable as secondary confirmatory diagnostic assays for the identification of their respective ENA antibodies. Although, results are promising further performance evaluation of the assays is necessary. This would require assessment of the assays as part of a longitudinal study within a routine diagnostic laboratory where patient outcome is tracked. A study of this type is required to provide a more accurate clinical data set for the assessment of the assays accuracy and to then compare these results alongside the laboratory's current diagnostic testing methods.

The D488-labelled RNP/Sm CIE assay was not suitable for use as a diagnostic tool. This was because it results were less accurate than ELISA, and it produced a low background in the negative control which made it difficult to objectively interpret the results of low titer plasma samples. However, the D488-labelled RNP/Sm may make a suitable antigen for DID as the preliminary results suggest. Further evaluation of the RNP/Sm DID assay with a greater number of samples is required for statistical analysis of its predictive accuracy. 


\section{References}

Anhalt, J. P., \& Yu, P. K. (1975). Counterimmunoelectrophoresis of pneumococcal antigens: improved sensitivity for the detection of types VII and XIV. J Clin Microbiol, 2(6), 510-515.

Arquembourg, P. C. (1975). Immunoelectrophoresis; Theory, Methods, Identification, Interpretation (2nd ed.). Switzerland: Tanner \& Bosshardt AG.

Axelsen, N. H., Kroll, J., Weeke, B. (Ed.). (1973). A Manual of Quantitative Immunoelectrophoresis; Methods and Applications. Norway: Blackwell Scientific Publications.

Beer, R. G., Rischmueller, M., Coates, T., Purcell, A. W., Keech, C. L., McCluskey, J., \& Gordon, T. P. (1996). Nonprecipitating anti-La(SS-B) autoantibodies in primary Sjogren's syndrome. Clin Immunol Immunopathol, 79(3), 314-318.

Bernstein, R. M., Morgan, S. H., Chapman, J., Bunn, C. C., Mathews, M. B., TurnerWarwick, M., \& Hughes, G. R. (1984). Anti-Jo-1 antibody: a marker for myositis with interstitial lung disease. Br Med J (Clin Res Ed), 289(6438), 151-152.

Bhattacharya, S., \& Sinha, J. K. (2006). A Textbook of Immunology. India: Academic Publishing.

Colglazier, C. L., \& Sutej, P. G. (2005). Laboratory testing in the rheumatic diseases: a practical review. South Med J, 98(2), 185-191.

Crowle, A. J. (1973). Immunodiffusion (2 ed.). New York: Academic Press.

Dahle, C., Skogh, T., Aberg, A. K., Jalal, A., \& Olcen, P. (2004). Methods of choice for diagnostic antinuclear antibody (ANA) screening: benefit of adding antigen-specific assays to immunofluorescence microscopy. J Autoimmun, 22(3), 241-248.

Deeks, J. J., \& Altman, D. G. (2004). Diagnostic tests 4: likelihood ratios. BMJ, 329(7458), 168-169.

Defendenti, C., Atzeni, F., Spina, M. F., Grosso, S., Cereda, A., Guercilena, G., . . Puttini, P. S. (2011). Clinical and laboratory aspects of Ro/SSA-52 autoantibodies. Autoimmun Rev, 10(3), 150-154.

Edelman, G. M., Gall, W. E., Waxdal, M. J., \& Konigsberg, W. H. (1968). The covalent structure of a human gamma G-immunoglobulin I. Isolation and characterization of the whole molecule, the polypeptide chains, and the tryptic fragments. Biochemistry, 7(5), 1950-1958. 
Elgert, K. D. (1996). Immunology: Understanding the Immune System. New York: WileyLiss.

Franceschini, F., \& Cavazzana, I. (2005). Anti-Ro/SSA and La/SSB antibodies. Autoimmunity, 38(1), 55-63.

Frey, A., Di Canzio, J., \& Zurakowski, D. (1998). A statistically defined endpoint titer determination method for immunoassays.[Research Support, Non-U.S. Gov't].J Immunol Methods, 221(1-2), 35-41.

Gomez-Puerta, J. A., Burlingame, R. W., \& Cervera, R. (2008). Anti-chromatin (antinucleosome) antibodies: diagnostic and clinical value. Autoimmun Rev, 7(8), 606611.

Goodnow, C. C., Sprent, J., Fazekas de St Groth, B., \& Vinuesa, C. G. (2005). Cellular and genetic mechanisms of self tolerance and autoimmunity. Nature, 435(7042), 590597.doi: $10.1038 /$ nature03724

Goodnow, C. C., Vinuesa, C. G., Randall, K. L., Mackay, F., \& Brink, R. (2010). Control systems and decision making for antibody production. Immunol, 11(8), 681-688.

Hargraves, M. M., Richmond, H., \& Morton, R. (1948). Presentation of two bone marrow elements; the tart cell and the L.E. cell. Proc Staff Meet Mayo Clin, 23(2), 25-28.

Haserick, J. R., \& Bortz, D. W. (1949). Normal bone marrow inclusion phenomena induced by lupus erythematosus plasma. J Invest Dermatol, 13(2), 47-49.

Hepburn, A. L. (2001). The LE cell. Rheumatology (Oxford), 40(7), 826-827.

Hernandez-Molina, G., Leal-Alegre, G., \& Michel-Peregrina, M. (2011). The meaning of anti-Ro and anti-La antibodies in primary Sjogren's syndrome. Autoimmun Rev, 10(3), 123-125.

Keren, D. F. (2002). Antinuclear antibody testing. Clin Lab Med, 22(2), 447-474.

Kerr, M. A. (2006). In the blood: the structure of immunoglobulin. Biochemical Journal.

Klein, L., Hinterberger, M., Wirnsberger, G., \& Kyewski, B. (2009). Antigen presentation in the thymus for positive selection and central tolerance induction Nat Rev Immunol, 9(12), 833-844.

Kraemer, H. C. (1980). Extension of the kappa coefficient. Biometrics, 36(2), 207-216.

Kveder, T., \& Bozic, B. (2002). Counterimmunoelectrophoresis: fast, easy and costeffective method for the detection of autoantibodies to intracellular antigens. Clin Chem Lab Med, 40(4), 428-429.

Landis, J. R., \& Koch, G. G. (1977). The measurement of observer agreement for categorical data. Biometrics, 33(1), 159-174. 
Lleo, A., Invernizzi, P., Gao, B., Podda, M., \& Gershwin, M. E. (2010). Definition of human autoimmunity--autoantibodies versus autoimmune disease. Autoimmun Rev, 9(5), 259-266.

Manoussakis, M. N., Kistis, C. G., Aidinis, V., Guialis, A., Piha, L., Sekeris, C. E., \& Moutsopoulos, H. M. (1995). Detection of human-specific anti-la(SSB) antibodies in patients with rheumatoid arthritis. J Autoimmun, 8(6), 959-969.

Manoussakis, M. N., Kistis, K. G., Liu, X., Aidinis, V., Guialis, A., \& Moutsopoulos, H. M. (1993). Detection of anti-Ro(SSA) antibodies in autoimmune diseases: comparison of five methods. Br J Rheumatol, 32(6), 449-455.

McHeyzer-Williams, L. J., \& McHeyzer-Williams, M. G. (2005). Antigen-specific memory B cell development. Annu Rev Immunol, 23, 487-513.

Meilof, J. F., Bantjes, I., De Jong, J., Van Dam, A. P., \& Smeenk, R. J. (1990). The detection of anti-Ro/SS-A and anti-La/SS-B antibodies.A comparison of counterimmunoelectrophoresis with immunoblot, ELISA, and RNA-precipitation assays. J Immunol Methods, 133(2), 215-226.

Melamed, D., Benschop, R. J., Cambier, J. C., \& Nemazee, D. (1998). Developmental regulation of $B$ lymphocyte immune tolerance compartmentalizes clonal selection from receptor selection. Cell, 92(2), 173-182.

Migliorini, P., Baldini, C., Rocchi, V., \& Bombardieri, S. (2005). Anti-Sm and anti-RNP antibodies. Autoimmunity, 38(1), 47-54.

Mills, K. H. (2004). Regulatory T cells: friend or foe in immunity to infection?. Nat Rev Immunol, 4(11), 841-855.

Mills, K. H. (2011). TLR-dependent T cell activation in autoimmunity. Nat Rev Immunol, 11(12), 807-822.

Nardi, N., Brito-Zeron, P., Ramos-Casals, M., Aguilo, S., Cervera, R., Ingelmo, M., \& Font, J. (2006). Circulating auto-antibodies against nuclear and non-nuclear antigens in primary Sjogren's syndrome: prevalence and clinical significance in 335 patients. Clin Rheumatol, 25(3), 341-346.

Paul, W. E. (2013). Fundamental immunology (7th ed.). Philadelphia: Wolters Kluwer Health/Lippincott Williams \& Wilkins.

Phan, T. G., Ng, W. W., Bird, D., Smithers, K., Wong, V., Gallagher, K., \& Adelstein, S. (2001). High-quality, cost-effective strategy for detection of autoantibodies to extractable nuclear antigens. Clin Diagn Lab Immunol, 8(3), 471-474. 
Phan, T. G., Wong, R. C., \& Adelstein, S. (2002). Autoantibodies to extractable nuclear antigens: making detection and interpretation more meaningful. Clin Diagn Lab Immunol, 9(1), 1-7.

Porter, R. R. (1959). The hydrolysis of rabbit $Y$-globulin and antibodies with crystalline papain.Biochem J, 73, 119-126.

Reichlin, M., \& Harley, J. B. (1986). Detection by ELISA of antibodies to small RNA protein particles in systemic lupus erythematosus patients whose sera lack precipitins. Trans Assoc Am Physicians, 99, 161-171.

Roncarolo, M. G., \& Battaglia, M. (2007). Regulatory T-cell immunotherapy for tolerance to self antigens and alloantigens in humans. Nat Rev Immunol, 7(8), 585-598.

Roosnek, E., \& Lanzavecchia, A. (1991). Efficient and selective presentation of antigenantibody complexes by rheumatoid factor B cells. J Exp Med, 173(2), 487-489.

Routsias, J. G., Touloupi, E., Dotsika, E., Moulia, A., Tsikaris, V., Sakarellos, C., \& Tzioufas, A. G. (2002). Unmasking the anti-La/SSB response in sera from patients with Sjogren's syndrome by specific blocking of anti-idiotypic antibodies to La/SSB antigenic determinants. Mol Med, 8(6), 293-305.

Selmi, C. (2011). Autoimmunity in 2010. Autoimmun Rev, 10(12), 725-732.

Sheldon, J. (2004). Laboratory testing in autoimmune rheumatic diseases. Best Pract Res Clin Rheumatol, 18(3), 249-269.

Shmerling, R. H. (2005). Diagnostic tests for rheumatic disease: clinical utility revisited. South Med J, 98(7), 704-711.

Shoenfeld, Y., \& Tincani, A. (2005). Autoantibodies-the smoke and the fire. Autoimmunity, $38(1), 1-2$.

Solomon, D. H., Kavanaugh, A. J., \& Schur, P. H. (2002). Evidence-based guidelines for the use of immunologic tests: antinuclear antibody testing. Arthritis Rheum, 47(4), 434-444.

Tonuttia, E., Bassetti, D., Piazza, A., Visentini, D., Poletto, M., Bassetto, F., Caciagil, P., Villalta, D., Tozzoli, R., \& Bizzaro, N. (2004). Diagnostic accuracy of ELISA methods as an alternative screening test to indirect immunofluorescence for the detection of antinuclear antibodies. Evaluation of five commercial kits. Autoimmunity, 37(2), 171176.

Vaman Rao, C. (2006). Immunology (second ed.). Oxford, U.K. Alpha Science International Ltd. 
Verbruggen. (1975). Quantitative immunoelectrophoretic methods: a literature survey. Clin Chem, 21(1), 5-43.

Westermeier, R. (2001). Electrophoresis in Practice (3rd ed.). Weinheim: WILEY-VCH. 


\section{Appendix 1}

\section{Experiment $\mathbf{A} 1$}

Aim To examine what effect of serum age has on the outcome of a CIE assay replicate experiments were performed using a sera dilution series

Method Three 2-fold sera dilution series (range 1/2 to 1/256) were created using Sm positive sera and unreactive sera (negative control). Using the dilution series and D488labelled Sm antigen at a concentration of $20 \mathrm{ng} / \mu \mathrm{l}$ a CIE assay was performed. The dilution series was stored at $4^{\circ} \mathrm{C}$ for 4 months and the CIE assay repeated. All CIE assays were performed using Apparatus 3 and the standard CIE method.

Results Examining the precipitin bands in lane 5 (Fig. A1): when the serum was first run (0 months) at the endpoint of the assay (50 min runtime) a strong precipitin band half way between the $\mathrm{Ag}$ and $\mathrm{Ab}$ wells was observed. When the sera was run again (4 months) at the endpoint (50 min runtime) of the assay, the precipitin band was located slightly below the Ag well. If the CIE assay is run for a further 5 min the precipitin band migrates towards the cathode and past the Ag wells.

Discussion These results suggest that as sera ages it produces a weaker more diffuse precipitate that can also migrate towards the cathode (towards the Ag well). This is likely due to the breakdown of either the serum antibodies and/or the serum proteins that assist precipitate formation. Notably, this effect seems to decrease as the titer of the sera decreases. This suggests that breakdown products are interacting and blocking the antigen interaction sites. Thus in antigen excess there are an increased number of unblocked interaction sites and therefore precipitate formation is restored.

It is also worth noting that when the assay was run for a period longer than 50 min the precipitin band ran past the $\mathrm{Ag}$ well in lane 5 giving the impression of a negative result. This highlights the importance of having a stringent assay runtime. 


\section{Sm sera titrations at 0 and 4 months}

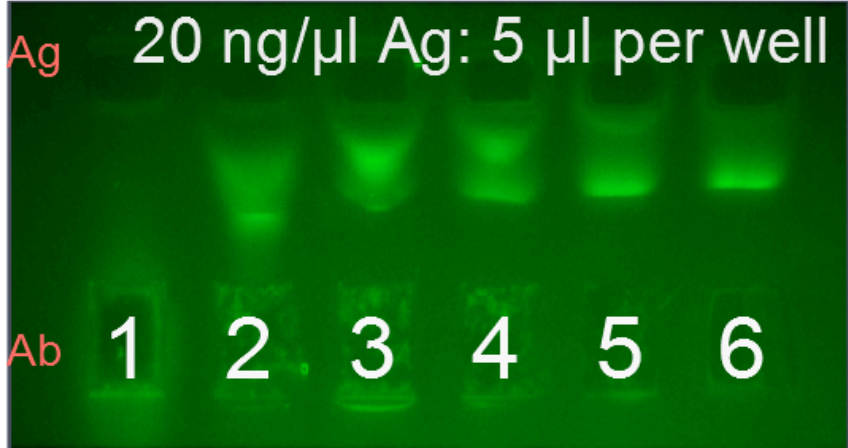

Sera age: 0 months Runtime: 50 min

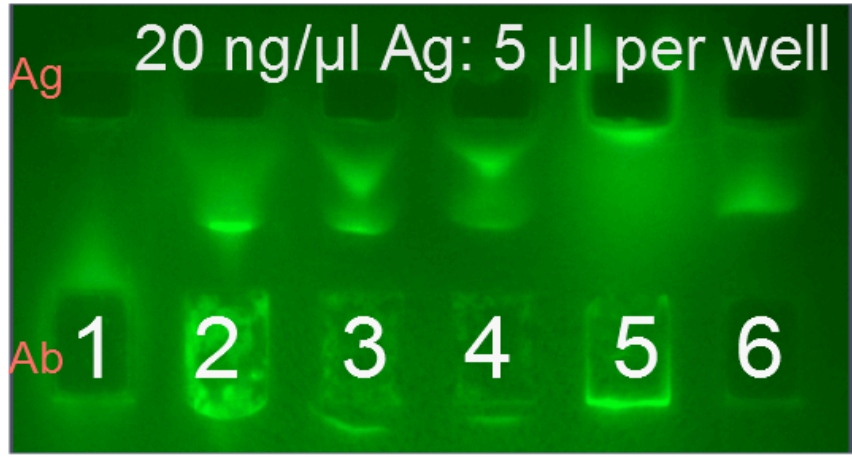

Sera age: 4 months Runtime: 50 min

Ag $20 \mathrm{ng} / \mu \mathrm{l} \mathrm{Ag:} 5 \mu \mathrm{l}$ per well

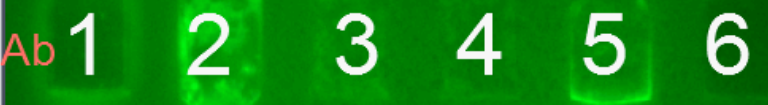

Sera age: 4 months Runtime: 55 min

Figure A1 Sm sera titrations at 0 and 4 months

\section{Figure A1}

Replicate CIE assays performed with the same sera dilution series 4 months apart. In the Ag wells $5 \mu$ l antigen was loaded into each well. A sera titration series was loaded into the Ab wells; Lane 1 negative control, Lanes 2-5 2-fold sera dilutions $1 / 16$ to $1 / 2$, Lane 6 positive control. Using an amber filter under blue light excitation the precipitin reaction was observed as bright horizontal bands. 